NBER WORKING PAPER SERIES

\title{
EFFICIENCY OF FLEXIBLE BUDGETARY INSTITUTIONS
}

\author{
T. Renee Bowen \\ Ying Chen \\ Hülya K. Eraslan \\ Jan Zápal \\ Working Paper 22457 \\ http://www.nber.org/papers/w22457
NATIONAL BUREAU OF ECONOMIC RESEARCH
1050 Massachusetts Avenue
Cambridge, MA 02138
July 2016

We thank discussants Marina Azzimonti and Antoine Loeper. We also thank Gabriel Carroll, Sebastien DiTella, Roger Lagunoff, Alessandro Riboni and seminar and conference participants at Stanford University, Universitat Autonoma de Barcelona, Duke University, Ural Federal University, University of Chicago, University of Mannheim, University of Warwick, LSE, University of Nottingham, UC Berkeley, Max Planck Institute in Bonn, Paris Workshop in Political Economy, the NBER Summer Institute Political Economy and Public Finance Workshop, the 2014 SITE Workshop on the Dynamics of Collective Decision Making, SED 2015 in Warsaw, SAET 2015 in Cambridge, EEA 2015 in Mannheim, and Econometric Society 2015 World Congress in Montreal for helpful comments and suggestions. The views expressed herein are those of the authors and do not necessarily reflect the views of the National Bureau of Economic Research.

NBER working papers are circulated for discussion and comment purposes. They have not been peer-reviewed or been subject to the review by the NBER Board of Directors that accompanies official NBER publications.

(C) 2016 by T. Renee Bowen, Ying Chen, Hülya K. Eraslan, and Jan Zápal. All rights reserved. Short sections of text, not to exceed two paragraphs, may be quoted without explicit permission provided that full credit, including $(\mathrm{C}$ notice, is given to the source. 
Efficiency of Flexible Budgetary Institutions

T. Renee Bowen, Ying Chen, Hülya K. Eraslan, and Jan Zápal

NBER Working Paper No. 22457

July 2016

JEL No. C73,C78,D61,D78,H61

\begin{abstract}
$\underline{\text { ABSTRACT }}$
Which budgetary institutions result in efficient provision of public goods? We analyze a model with two parties bargaining over the allocation to a public good each period. Parties place different values on the public good, and these values may change over time. We focus on budgetary institutions that determine the rules governing feasible allocations to mandatory and discretionary spending programs. Mandatory spending is enacted by law and remains in effect until changed, and thus induces an endogenous status quo, whereas discretionary spending is a periodic appropriation that is not allocated if no new agreement is reached. We show that discretionary only and mandatory only institutions typically lead to dynamic inefficiency and that mandatory only institutions can even lead to static inefficiency. By introducing appropriate flexibility in mandatory programs, we obtain static and dynamic efficiency. An endogenous choice of mandatory and discretionary programs, sunset provisions and state-contingent mandatory programs can provide this flexibility in increasingly complex environments.
\end{abstract}

\author{
T. Renee Bowen \\ Graduate School of Business \\ Stanford University \\ 655 Knight Way \\ Stanford, CA 94305 \\ and NBER \\ trbowen@stanford.edu \\ Ying Chen \\ Johns Hopkins University \\ Department of Economics \\ Wyman Park Building 544E \\ 3400 N Charles St \\ Baltimore, MD 21218 \\ ying.chen@jhu.edu
}

\author{
Hülya K. Eraslan \\ Department of Economics MS-22 \\ Rice University \\ P.O. Box 1892 \\ Houston, TX 77251-1892 \\ eraslan@rice.edu \\ Jan Zápal \\ CERGE-EI \\ Politickych veznu 7 \\ Prague, 11121 \\ Czech Republic \\ and IAE-CSIC and Barcelona GSE \\ j.zapal@cerge-ei.cz
}




\section{Introduction}

Allocation of resources to public goods is typically decided through budget negotiations. In many democratic governments these negotiations occur annually and are constrained by the budgetary institutions in place. In designing budgetary institutions one may have various goals, such as efficiency, responsiveness to citizens' preferences, or accountability. There has been increasing interest among policy-makers in understanding how to achieve these goals in both developed and developing countries (see, for example, Santiso, 2006; Shah, 2007). ${ }^{1}$ Economic research has also recognized the importance of budgetary institutions (see, for example, Hallerberg, Strauch and von Hagen, 2009). ${ }^{2}$ These studies emphasize the importance of various dimensions of budgetary institutions including transparency and centralization of decision-making. We focus on a different dimension in this paper: the rules governing feasible allocations to mandatory and discretionary spending programs. ${ }^{3}$ Discretionary programs require periodic appropriations, and no spending is allocated if no new agreement is reached. By contrast, mandatory programs are enacted by law, and spending continues into the future until changed. Thus under mandatory programs, spending decisions today determine the status quo level of spending for tomorrow.

Naturally, there may be disagreement on the appropriate level of public spending, and the final spending outcome is the result of negotiations between parties that represent different interests. Negotiations are typically led by the party in power whose identity may change over time, bringing about turnover in agenda-setting power. Bowen, Chen and Eraslan (2014) show that in a stable economic environment, mandatory programs improve the efficiency of public good provision over discretionary programs by mitigating the inefficiency due to turnover. However, the economic environment may be changing over time, potentially re-

\footnotetext{
${ }^{1}$ The OECD has devoted resources to surveying budget practices and procedures across countries since 2003. See International Budget Practices and Procedures Database, OECD (2012).

${ }^{2}$ See also Alesina and Perotti (1995) for a survey of the early literature recognizing the importance of budgetary institutions.

${ }^{3}$ This terminology is used in the United States budget. Related institutions exist in other budget negotiations, for example the budget of the European Union is categorized into commitment and payment appropriations. The main distinction is that one has dynamic consequences because agreements are made for future budgets, and the other does not.
} 
sulting in evolving preferences. Hence, the party in power today must consider how current spending on the public good affects future spending when preferences and the agenda-setter are possibly different from today. In this paper we investigate the role of budgetary institutions in the efficient provision of public goods in an environment with these features. Specifically, we analyze an environment with disagreement over the value of the public good, changing economic conditions, and turnover in political power.

In this environment it is natural to expect that mandatory programs may have some drawbacks. Indeed, in settings different from ours, Riboni and Ruge-Murcia (2008), Zapal (2011) and Dziuda and Loeper (2015) note that inefficiency can arise from mandatory programs when preferences are evolving. ${ }^{4}$ In accord with these results, we first show that mandatory programs in isolation lead to inefficiency in public good spending, but the main contribution of our paper is to show that efficiency can be obtained when appropriate flexibility is added to mandatory programs. We show this in increasingly complex environments.

We begin by analyzing a model in which two parties bargain over the spending on a public good in each of two periods. The parties place different values on the public good, and these values may deterministically change over time, reflecting changes in the underlying economic environment. To capture turnover in political power, we assume the proposing party is selected at random each period. Unanimity is required to implement the proposed spending on the public good. We investigate the efficiency properties of the equilibrium outcome of this bargaining game under different budgetary institutions.

We distinguish between static Pareto efficiency and dynamic Pareto efficiency. A statically Pareto efficient allocation in a given period is a spending level such that no alternative would make both parties better off and at least one of them strictly better off in that period. A dynamically Pareto efficient allocation is a sequence of spending levels, one for each period, that needs to satisfy a similar requirement except that the utility possibility frontier is constructed using the discounted sum of utilities. Dynamic efficiency puts intertemporal restrictions on spending levels in addition to requiring static efficiency for each period, making it a stronger requirement than static Pareto efficiency. We show that if parties disagree

\footnotetext{
${ }^{4}$ We further discuss how our results relate to these and other papers at the end of the Introduction.
} 
about the value of the public good in all periods, then any equilibrium in which spending varies with the identity of the proposer cannot be dynamically Pareto efficient. That is, dynamic Pareto efficiency requires that parties insure against political risk. We further show that when preferences are evolving over time, dynamic Pareto efficiency typically requires that spending levels change accordingly. This means that with evolving preferences dynamic Pareto efficiency requires that parties avoid gridlock.

Comparing equilibrium public good allocations with the efficient ones, we show that discretionary only institutions lead to static efficiency but dynamic inefficiency, mandatory only institutions can lead to static and dynamic inefficiency, whereas allowing an endogenous combination of mandatory and discretionary programs results in both static and dynamic efficiency if the value of the public good is decreasing over time. Furthermore, if temporary cuts to mandatory programs are allowed, an endogenous choice of mandatory and discretionary programs results in both static and dynamic efficiency for any deterministic change in the value of the public good. Sequestration and furloughs are examples of temporary cuts to mandatory programs seen in practice.

The primary reason for dynamic inefficiency of discretionary only budget institutions is that they lead to political risk. Specifically, since the status quo of a discretionary spending program is exogenously zero, the equilibrium level of spending varies with the party in power.

With mandatory only budgetary institutions, any equilibrium is dynamically inefficient because the second period's spending level either varies with the identity of the proposing party, which leads to political risk, or is equal to the first period's level, which results in gridlock. Even static inefficiency may result with mandatory only budget institutions. This is because the parties' concerns about their future bargaining positions, which are determined by the first period's spending level, can lead the parties to reach an outcome that goes against their first-period interests.

In contrast, budgetary institutions that allow flexibility with a combination of discretionary and mandatory programs avoid both political risk and gridlock, resulting in dynamic efficiency. ${ }^{5}$ To see why this is true, first consider the case when the value of the public good is

\footnotetext{
${ }^{5}$ Examples of budget functions in the United States with significant fractions of both mandatory and
} 
decreasing over time for both parties. The party in power in the first period finds it optimal to set the size of the mandatory program to a level that is statically efficient in the second period. Given this, the status quo is maintained in the second period regardless of which party comes into power, thereby eliminating political risk. The party in power in the first period can then use discretionary spending to increase the total spending to the desired level in the first period, avoiding gridlock. Thus dynamic efficiency is achieved by a combination of positive discretionary and mandatory spending. Now consider any time profile of public good spending (either increasing or decreasing) that dynamic efficiency might require. If discretionary spending can be negative, which we interpret as a temporary cut in mandatory programs, then the party in power in the first period can tailor the spending to the desired level and achieve dynamic efficiency. The main insight is that the flexibility afforded by a combination of mandatory and discretionary programs delivers efficiency. However, this efficiency result breaks down with a longer time horizon because to eliminate political risk in all future periods, the first-period proposer must be able to set all future status quos independently, which is not feasible with a simple combination of mandatory and discretionary programs. In this case, we show that efficiency is achieved with sunset provisions with appropriately chosen expiration dates.

To extend our result to an even richer environment, we consider a model with an arbitrary time horizon and stochastic preferences that depend on the economic state. We analyze a budgetary institution in which proposers choose a spending rule that gives spending levels conditional on the realization of the state. We show that the first-period proposer chooses a rule that is dynamically efficient and once chosen, this spending rule is retained because no future proposer can make a different proposal that is better for itself and acceptable to the other party. Thus state-contingent mandatory programs allow sufficient flexibility to achieve dynamic efficiency, even though we consider spending rules that cannot condition on the proposer identity.

discretionary spending include income security, commerce and housing credit, and transportation (see Budget of the United States Government, 2015). Policymakers explicitly specify the budget enforcement act category, that is, mandatory or discretionary, when proposing changes to spending on budget functions (see, for example, House Budget Committee, 2014). 
The use of state-contingent programs dates back to at least Ancient Egypt, where the rate of taxation depended on the extent of Nile flooding in any given year (see Breasted, 1945, page 191). Such state-contingency can also be found in practice in modern economies as automatic adjustments embedded in mandatory programs. For example, in the United States unemployment insurance may fluctuate with the unemployment rate through "extended" or "emergency" benefits. These benefits have been a feature of the unemployment insurance law since 1971, and are triggered by recession on the basis of certain unemployment indicators (see Nicholson and Needels, 2006). ${ }^{6}$ Similarly, in Canada the maximum number of weeks one can receive unemployment benefits depends on the local rate of unemployment (see Canadian Minister of Justice, 2014, Schedule I, page 180). The efficiency of state-contingent spending programs may explain why they are successfully implemented in practice.

Our work is related to several strands of literature. A large body of political economy research studies efficiency implications of policies that arise in a political equilibrium. ${ }^{7}$ As highlighted in Besley and Coate (1998) inefficiency can arise because policies either yield benefits in the future when the current political representation might not enjoy them, or alter the choices of future policy makers, or may change the probability of the current political representation staying in power. Our paper shares with the rest of the literature the first two sources of inefficiency, but unlike the rest of the literature, our main focus is on linking these sources of inefficiency to budgetary institutions that specify the rules governing feasible allocations to mandatory and discretionary spending programs.

Modeling mandatory spending programs as an endogenous status quo links our work to a growing dynamic bargaining literature. ${ }^{8}$ With the exception of Bowen, Chen and Eraslan

\footnotetext{
${ }^{6}$ See also Federal-State Extended Unemployment Compensation Act of 1970, U.S. House of Representatives, Office of the Legislative Counsel (2013).

${ }^{7}$ See, for example, Persson and Svensson (1984); Alesina and Tabellini (1990); Krusell and Ríos-Rull (1996); Dixit, Grossman and Gul (2000); Lizzeri and Persico (2001); Battaglini and Coate (2007); Acemoglu, Golosov and Tsyvinski (2008, 2011); Aguiar and Amador (2011); Azzimonti (2011); Bai and Lagunoff (2011); Van Weelden (2013); Callander and Krehbiel (2014); Bierbrauer and Boyer (2014).

${ }^{8}$ This literature includes Baron (1996); Kalandrakis (2004, 2010); Riboni and Ruge-Murcia (2008); Diermeier and Fong (2011); Zapal (2011); Battaglini and Palfrey (2012); Duggan and Kalandrakis (2012); Piguillem and Riboni (2012, 2015); Diermeier, Egorov and Sonin (2013); Levy and Razin (2013); Baron and Bowen (2014); Bowen, Chen and Eraslan (2014); Chen and Eraslan (2014); Forand (2014); Kalandrakis (2014); Ma (2014); Nunnari and Zapal (2014); Dziuda and Loeper (2015); Anesi and Seidmann (2015).
} 
(2014) and Zapal (2011) this literature has focused on studying models only with policies that have the endogenous status quo property. In the language of our model, this literature has focused on mandatory spending programs only. Bowen, Chen and Eraslan (2014) model discretionary and mandatory spending programs, but do not allow for an endogenous choice of these two types of programs. Moreover, unlike in their model, we allow the values parties attach to the public good to vary over time, which plays an important role in our results. Bowen, Chen and Eraslan (2014) show that mandatory programs ex-ante Pareto dominate discretionary programs under certain conditions, whereas we show that with evolving preferences mandatory programs with appropriate flexibility achieve dynamic efficiency. Zapal (2011) demonstrates that a budgetary institution that allows for distinct current-period policy and future-period status quo eliminates static inefficiency. This result parallels the efficiency of an endogenous choice of mandatory and discretionary programs that we show, but we do this in an environment with political turnover and more general variation in preferences. Furthermore, we also demonstrate the efficiency of state-contingent mandatory programs in this richer setting.

Our focus on budgetary institutions connects our work to papers studying fiscal rules and fiscal constitutions. ${ }^{9}$ This literature has focused on other fiscal rules or constitutions, for example, constraints on government spending and taxation, limits on public debt or deficits, or decentralization of spending authority.

In the next section we describe our model. In Section 3 we discuss Pareto efficient allocations and define Pareto efficient equilibria. We discuss institutions with only discretionary spending in Section 4. In Section 5 we give properties of equilibria for institutions that allow mandatory spending (with or without discretionary spending), and give efficiency properties of mandatory only institutions. In Section 6 we discuss institutions that allow for an endogenous choice of mandatory and discretionary spending, as well as sunset provisions. In Section 7 we consider state-contingent mandatory spending. We conclude in Section 8. All proofs omitted in the main text are in the Appendix.

\footnotetext{
${ }^{9}$ See, for example, Persson and Tabellini (1996a,b); Stockman (2001); Besley and Coate (2003); Besley and Smart (2007); Caballero and Yared (2010); Yared (2010); Halac and Yared (2014); Azzimonti, Battaglini and Coate (2015).
} 


\section{Model}

Consider a stylized economy and political system with two parties labeled $A$ and $B$. There are two time periods indexed by $t \in\{1,2\} .{ }^{10}$ In each period $t$, the two parties decide an allocation to a public good $x_{t} \in \mathbb{R}_{+}$. The stage utility for party $i \in\{A, B\}$ in period $t$ is $u_{i t}\left(x_{t}\right)$. Party $i$ seeks to maximize its dynamic payoff from the sequence of public good allocations $u_{i 1}\left(x_{1}\right)+\delta u_{i 2}\left(x_{2}\right)$, where $\delta \in(0,1]$ is the parties' common discount factor.

We assume $u_{i t}$ is twice continuously differentiable, strictly concave, and attains a maximum at $\theta_{i t}$ for all $i \in\{A, B\}$ and $t \in\{1,2\}$. This implies $u_{i t}$ is single-peaked with $\theta_{i t}$ denoting party $i$ 's ideal level of the public good in period $t .{ }^{11}$ We assume parties' ideal levels of the public good are positive and party $A$ 's ideal is lower than party $B$ 's. That is, $0<\theta_{A t} \leq \theta_{B t}$ for all $t$. Parties' ideal levels of the public good may vary across periods. In particular, they may be increasing with $\theta_{i 1}<\theta_{i 2}$ for all $i \in\{A, B\}$, decreasing with $\theta_{i 1}>\theta_{i 2}$ for all $i \in\{A, B\}$, divergent with $\theta_{A 2}<\theta_{A 1}<\theta_{B 1}<\theta_{B 2}$, or convergent with $\theta_{A 1}<\theta_{A 2}<\theta_{B 2}<\theta_{B 1}$.

We consider a political system with unanimity rule. ${ }^{12}$ At the beginning of each period, a party is randomly selected to make a proposal for the allocation to the public good. The probability that party $i$ proposes in a period is $p_{i} \in(0,1) .{ }^{13}$ Spending on the public good may be allocated by way of different programs - a discretionary program, which expires after one period, or a mandatory program, for which spending will continue in the next period unless the parties agree to change it. Denote the proposed amount allocated to a discretionary program in period $t$ as $k_{t}$, and to a mandatory program as $g_{t}$. If the responding party agrees to the proposal, the implemented allocation to the public good for the period is the sum of the discretionary and mandatory allocations proposed, so $x_{t}=k_{t}+g_{t}$; otherwise, $x_{t}=g_{t-1}$.

\footnotetext{
${ }^{10}$ In Section 7 we consider a more general model with any number of periods and random preferences.

${ }^{11}$ Because of the opportunity cost of providing public goods, it is reasonable to model parties' utility functions as single-peaked as in, for example, Baron (1996).

${ }^{12}$ Most political systems are not formally characterized by unanimity rule, however, many have institutions that limit a single party's power, for example, the "checks and balances" included in the U.S. Constitution. Under these institutions, if the majority party's power is not sufficiently high, then it needs approval of the other party to set new policies.

${ }^{13}$ More generally, the probability that party $i$ proposes in period $t$ is $p_{i t}$. In the two-period model, $p_{i 1}$ does not play a role, and for notational simplicity we write $p_{i}$ as the probability that party $i$ proposes in period 2. In Section 7 we extend our model to an arbitrary time horizon and we use the general notation.
} 
Denote a proposal by $z_{t}=\left(k_{t}, g_{t}\right)$. We require $g_{t} \geq 0$ to ensure a positive status quo each period. Let $Z \subseteq \mathbb{R} \times \mathbb{R}_{+}$be the set of feasible proposals. The set $Z$ is determined by the rules governing mandatory and discretionary programs, and hence we call $Z$ the budgetary institution. We consider the following institutions: only discretionary programs, in which case $Z=\mathbb{R}_{+} \times\{0\}$ and $g_{t-1}=0$ for all $t$; only mandatory programs, in which case $Z=\{0\} \times \mathbb{R}_{+} ;$ both mandatory and positive discretionary, in which case $Z=\mathbb{R}_{+} \times \mathbb{R}_{+}$; and both mandatory and discretionary where discretionary spending may be positive or negative, in which case $Z=\left\{\left(k_{t}, g_{t}\right) \in \mathbb{R} \times \mathbb{R}_{+} \mid k_{t}+g_{t} \geq 0\right\}$. It is natural to think of spending as positive, but it is also possible to have temporary cuts to spending on mandatory programs, for example government furloughs that temporarily reduce salaries of public employees. This temporary reduction in mandatory spending can be thought of as negative discretionary spending as it reduces total spending in the current period, but does not affect the status quo for the next period.

A pure strategy for party $i$ in period $t$ is a pair of functions $\sigma_{i t}=\left(\pi_{i t}, \alpha_{i t}\right)$, where $\pi_{i t}: \mathbb{R}_{+} \rightarrow Z$ is a proposal strategy for party $i$ in period $t$ and $\alpha_{i t}: \mathbb{R}_{+} \times Z \rightarrow\{0,1\}$ is an acceptance strategy for party $i$ in period $t .{ }^{14}$ Party $i$ 's proposal strategy $\pi_{i t}=\left(\kappa_{i t}, \gamma_{i t}\right)$ associates with each status quo $g_{t-1}$ an amount of public good spending in discretionary programs, denoted by $\kappa_{i t}\left(g_{t-1}\right)$, and an amount in mandatory programs, denoted by $\gamma_{i t}\left(g_{t-1}\right)$. Party $i$ 's acceptance strategy $\alpha_{i t}\left(g_{t-1}, z_{t}\right)$ takes the value 1 if party $i$ accepts the proposal $z_{t}$ offered by the other party when the status quo is $g_{t-1}$, and 0 otherwise. ${ }^{15}$

We consider subgame perfect equilibria and restrict attention to equilibria in which (i) $\alpha_{i t}\left(g_{t-1}, z_{t}\right)=1$ when party $i$ is indifferent between $g_{t-1}$ and $z_{t}$; and (ii) $\alpha_{i t}\left(g_{t-1}, \pi_{j t}\left(g_{t-1}\right)\right)=1$ for all $t, g_{t-1} \in \mathbb{R}_{+}, i, j \in\{A, B\}$ with $j \neq i$. That is, the responder accepts any proposal that it is indifferent between accepting and rejecting, and the equilibrium proposals are always

\footnotetext{
${ }^{14}$ In this two-period model, we show that equilibrium strategies in the second period are unique. Thus, in equilibrium, the second-period strategy does not depend on the history except through the status quo, so writing strategies as depending on history only through the status quo is without loss of generality. This result extends to the finite-horizon case of state-contingent mandatory spending considered in Section 7. For the infinite-horizon case, the restriction on strategies implies a Markov restriction on the equilibrium.

${ }^{15} \mathrm{We}$ are interested in efficiency properties of budgetary institutions. Because the utility functions are strictly concave, Pareto efficient allocations do not involve randomization. Hence, if any pure strategy equilibrium is inefficient, allowing mixed strategies does not improve efficiency.
} 
accepted. ${ }^{16}$ We henceforth refer to a subgame perfect equilibrium that satisfies (i) and (ii) simply as an equilibrium.

Denote an equilibrium by $\sigma^{*}$. Let party $i \in\{A, B\}$ be the proposer and party $j \in\{A, B\}$ be the responder in period 2. (When we use $i$ to denote the proposer and $j$ to denote the responder without any qualifier, it is understood that $i \neq j$.) Given conditions (i) and (ii), for any $g_{1}$ admissible under $Z$, the equilibrium proposal strategy $\left(\kappa_{i 2}^{*}\left(g_{1}\right), \gamma_{i 2}^{*}\left(g_{1}\right)\right)$ of party $i$ in period 2 solves

$$
\begin{aligned}
\max _{\left(k_{2}, g_{2}\right) \in Z} & u_{i 2}\left(k_{2}+g_{2}\right) \\
\text { s.t. } & u_{j 2}\left(k_{2}+g_{2}\right) \geq u_{j 2}\left(g_{1}\right) .
\end{aligned}
$$

Let $V_{i}\left(g ; \sigma_{2}\right)$ be the expected second-period payoff for party $i$ given first-period mandatory spending $g$ and second-period strategies $\sigma_{2}=\left(\sigma_{A 2}, \sigma_{B 2}\right)$. That is

$$
V_{i}\left(g ; \sigma_{2}\right)=p_{A} u_{i 2}\left(\kappa_{A 2}(g)+\gamma_{A 2}(g)\right)+p_{B} u_{i 2}\left(\kappa_{B 2}(g)+\gamma_{B 2}(g)\right) .
$$

If party $i$ is the proposer and party $j$ is the responder in period 1 , then for any $g_{0}$ admissible under $Z$ the equilibrium proposal strategy $\left(\kappa_{i 1}^{*}\left(g_{0}\right), \gamma_{i 1}^{*}\left(g_{0}\right)\right)$ of party $i$ in period 1 solves

$$
\begin{aligned}
\max _{\left(k_{1}, g_{1}\right) \in Z} & u_{i 1}\left(k_{1}+g_{1}\right)+\delta V_{i}\left(g_{1} ; \sigma_{2}^{*}\right) \\
\text { s.t. } & u_{j 1}\left(k_{1}+g_{1}\right)+\delta V_{j}\left(g_{1} ; \sigma_{2}^{*}\right) \geq u_{j 1}\left(g_{0}\right)+\delta V_{j}\left(g_{0} ; \sigma_{2}^{*}\right) .
\end{aligned}
$$

\section{$3 \quad$ Pareto efficiency}

In this section we characterize Pareto efficient allocations and define Pareto efficient equilibria, both in the static and the dynamic sense.

\subsection{Pareto efficient allocations}

We distinguish between the social planner's static problem (SSP), which determines static Pareto efficient allocations, and the social planner's dynamic problem (DSP), which determines dynamic Pareto efficient allocations.

We define a statically Pareto efficient allocation in period $t$ as the solution to the following

\footnotetext{
${ }^{16}$ Any equilibrium is payoff equivalent to some equilibrium (possibly itself) that satisfies (i) and (ii). Similar restrictions are made in Bowen, Chen and Eraslan (2014) and the proof follows the same arguments as in that paper. We omit the arguments here for space considerations.
} 
maximization problem

$$
\begin{aligned}
\max _{x_{t} \in \mathbb{R}_{+}} & u_{i t}\left(x_{t}\right) \\
\text { s.t. } & u_{j t}\left(x_{t}\right) \geq \bar{u}
\end{aligned}
$$

for some $\bar{u} \in \mathbb{R}, i, j \in\{A, B\}$ and $i \neq j .{ }^{17}$ By Proposition 1, statically Pareto efficient allocations are all those between the ideal points of the parties.

Proposition 1. An allocation $x_{t}$ is statically Pareto efficient in period $t$ if and only if $x_{t} \in$ $\left[\theta_{A t}, \theta_{B t}\right]$.

Denote a sequence of allocations by $\mathbf{x}=\left(x_{1}, x_{2}\right)$ and party $i$ 's discounted dynamic payoff from $\mathbf{x}$ by $U_{i}(\mathbf{x})=\sum_{t=1}^{2} \delta^{t-1} u_{i t}\left(x_{t}\right)$. We define a dynamically Pareto efficient allocation as the solution to the following maximization problem

$$
\begin{aligned}
\max _{\mathbf{x} \in \mathbb{R}_{+}^{2}} & U_{i}(\mathbf{x}) \\
\text { s.t. } & U_{j}(\mathbf{x}) \geq \bar{U}
\end{aligned}
$$

for some $\bar{U} \in \mathbb{R}, i, j \in\{A, B\}$ and $i \neq j$. Denote the sequence of party $i$ 's static ideals by $\boldsymbol{\theta}_{i}=\left(\theta_{i 1}, \theta_{i 2}\right)$ for all $i \in\{A, B\}$, and denote the solution to (DSP) as $\mathbf{x}^{*}=\left(x_{1}^{*}, x_{2}^{*}\right){ }^{18}$ Proposition 2 characterizes the dynamically Pareto efficient allocations. ${ }^{19}$

Proposition 2. A dynamically Pareto efficient allocation $\mathbf{x}^{*}$ satisfies the following properties:

1. For all $t, x_{t}^{*}$ is statically Pareto efficient. That is, $x_{t}^{*} \in\left[\theta_{A t}, \theta_{B t}\right]$ for all $t$.

2. Either $\mathbf{x}^{*}=\boldsymbol{\theta}_{A}$, or $\mathbf{x}^{*}=\boldsymbol{\theta}_{B}$, or $u_{A t}^{\prime}\left(x_{t}^{*}\right)+\lambda^{*} u_{B t}^{\prime}\left(x_{t}^{*}\right)=0$ for some $\lambda^{*}>0$, for all $t$.

Proposition 2 part 2 implies that if $\mathbf{x}^{*} \neq \boldsymbol{\theta}_{i}$ for all $i \in\{A, B\}$, and $\theta_{A t} \neq \theta_{B t}$ in period $t$ then we must have

$$
-\frac{u_{A t}^{\prime}\left(x_{t}^{*}\right)}{u_{B t}^{\prime}\left(x_{t}^{*}\right)}=\lambda^{*}
$$

\footnotetext{
${ }^{17}$ The social planner's static problem (SSP) is a standard concave programming problem so the solution is unique for a given $\bar{u}$ if it exists.

${ }^{18}$ Note the solution to (DSP) depends on $\bar{U}$, but for notational simplicity we suppress this dependency and denote the solution to (DSP) as $\mathbf{x}^{*}$. The solution to (DSP) is unique for a given $\bar{U}$ if it exists.

${ }^{19}$ In the proof of Proposition 2 in the Appendix, we generalize (DSP) to any number of periods and prove Proposition 2 for this more general problem.
} 
for some $\lambda^{*}>0 .{ }^{20} \mathrm{By}(1)$ if parties $A$ and $B$ do not have the same ideal level of the public good in periods 1 and 2, then in a dynamically Pareto efficient allocation, either the allocation is equal to party $A$ 's or party $B$ 's ideal in both periods, or the ratio of their marginal utilities is equal across these two periods, i.e., $\frac{u_{A 1}^{\prime}\left(x_{1}^{*}\right)}{u_{B 1}^{\prime}\left(x_{1}^{*}\right)}=\frac{u_{A 2}^{\prime}\left(x_{2}^{*}\right)}{u_{B 2}^{\prime}\left(x_{2}^{*}\right)}$. The intuition for the latter is that if the ratio of marginal utilities is not constant across periods, then there is an intertemporal reallocation such that at least one party is strictly better off and the other party is no worse off. In both cases there is a dynamic link across periods.

\subsection{Pareto efficient equilibrium}

We define a dynamically Pareto efficient equilibrium given an initial status quo $g_{0}$ as an equilibrium that results in a dynamically Pareto efficient allocation for any realization of the sequence of proposers. More precisely, denote an equilibrium strategy profile as $\sigma^{*}=\left(\left(\sigma_{A 1}^{*}, \sigma_{A 2}^{*}\right),\left(\sigma_{B 1}^{*}, \sigma_{B 2}^{*}\right)\right)$ with $\sigma_{i t}^{*}=\left(\left(\kappa_{i t}^{*}, \gamma_{i t}^{*}\right), \alpha_{i t}^{*}\right)$. An equilibrium allocation for $\sigma^{*}$ given initial status quo $g_{0}$ is a possible realization of total public good spending for each period $\mathbf{x}^{\sigma^{*}}\left(g_{0}\right)=\left(x_{1}^{\sigma^{*}}\left(g_{0}\right), x_{2}^{\sigma^{*}}\left(g_{0}\right)\right)$, where $x_{1}^{\sigma^{*}}\left(g_{0}\right)=\kappa_{i 1}^{*}\left(g_{0}\right)+\gamma_{i 1}^{*}\left(g_{0}\right)$, and $x_{2}^{\sigma^{*}}\left(g_{0}\right)=$ $\kappa_{j 2}^{*}\left(\gamma_{i 1}^{*}\left(g_{0}\right)\right)+\gamma_{j 2}^{*}\left(\gamma_{i 1}^{*}\left(g_{0}\right)\right)$ for some $i, j \in\{A, B\}$. The random determination of proposers induces a probability distribution over allocations given an equilibrium $\sigma^{*}$. Thus any element in the support of this distribution is an equilibrium allocation for $\sigma^{*}{ }^{21}$ We require every allocation in the support of this distribution to be dynamically Pareto efficient for the equilibrium to be dynamically Pareto efficient.

Definition 1. An equilibrium $\sigma^{*}$ is a dynamically Pareto efficient equilibrium given initial status quo $g_{0}$ if and only if every equilibrium allocation $\mathbf{x}^{\sigma^{*}}\left(g_{0}\right)$ for $\sigma^{*}$ given initial status quo $g_{0}$ is dynamically Pareto efficient.

A statically Pareto efficient equilibrium given initial status quo $g_{0}$ is analogously defined as an equilibrium in which the realized allocation to the public good is statically Pareto efficient in all periods $t$ given initial status quo $g_{0}$. Thus a necessary condition for $\sigma^{*}$ to be a

\footnotetext{
${ }^{20}$ This is because if $u_{B t}^{\prime}\left(x_{t}^{*}\right)=0$, then part 2 of Proposition 2 implies that we must also have $u_{A t}^{\prime}\left(x_{t}^{*}\right)=0$ which is not possible when $\theta_{A t} \neq \theta_{B t}$.

${ }^{21}$ For example, if $A$ is the proposer in period 1 and $B$ is the proposer in period 2, then the equilibrium allocation is $x_{1}^{\sigma^{*}}\left(g_{0}\right)=\kappa_{A 1}^{*}\left(g_{0}\right)+\gamma_{A 1}^{*}\left(g_{0}\right)$, and $x_{2}^{\sigma^{*}}\left(g_{0}\right)=\kappa_{B 2}^{*}\left(\gamma_{A 1}^{*}\left(g_{0}\right)\right)+\gamma_{B 2}^{*}\left(\gamma_{A 1}^{*}\left(g_{0}\right)\right)$.
} 
dynamically Pareto efficient equilibrium is that $\sigma^{*}$ is a statically Pareto efficient equilibrium.

The analysis of efficiency properties of equilibria under different budgetary institutions is aided by the following results. We first show that if parties' ideal levels of the public good are different in both periods, then given a spending level in the first period, a unique spending level in the second period is required for the allocation to be dynamically Pareto efficient. Given strict concavity of the utility functions, the solution to equation (1) for a fixed $\lambda^{*}$ is unique, and thus given some allocation in period $t^{\prime}$, the allocations in all other periods are uniquely pinned down. This means that if the equilibrium level of spending in period 2 varies with the identity of the period-2 proposer, then the equilibrium cannot be dynamically Pareto efficient. Thus dynamically Pareto efficient equilibria avoid political risk. We formalize this in Lemma $1 .^{22}$

Lemma 1. Suppose $\theta_{A t} \neq \theta_{B t}$ for all $t$. If allocations $\mathbf{x}$ and $\tilde{\mathbf{x}}$ are both dynamically Pareto efficient and $x_{t^{\prime}}=\tilde{x}_{t^{\prime}}$ for some $t^{\prime}$, then $\mathbf{x}=\tilde{\mathbf{x}}$.

We next show that dynamic Pareto efficiency typically requires variation across periods when parties' ideals are evolving. We say that the parties are in gridlock if the spending in period 2 does not change when preferences are different from period 1. Thus dynamically Pareto efficient allocations typically avoid gridlock. We formalize this result in Lemma 2. If $\theta_{i 1} \neq \theta_{i 2}$, we can write $u_{i t}\left(x_{t}\right)=u_{i}\left(x_{t}, \theta_{i t}\right)$, and we say that utilities have increasing marginal returns if $\frac{\partial u_{i}}{\partial x_{t}}$ is strictly increasing in $\theta_{i t}$ for all $t, x_{t}$ and $i \in\{A, B\} .{ }^{23}$ The increasing marginal returns property ensures that if both parties' ideals increase (or decrease) over time, then the spending level in any dynamically Pareto efficient allocation must also increase (or decrease) over time. ${ }^{24}$ This property is satisfied by commonly used utility functions such as

\footnotetext{
${ }^{22}$ Note that our definition of a dynamically Pareto efficient equilibrium requires ex-post dynamic Pareto efficiency, that is, allocations must be dynamically Pareto efficient for each realized path of proposers. Two other notions of a dynamically Pareto efficient equilibrium might be considered: Ex-ante dynamic Pareto efficiency, before the realization of the first-period proposer, and interim dynamic Pareto efficiency, after the realization of the first-period proposer but before the realization of the second-period proposer. By Lemma 1 , if $\theta_{A t} \neq \theta_{B t}$ for any $t$, then ex-post efficiency implies interim efficiency. We can interpret our results as showing which budgetary institutions result in dynamically Pareto efficient allocations conditional on the initial party in power. Ex-ante Pareto efficiency would require the first-period allocation to be invariant to the party in power, a stronger requirement than ex-post or interim Pareto efficiency.

${ }^{23}$ Increasing marginal returns is stronger than strict supermodularity (e.g., Edlin and Shannon, 1998).

${ }^{24}$ This is stated formally in Lemma A1 in the Appendix.
} 
$u_{i t}\left(x_{t}\right)=-\left(x_{t}-\theta_{i t}\right)^{2}$.

Lemma 2. Under any of the following conditions, we have $x_{1}^{*} \neq x_{2}^{*}$ :

1. Parties' ideals are increasing or decreasing and not overlapping, that is, $\theta_{A 1}<\theta_{B 1}<$ $\theta_{A 2}<\theta_{B 2}$ or $\theta_{A 2}<\theta_{B 2}<\theta_{A 1}<\theta_{B 1}$.

2. Parties' ideals are increasing or decreasing, and utilities have increasing marginal returns.

3. Parties' ideals are divergent or convergent, $u_{i t}\left(x_{t}\right)=-\left(\left|x_{t}-\theta_{i t}\right|\right)^{r}$ with $r>1$ for all $t$ and $i \in\{A, B\}$ and $x_{1}^{*} \neq \frac{\theta_{A 1} \theta_{B 2}-\theta_{B 1} \theta_{A 2}}{\theta_{B 2}-\theta_{B 1}+\theta_{A 1}-\theta_{A 2}}$.

\section{Discretionary spending}

Suppose spending is allocated through discretionary programs only, implying that the status quo in each period is exogenous and equal to zero. In this case there is no dynamic link between the previous period's policy and the current period's status quo, and $Z=\mathbb{R}_{+} \times\{0\}$. Without the dynamic link between periods, the bargaining between the two parties is a static problem, similar to the monopoly agenda-setting model in Romer and Rosenthal (1978, 1979). ${ }^{25}$ For this section we denote a proposal in period $t$ by $k_{t}$ since $g_{t}$ is zero. Consider any period $t$. Since $u_{B t}$ is single-peaked at $\theta_{B t}$ and $0<\theta_{A t} \leq \theta_{B t}$, we have $u_{B t}(0)<u_{B t}\left(\theta_{A t}\right)$. Hence, if party $A$ is the proposer in period $t$, it proposes its ideal policy $k_{t}=\theta_{A t}$, which is accepted by party $B$. If party $B$ is the proposer in period $t$, however, whether it can implement its ideal policy depends on the locations of the parties' ideal points relative to the status quo. Specifically, let $\phi_{A t}^{o}$ be the highest policy that makes party $A$ as well off as the status quo in period $t$. That is,

$$
\phi_{A t}^{o}=\max \left\{x \in \mathbb{R}_{+} \mid u_{A t}(x) \geq u_{A t}(0)\right\} .
$$

Note that $\phi_{A t}^{o}>\theta_{A t}$. We next characterize the equilibrium and its efficiency properties.

${ }^{25}$ Note that when only discretionary spending is allowed, $g_{0}=g_{1}=0$, and hence the proposer's first-period problem $\left(P_{1}\right)$ becomes a static problem identical to $\left(P_{2}\right)$. 
Proposition 3. Under a budgetary institution that allows only discretionary spending programs, given the initial status quo of zero:

1. The equilibrium is statically Pareto efficient.

2. The equilibrium is dynamically Pareto inefficient if and only if $\theta_{A t} \neq \theta_{B t}$ for all $t$.

Specifically, the equilibrium level of spending in period $t$ is $x_{t}=\theta_{A t}$ if party $A$ is the proposer and is $x_{t}=\min \left\{\theta_{B t}, \phi_{A t}^{o}\right\} \in\left[\theta_{A t}, \theta_{B t}\right]$ if party $B$ is the proposer.

The efficiency properties can be seen from the equilibrium strategies. Static efficiency obtains since the equilibrium spending is in the interval $\left[\theta_{A t}, \theta_{B t}\right]$ for all $t$. Dynamic inefficiency arises if $\theta_{A t} \neq \theta_{B t}$ for all $t$ because the equilibrium spending level depends on the identity of the proposer, hence there is dynamic inefficiency due to political risk by Lemma $1 .{ }^{26}$ When $\theta_{A t} \neq \theta_{B t}$ for at most one period, dynamic efficiency is equivalent to static efficiency. This is because the restriction in (1) does not apply when $\theta_{A t}=\theta_{B t}$, hence it does not place a dynamic restriction on spending levels. ${ }^{27}$

\section{$5 \quad$ Mandatory spending}

When mandatory programs are allowed, the proposing party in the first period takes into account the effect of the amount allocated to the mandatory program on the secondperiod spending because it becomes the status quo in the second period. This creates a dynamic link between periods. We show that this dynamic game admits an equilibrium and give properties of the equilibrium proposal strategies in period $2 .{ }^{28}$ The result applies to any budgetary institution that allows mandatory spending programs, in combination with discretionary spending or in isolation. We formalize this result in the next subsection.

\footnotetext{
${ }^{26}$ Political risk is the only source of inefficiency in part because the status quo spending in the discretionary only institution is exogenously zero, and hence always lower than both parties' ideal points. If the exogenous status quo is between the ideal points in both periods, then the source of dynamic inefficiency is gridlock because in equilibrium the spending is stuck at the status quo. We find it natural that in our model of public spending the exogenous status quo is fixed at zero.

${ }^{27}$ The result is stated for the two-period model, but a straightforward generalization of the argument shows that in a model with an arbitrary number of periods, Proposition 3 holds if the condition in part 2 is replaced by $\theta_{A t} \neq \theta_{B t}$ for at least two periods.

${ }^{28}$ Equilibrium existence is not immediate because lower hemicontinuity of the second-period acceptance correspondence requires a non-trivial proof. The proof is given in the Appendix.
} 


\subsection{Preliminaries}

Define the functions $\phi_{A t}$ and $\phi_{B t}$ which are analogous to $\phi_{A t}^{o}$. The value $\phi_{A t}\left(g_{t-1}\right)$ is the highest acceptable spending level to party $A$ and $\phi_{B t}\left(g_{t-1}\right)$ is the lowest acceptable spending level to party $B$ when the status quo is $g_{t-1}$. That is,

$$
\begin{aligned}
& \phi_{A t}\left(g_{t-1}\right)=\max \left\{x \in \mathbb{R}_{+} \mid u_{A t}(x) \geq u_{A t}\left(g_{t-1}\right)\right\}, \\
& \phi_{B t}\left(g_{t-1}\right)=\min \left\{x \in \mathbb{R}_{+} \mid u_{B t}(x) \geq u_{B t}\left(g_{t-1}\right)\right\} .
\end{aligned}
$$

These are illustrated below in Figure 1. If $g_{t-1}<\theta_{A t}$, then $\phi_{A t}\left(g_{t-1}\right)>g_{t-1}$ and if $g_{t-1} \geq$ $\theta_{A t}$, then $\phi_{A t}\left(g_{t-1}\right)=g_{t-1}$. If $g_{t-1} \leq \theta_{B t}$, then $\phi_{B t}\left(g_{t-1}\right)=g_{t-1}$ and if $g_{t-1}>\theta_{B t}$, then $\phi_{B t}\left(g_{t-1}\right)<g_{t-1}$.

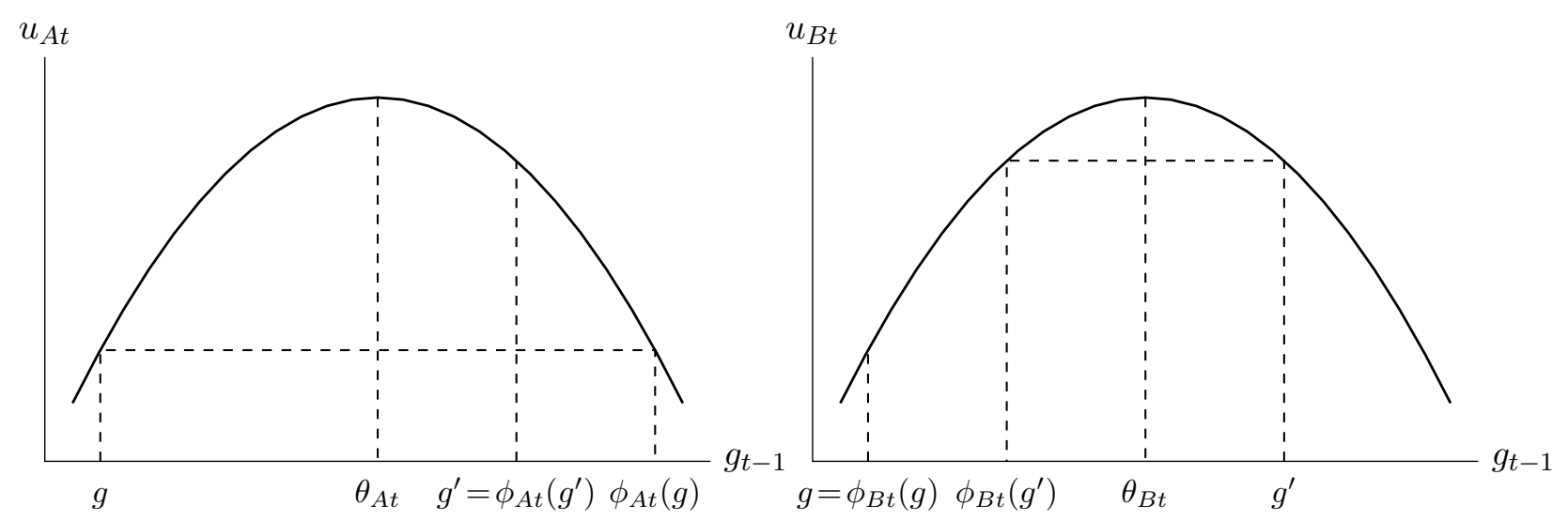

Figure 1: $\phi_{A t}$ and $\phi_{B t}$

Proposition 4. Under any budgetary institution that allows mandatory spending programs, an equilibrium exists. For any $g_{1} \in \mathbb{R}_{+}$, the equilibrium spending in period 2 is unique and satisfies

Furthermore:

$$
\begin{aligned}
& \kappa_{A 2}^{*}\left(g_{1}\right)+\gamma_{A 2}^{*}\left(g_{1}\right)=\max \left\{\theta_{A 2}, \phi_{B 2}\left(g_{1}\right)\right\}, \\
& \kappa_{B 2}^{*}\left(g_{1}\right)+\gamma_{B 2}^{*}\left(g_{1}\right)=\min \left\{\theta_{B 2}, \phi_{A 2}\left(g_{1}\right)\right\} .
\end{aligned}
$$

1. $\kappa_{i 2}^{*}\left(g_{1}\right)+\gamma_{i 2}^{*}\left(g_{1}\right) \in\left[\theta_{A 2}, \theta_{B 2}\right]$ for all $i \in\{A, B\}$ and all $g_{1} \in \mathbb{R}_{+}$. 
2. If $\theta_{A 2} \neq \theta_{B 2}$, then

$$
\begin{array}{ll}
\kappa_{A 2}^{*}\left(g_{1}\right)+\gamma_{A 2}^{*}\left(g_{1}\right)=\kappa_{B 2}^{*}\left(g_{1}\right)+\gamma_{B 2}^{*}\left(g_{1}\right)=g_{1} & \text { if } g_{1} \in\left[\theta_{A 2}, \theta_{B 2}\right], \\
\kappa_{A 2}^{*}\left(g_{1}\right)+\gamma_{A 2}^{*}\left(g_{1}\right)<\kappa_{B 2}^{*}\left(g_{1}\right)+\gamma_{B 2}^{*}\left(g_{1}\right) & \text { if } g_{1} \notin\left[\theta_{A 2}, \theta_{B 2}\right] .
\end{array}
$$

Proposition 4 gives the equilibrium level of total spending in the second period, which is unique for any status quo. Part 1 implies that the equilibrium level of spending in period 2 is statically Pareto efficient. Part 2 gives properties of the equilibrium spending if the parties' ideals are different. If the status quo is statically Pareto efficient, then it is maintained. If the status quo is not statically Pareto efficient, then the equilibrium proposal is different from the status quo and depends on the identity of the proposer - specifically, it is lower when $A$ is the proposer than when $B$ is the proposer.

Figure 2 is an example of equilibrium spending in period 2 for quadratic loss utility. While the exact form depends on the specific utility function, any second-period strategy has similar properties. Consider party $A$ as the proposer in period 2. If $g_{1}<\theta_{A 2}$, then party $A$ proposes its ideal policy $x_{2}=\theta_{A 2}$, which is accepted since $u_{B 2}\left(g_{1}\right)<u_{B 2}\left(\theta_{A 2}\right)$. If $g_{1} \in\left[\theta_{A 2}, \theta_{B 2}\right]$, then party $A$ proposes $x_{2}=g_{1}$ since any $x_{2}<g_{1}$ would be rejected by party $B$ and party $A$ prefers $g_{1}$ to any $x_{2}>g_{1}$. If $g_{1}>\theta_{B 2}$, then party $B$ accepts all proposals in the interval $\left[\phi_{B 2}\left(g_{1}\right), g_{1}\right]$ since these are closer to $\theta_{B 2}$ than $g_{1}$ is. Since $\theta_{A 2}<\theta_{B 2}<g_{1}$, either $\theta_{A 2} \in\left[\phi_{B 2}\left(g_{1}\right), g_{1}\right]$ or $\theta_{A 2}<\phi_{B 2}\left(g_{1}\right)$. If $\theta_{A 2} \in\left[\phi_{B 2}\left(g_{1}\right), g_{1}\right]$, then party $A$ proposes $x_{2}=\theta_{A 2}$. If $\theta_{A 2}<\phi_{B 2}\left(g_{1}\right)$, then party $A$ proposes the policy closest to $\theta_{A 2}$ that is acceptable to $B$, which is $\phi_{B 2}\left(g_{1}\right)$. For quadratic loss utility function, if $g_{1} \geq \theta_{B 2}$ and $\phi_{B 2}\left(g_{1}\right) \geq \theta_{A 2}$, we have $\phi_{B 2}\left(g_{1}\right)=2 \theta_{B 2}-g_{1}$, which is linear and decreasing in $g_{1}$. For general strictly concave $u_{B 2}$, if $g_{1} \geq \theta_{B 2}$ and $\phi_{B 2}\left(g_{1}\right) \geq \theta_{A 2}$, then $\phi_{B 2}\left(g_{1}\right)$ is decreasing in $g_{1}$, but may not be linear.

Figure 2 indicates the potential sources of dynamic inefficiency with mandatory programs. If the status quo is in $\left[\theta_{A 2}, \theta_{B 2}\right]$, then the period- 2 spending is equal to the status quo, so there is potential for gridlock. If the status quo is outside $\left[\theta_{A 2}, \theta_{B 2}\right]$, then the period-2 spending depends on the identity of the proposer and political risk is a source of dynamic inefficiency. We show in the next section that with only mandatory spending programs at least one of these sources of dynamic inefficiency arises, except in special cases. 


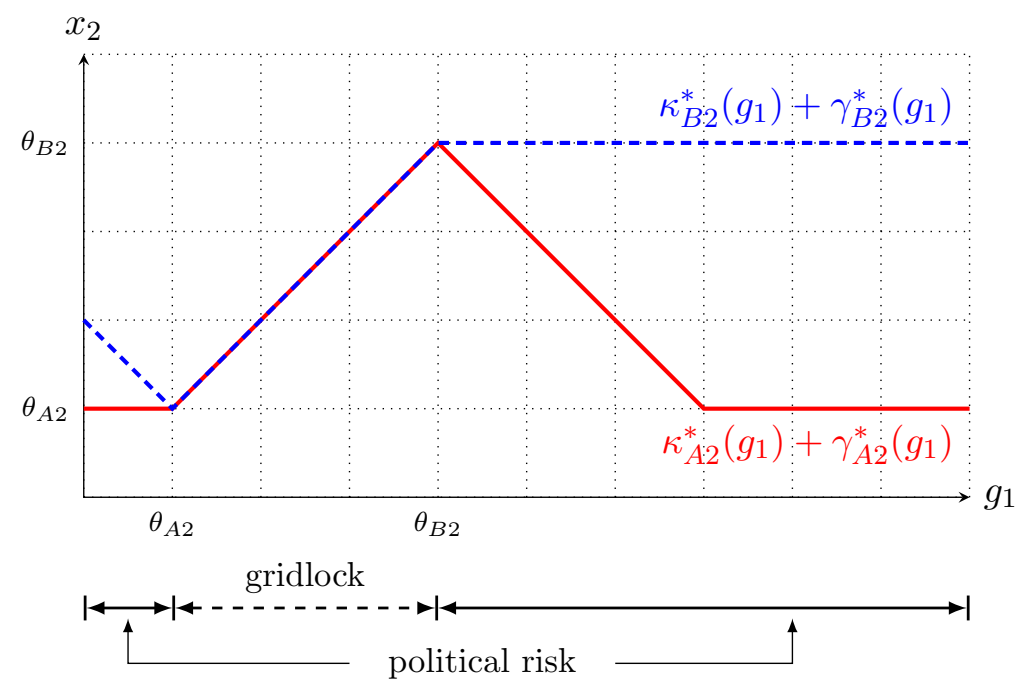

Figure 2: Period-2 equilibrium strategies with mandatory spending for $u_{i t}\left(x_{t}\right)=-\left(x_{t}-\theta_{i t}\right)^{2}$

\section{$5.2 \quad$ Inefficiency with mandatory spending only}

Suppose now that spending is allocated through mandatory programs only, that is, $Z=$ $\{0\} \times \mathbb{R}_{+}$. Since $k_{t}$ is zero for any $t$, the equilibrium discretionary proposal $\kappa_{i t}^{*}\left(g_{t-1}\right)$ is zero for all $i \in\{A, B\}$, all $t$ and all $g_{t-1} \in \mathbb{R}_{+}$. For the rest of the section we thus denote a proposal in period $t$ by $g_{t}$.

We begin by noting that equilibrium allocations can be dynamically Pareto efficient in the absence of a conflict in period 2 or in the absence of variation in ideal levels of public good spending, but these are special cases. ${ }^{29}$ We show that equilibrium allocations are in general dynamically Pareto inefficient and can even be statically Pareto inefficient. Others have shown inefficiency with an endogenous status quo and evolving preferences in settings different from ours. ${ }^{30}$ By demonstrating inefficiency with mandatory spending only in our setting, we highlight its sources: political risk and gridlock. This helps to understand how appropriate flexibility in mandatory spending avoids inefficiency, which we show in the next section. Proposition 5 gives conditions under which equilibria are dynamically Pareto inefficient with mandatory spending only.

\footnotetext{
${ }^{29}$ We show this in Section A3.4 in the Appendix.

${ }^{30}$ For example, Riboni and Ruge-Murcia (2008) show dynamic inefficiency in the context of central bank decision-making, and Zapal (2011) and Dziuda and Loeper (2015) show static inefficiency in other settings.
} 
Proposition 5. Under a budgetary institution that allows only mandatory spending programs, any equilibrium $\sigma^{*}$ is dynamically Pareto inefficient for any initial status quo $g_{0} \in \mathbb{R}_{+}$, if either of the following conditions holds:

1. Parties' ideals are increasing or decreasing and not overlapping, that is $\theta_{A 1}<\theta_{B 1}<$ $\theta_{A 2}<\theta_{B 2}$ or $\theta_{A 2}<\theta_{B 2}<\theta_{A 1}<\theta_{B 1}$.

2. Parties' ideals are increasing or decreasing, $\theta_{A t} \neq \theta_{B t}$ for all $t$ and utilities have increasing marginal returns.

Furthermore, if parties' ideals are divergent or convergent and $u_{i t}\left(x_{t}\right)=-\left(\left|x_{t}-\theta_{i t}\right|\right)^{r}$ with $r>1$, then for any equilibrium $\sigma^{*}$, there exists a unique $g_{0}$ such that $\sigma^{*}$ is dynamically Pareto efficient if the initial status quo is $g_{0}$.

Proposition 5 parts 1 and 2 give conditions under which equilibria are dynamically Pareto inefficient for all initial status quos, when parties' ideals are increasing or decreasing. The final part states that when preferences are divergent or convergent, for a certain class of utility functions, dynamic efficiency is obtained only for a unique initial status quo. ${ }^{31}$

To gain some intuition for Proposition 5, note that because of the second-period conflict between the two parties, either the level of public good spending in period 2 varies with the identity of the proposing party, which results in political risk, or neither party changes the status quo, which results in gridlock. ${ }^{32}$

The next result shows that equilibrium allocations under mandatory spending programs can violate not only dynamic, but also static Pareto efficiency.

\footnotetext{
${ }^{31}$ Dynamic inefficiency also obtains in a finite-horizon model with more than two periods under the conditions in Proposition 5, when the fluctuations in preferences apply to the last two periods.

${ }^{32}$ The only source of dynamic inefficiency with discretionary only institutions is political risk while either political risk or gridlock is a potential source of dynamic inefficiency with mandatory only institutions. With political turnover but stable preferences, gridlock is not a source of inefficiency. In this environment political risk does not arise with mandatory only institutions as shown in Proposition A1, and hence these achieve dynamic Pareto efficiency. In the same environment, discretionary only institutions do not eliminate political risk and are inefficient by Proposition 3. Conversely, in an environment without political turnover but evolving preferences, discretionary only institutions can be dynamically Pareto efficient, whereas mandatory only institutions are generically inefficient.
} 
Proposition 6. Suppose $u_{i t}\left(x_{t}\right)=-\left(x_{t}-\theta_{i t}\right)^{2}$ for all $i \in\{A, B\}$ and all $t$. Under a budgetary institution that allows only mandatory spending programs, if either $\theta_{A 2}<\theta_{A 1}<\theta_{B 2}$ or $\theta_{A 2}<\theta_{B 1}<\theta_{B 2}$, then there exists a nonempty open interval $\mathcal{I}$ such that any equilibrium $\sigma^{*}$ is statically Pareto inefficient for any initial status quo $g_{0} \in \mathcal{I}$.

The key condition of Proposition 6 is $\theta_{A 2}<\theta_{i 1}<\theta_{B 2}$ for some $i \in\{A, B\}$. This has a natural interpretation, indicating that future polarization between the two parties must be greater than intertemporal preference variation for at least one party. Note that this occurs when party $A$ 's ideal is decreasing, or party $B$ 's ideal is increasing, implying that static Pareto inefficiency can arise when the ideal levels are increasing, decreasing or divergent. Since the proposition does not rule out $\theta_{A 1}=\theta_{B 1}$, static Pareto inefficiency can arise even in the absence of first-period conflict between the two parties.

Figure 3 provides an example of static inefficiency. The parameters used satisfy the conditions in Proposition 6. Specifically, the ideal levels of the two parties diverge, that is, $\theta_{A 2}<\theta_{A 1}<\theta_{B 1}<\theta_{B 2}$. The figure plots equilibrium public good spending in period 1 proposed by each party for initial status quo $g_{0} \in[0,2]$. What the figure shows is that unless $g_{0} \in\left[\theta_{A 1}, \theta_{B 1}\right]$, we have $\gamma_{i 1}^{*}\left(g_{0}\right) \notin\left[\theta_{A 1}, \theta_{B 1}\right]$ for at least one of the parties, that is, the equilibrium is statically Pareto inefficient.

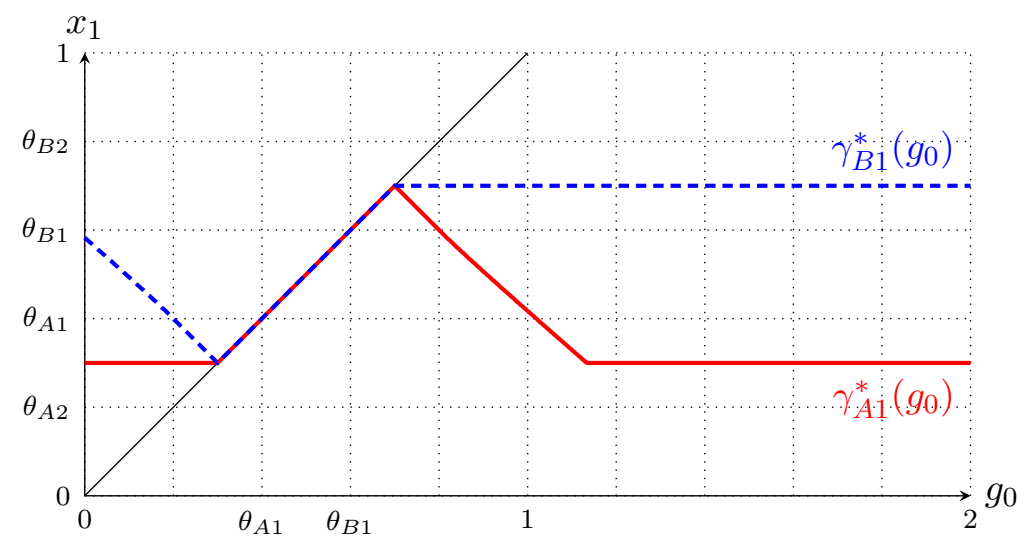

Figure 3: Period-1 equilibrium strategies when all spending is mandatory

$$
\boldsymbol{\theta}_{A}=(0.4,0.2), \boldsymbol{\theta}_{B}=(0.6,0.8), p_{A}=p_{B}=\frac{1}{2}, \delta=1, u_{i t}\left(x_{t}\right)=-\left(x_{t}-\theta_{i t}\right)^{2}
$$

The reason for static inefficiency is the dual role of $g_{1}$ : it is the spending in period 1 but it also determines the status quo in period 2. As an example, consider the case when party $A$ 's 
ideal is decreasing as in Figure 3. If party $A$ is the proposer in the first period, then it has an incentive to propose spending close to its period-1 ideal, but since period- 1 spending is the status quo for period 2, it also has an incentive to propose spending lower than its period-1 ideal. When party $B$ 's acceptance constraint is not binding, party $A$ proposes spending that is a weighted average of $\theta_{A 1}$ and $\theta_{A 2}$, giving rise to static inefficiency.

\section{Mandatory and discretionary combined}

We have seen that discretionary or mandatory programs in isolation typically lead to dynamic inefficiency. A natural question is whether the flexibility afforded by a combination of the two achieves dynamic efficiency. We now address this question by considering the case in which parties can endogenously choose the amount allocated to each of these programs. We begin by showing that when discretionary spending can only be positive, that is, $Z=\mathbb{R}_{+} \times \mathbb{R}_{+}$, we obtain dynamic efficiency under certain conditions.

Proposition 7. Under a budgetary institution that allows positive discretionary and mandatory spending, if utilities have increasing marginal returns and parties' ideals are decreasing, then every equilibrium is dynamically Pareto efficient for any initial status quo $g_{0} \in \mathbb{R}_{+}$.

We present the proof of Proposition 7 in the main text since it is instructive. First, consider the following alternative way of writing the social planner's dynamic problem:

$$
\begin{aligned}
\max _{\left(x_{1}, x_{A 2}, x_{B 2}\right) \in \mathbb{R}_{+}^{3}} & u_{i 1}\left(x_{1}\right)+\delta\left[p_{A} u_{i 2}\left(x_{A 2}\right)+p_{B} u_{i 2}\left(x_{B 2}\right)\right] \\
\text { s.t. } & u_{j 1}\left(x_{1}\right)+\delta\left[p_{A} u_{j 2}\left(x_{A 2}\right)+p_{B} u_{j 2}\left(x_{B 2}\right)\right] \geq \bar{U}
\end{aligned}
$$

for some $\bar{U} \in \mathbb{R}, i, j \in\{A, B\}$ and $i \neq j$. The difference between the original social planner's problem (DSP) and the modified social planner's problem (DSP') is that in the modified problem, the social planner is allowed to choose a distribution of allocations in period 2 . Since the utility functions are concave, it is not optimal for the social planner to randomize and therefore the solution to (DSP) is also the solution to (DSP'). To state this result formally, we denote the solution to (DSP) given $\bar{U} \in \mathbb{R}$ by $\mathbf{x}^{*}(\bar{U})=\left(x_{1}^{*}(\bar{U}), x_{2}^{*}(\bar{U})\right)$.

Lemma 3. The solution to the modified social planner's problem (DSP') is $x_{1}=x_{1}^{*}(\bar{U})$ and $x_{A 2}=x_{B 2}=x_{2}^{*}(\bar{U})$. 
Now fix the initial status quo $g_{0}$. Denote $f_{j}\left(g_{0}\right)$ as responder $j$ 's status quo payoff. That is $f_{j}\left(g_{0}\right)=u_{j 1}\left(g_{0}\right)+\delta V_{j}\left(g_{0} ; \sigma_{2}^{*}\right)$ with $\sigma_{2}^{*}$ given in Proposition 4 . The next result says that the equilibrium mandatory spending in period 1 is the dynamically Pareto efficient level of spending for period 2 corresponding to $\bar{U}$, and the sum of the equilibrium mandatory and discretionary spending is the dynamically Pareto efficient level of spending for period 1 corresponding to $\bar{U}$, where $\bar{U}$ is responder $j$ 's status quo payoff.

Lemma 4. Under a budgetary institution that allows positive discretionary and mandatory spending, if utilities have increasing marginal returns and parties' ideals are decreasing, then for any equilibrium $\sigma^{*}$, given initial status quo $g_{0}$, the equilibrium proposal strategy for party $i$ in period 1 satisfies $\gamma_{i 1}^{*}\left(g_{0}\right)=x_{2}^{*}(\bar{U})$ and $\kappa_{i 1}^{*}\left(g_{0}\right)=x_{1}^{*}(\bar{U})-x_{2}^{*}(\bar{U})$, where $\bar{U}=f_{j}\left(g_{0}\right)$.

Proof. If party $i$ is the proposer in period 1, then party $i$ 's equilibrium proposal strategy $\left(\kappa_{i 1}^{*}\left(g_{0}\right), \gamma_{i 1}^{*}\left(g_{0}\right)\right)$ is a solution to

$$
\begin{aligned}
\max _{\left(k_{1}, g_{1}\right) \in \mathbb{R}_{+}^{2}} & u_{i 1}\left(k_{1}+g_{1}\right)+\delta V_{i}\left(g_{1} ; \sigma_{2}^{*}\right) \\
\text { s.t. } & u_{j 1}\left(k_{1}+g_{1}\right)+\delta V_{j}\left(g_{1} ; \sigma_{2}^{*}\right) \geq u_{j 1}\left(g_{0}\right)+\delta V_{j}\left(g_{0} ; \sigma_{2}^{*}\right),
\end{aligned}
$$

where

$$
V_{i}\left(g ; \sigma_{2}^{*}\right)=p_{A} u_{i 2}\left(\kappa_{A 2}^{*}(g)+\gamma_{A 2}^{*}(g)\right)+p_{B} u_{i 2}\left(\kappa_{B 2}^{*}(g)+\gamma_{B 2}^{*}(g)\right) .
$$

For notational simplicity we write $x_{1}^{*}$ and $x_{2}^{*}$ instead of $x_{1}^{*}(\bar{U})$ and $x_{2}^{*}(\bar{U})$. We first show that $\left(x_{1}^{*}-x_{2}^{*}, x_{2}^{*}\right)$ is in the feasible set for $\left(P_{1}\right)$. As shown in Lemma A1 in the Appendix, if the parties' ideals are decreasing and utilities have increasing marginal returns, any Pareto efficient allocation is decreasing. This implies $x_{1}^{*}>x_{2}^{*}$, and thus $\left(x_{1}^{*}-x_{2}^{*}, x_{2}^{*}\right) \in \mathbb{R}_{+}^{2}$ is feasible.

We next show that if $\gamma_{i 1}^{*}\left(g_{0}\right)=x_{2}^{*}$ and $\kappa_{i 1}^{*}\left(g_{0}\right)=x_{1}^{*}-x_{2}^{*}$, then the induced equilibrium allocation is $x_{1}^{*}$ in period 1 and $x_{2}^{*}$ in period 2. It is straightforward to see $x_{1}^{\sigma^{*}}\left(g_{0}\right)=\gamma_{i 1}^{*}\left(g_{0}\right)+$ $\kappa_{i 1}^{*}\left(g_{0}\right)=x_{1}^{*}$. To see that $x_{2}^{\sigma^{*}}\left(g_{0}\right)=x_{2}^{*}$, first note that by Proposition 2 part $1, x_{2}^{*} \in\left[\theta_{A 2}, \theta_{B 2}\right]$. Proposition 4 part 2 then implies that $\kappa_{A 2}^{*}\left(x_{2}^{*}\right)+\gamma_{A 2}^{*}\left(x_{2}^{*}\right)=\kappa_{B 2}^{*}\left(x_{2}^{*}\right)+\gamma_{B 2}^{*}\left(x_{2}^{*}\right)=x_{2}^{*}$.

Finally, we show that $\left(x_{1}^{*}-x_{2}^{*}, x_{2}^{*}\right)$ is the maximizer of $\left(P_{1}\right)$. Suppose not. Then proposing $\left(\kappa_{i 1}^{*}\left(g_{0}\right), \gamma_{i 1}^{*}\left(g_{0}\right)\right)$ is better than proposing $\left(x_{1}^{*}-x_{2}^{*}, x_{2}^{*}\right)$. That is, proposing $\left(\kappa_{i 1}^{*}\left(g_{0}\right), \gamma_{i 1}^{*}\left(g_{0}\right)\right)$ gives proposer $i$ a higher dynamic payoff while giving the responder $j$ a dynamic payoff at 
least as high as $f_{j}\left(g_{0}\right)$. Hence, if $\left(\kappa_{i 1}^{*}\left(g_{0}\right), \gamma_{i 1}^{*}\left(g_{0}\right)\right) \neq\left(x_{1}^{*}-x_{2}^{*}, x_{2}^{*}\right)$, then the allocation with $x_{1}=\gamma_{i 1}^{*}\left(g_{0}\right)+\kappa_{i 1}^{*}\left(g_{0}\right), x_{A 2}=\kappa_{A 2}^{*}\left(\gamma_{i 1}^{*}\left(g_{0}\right)\right)+\gamma_{A 2}^{*}\left(\gamma_{i 1}^{*}\left(g_{0}\right)\right), x_{B 2}=\kappa_{B 2}^{*}\left(\gamma_{i 1}^{*}\left(g_{0}\right)\right)+\gamma_{B 2}^{*}\left(\gamma_{i 1}^{*}\left(g_{0}\right)\right)$ does better than $x_{1}=x_{1}^{*}$ and $x_{A 2}=x_{B 2}=x_{2}^{*}$ in (DSP'), which contradicts Lemma 3.

By Lemma 4, for status quo $g_{0}$ and period-1 proposer $i \in\{A, B\}$, the equilibrium outcome is dynamically Pareto efficient since $x_{1}^{\sigma^{*}}\left(g_{0}\right)=\gamma_{i 1}^{*}\left(g_{0}\right)+\kappa_{i 1}^{*}\left(g_{0}\right)=x_{1}^{*}(\bar{U})$ and $x_{2}^{\sigma^{*}}\left(g_{0}\right)=$ $\gamma_{i 1}^{*}\left(g_{0}\right)=x_{2}^{*}(\bar{U})$ where $\bar{U}=f_{j}\left(g^{0}\right)$. Hence $\sigma^{*}$ is a dynamically Pareto efficient equilibrium for any $g_{0} \in \mathbb{R}_{+}$. This completes the proof of Proposition 7 .

If the parties' ideals are increasing, and only positive discretionary spending is allowed together with mandatory spending, in general we do not obtain dynamic efficiency. This is because with increasing ideals we need discretionary spending in period 1 to be $x_{1}^{*}-x_{2}^{*}<0$ to achieve efficiency, which is not feasible. This suggests however that allowing negative discretionary spending restores dynamic Pareto efficiency, and indeed we have this result.

Proposition 8. Under a budgetary institution that allows positive and negative discretionary spending and positive mandatory spending, that is, $Z=\left\{\left(k_{t}, g_{t}\right) \in \mathbb{R} \times \mathbb{R}_{+} \mid k_{t}+g_{t} \geq 0\right\}$, every equilibrium $\sigma^{*}$ is dynamically Pareto efficient for any initial status quo $g_{0} \in \mathbb{R}_{+}$.

We omit the proof of Proposition 8 since it is a slight modification of the proof of Proposition 7. Negative discretionary spending implies that the total spending in the current period is lower than the status quo spending in the next period. As such, we can regard temporary cuts in mandatory spending as negative discretionary spending. Figure 4 below provides an example of equilibrium allocations for budgetary institutions that allow for mandatory spending programs, either in isolation or in combination with discretionary spending. The parameters used are the same as in Figure 3.

In Figure 4 the dashed blue line illustrates the set of dynamically Pareto efficient allocations, and the solid red line illustrates the set of equilibrium allocations - an initial status quo and a sequence of proposers induces an equilibrium allocation in the set. As shown in panels $4 \mathrm{a}$ and 4b, when mandatory spending and only positive discretionary spending are allowed, for some status quos and some realization of proposers, equilibrium allocations do not coincide with any dynamically Pareto efficient allocation. By contrast, when both positive 


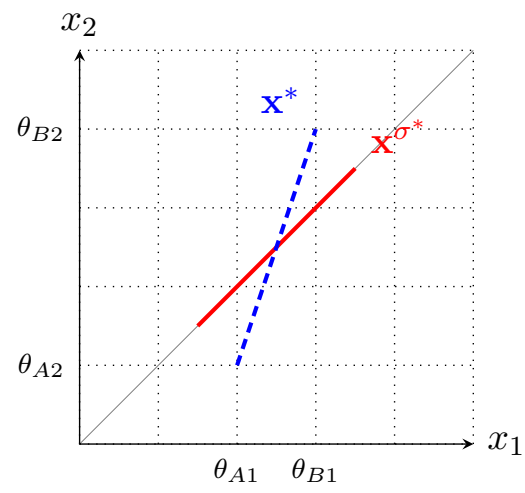

(a) Mandatory only

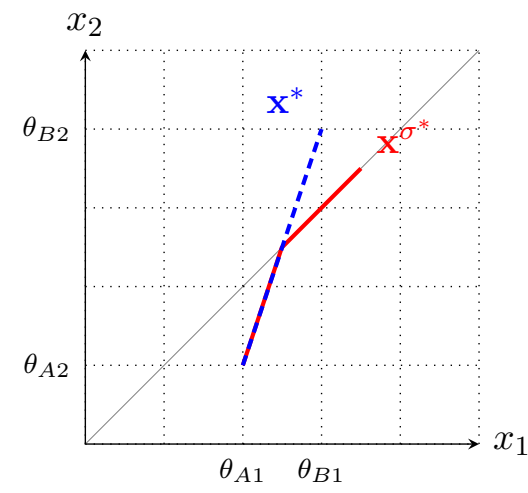

(b) Mandatory and positive only discretionary

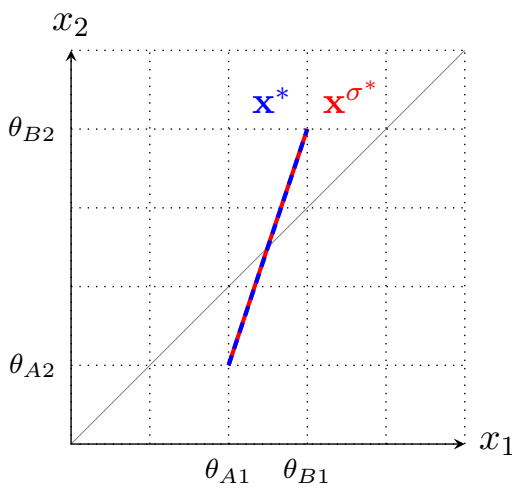

(c) Mandatory and positive or negative discretionary

Figure 4: Equilibrium allocations under different budgetary institutions

$$
\boldsymbol{\theta}_{A}=(0.4,0.2), \boldsymbol{\theta}_{B}=(0.6,0.8), p_{A}=p_{B}=\frac{1}{2}, \delta=1, u_{i t}\left(x_{t}\right)=-\left(x_{t}-\theta_{i t}\right)^{2}
$$

and negative discretionary spending are allowed, for any status quo and any realization of proposers, the equilibrium allocation coincides with a dynamically Pareto efficient allocation.

The reason the combination of mandatory and discretionary spending achieves dynamic Pareto efficiency is that the proposer in the first period can perfectly tailor the spending in that period to first-period preferences, and independently choose the next period's status quo. Although the first-period proposer cannot specify the whole sequence of allocations, neither gridlock nor political risk arises in equilibrium because spending fluctuates with preferences and the chosen second-period status quo is maintained regardless of who the proposer is. Allowing positive and negative discretionary spending together with mandatory spending provides sufficient flexibility to achieve this.

When there are more than two periods or preferences evolve stochastically, however, simply combining mandatory and discretionary spending no longer allows the proposer to perfectly tailor the spending in the current period to the preferences in that period and independently choose the status quos for all future periods. Therefore efficiency can no longer be achieved. In order to achieve efficiency, more flexibility is needed. We illustrate next that if preferences evolve deterministically, then sunset provisions with appropriately chosen expiration dates achieve efficiency with more than two periods. 
Sunset provisions Consider the following three-period extension with sunset provisions. A proposal in period $t$ is $z_{t}=\left(k_{t}, s_{t}, g_{t}\right)$. As before, $k_{t}$ is discretionary spending for period $t$ and $g_{t}$ is mandatory spending. The new component is sunset provision $s_{t}$, which is spending that applies in periods $t$ and $t+1$ and expires thereafter. If $z_{t}$ is accepted, then the spending in period $t$ is $x_{t}=k_{t}+s_{t}+g_{t}$ and the status quo in period $t+1$ is $\left(s_{t}, g_{t}\right)$. If $z_{t}$ is rejected, then the spending in period $t$ is $x_{t}=s_{t-1}+g_{t-1}$ and the status quo in period $t+1$ is $\left(0, g_{t-1}\right)$.

Note that an accepted proposal $z_{1}=\left(k_{1}, s_{1}, g_{1}\right)$ determines spending in the first period $x_{1}=k_{1}+s_{1}+g_{1}$, the status quo spending in the second period $x_{2}=s_{1}+g_{1}$ and the status quo spending in the third period $x_{3}=g_{1}$. Therefore, sunset provisions in combination with mandatory and discretionary spending allow the proposer to choose today's spending independently of future status quos, and choose future status quos independently of each other (this is not possible with only mandatory and discretionary spending). In the first period the proposer can tailor the status quo spending for each future period such that the future proposer in that period has no incentive to change it. Following the intuition of Lemma 4, the equilibrium first-period proposal $z_{1}=\left(k_{1}, s_{1}, g_{1}\right)$ induces an allocation $\mathbf{x}=\left(k_{1}+s_{1}+g_{1}, s_{1}+g_{1}, g_{1}\right)$, which is dynamically Pareto efficient and remains unchanged in later periods. This avoids gridlock by allowing spending to fluctuate with the evolving preferences, and eliminates political risk by ensuring that the spending levels do not depend on the identity of future proposers. This result holds more generally. Specifically, beyond three periods, multiple sunset provisions with different expiration dates allow the proposer to choose status quo spending for each future period independently and therefore provide sufficient flexibility required for dynamic efficiency. ${ }^{33}$

Stochastically evolving preferences require further flexibility for efficiency to be achieved. In the next section we consider a model with arbitrary time horizon and stochastic preferences and show that state-contingent mandatory spending provides such flexibility.

\footnotetext{
${ }^{33}$ Note that with sunset provisions, flexibility is introduced by adding dimensions to the policy space. It is possible that these additional policy instruments are not available, in which case one might ask if reducing flexibility by placing bounds on mandatory and discretionary programs might improve efficiency. That is, we might restrict the set of policies to $Z=[a, b] \times[c, d] \subseteq \mathbb{R} \times \mathbb{R}_{+}$and ask what values of $(a, b, c, d)$ are optimal. We leave this inquiry to future work.
} 


\section{State-contingent mandatory spending}

Consider a richer environment in which parties bargain in $T \geq 2$ periods and preferences are stochastic in each period reflecting uncertainties in the economy. ${ }^{34}$ The economic state (henceforth we refer to the economic state as simply the state) in each period $t$ is $s_{t} \in S$ where $S$ is a finite set of $n=|S|$ possible states. We assume the distribution of states has full support in every period, but do not require the distribution to be the same across periods. ${ }^{35}$ The utility of party $i$ in period $t$ when the spending is $x$ and the state is $s$ is $u_{i}(x, s)$. As before, we assume $u_{i}(x, s)$ is twice continuously differentiable and strictly concave in $x$. Further, $u_{i}(x, s)$ attains a maximum at $\theta_{i s}$ and we assume $0<\theta_{A s}<\theta_{B s}$ for all $s \in S$. The state is drawn at the beginning of each period before a proposal is made. We denote the probability that party $i$ proposes in period $t$ by $p_{i t} \in(0,1)$, which can depend on $t$ arbitrarily. In this setting, we consider a budgetary institution that allows state-contingent mandatory spending. As discussed in the Introduction, these state-contingent programs have been used historically, and are still in use.

A proposal in period $t$ is a spending rule $g_{t}: S \rightarrow \mathbb{R}_{+}$where $g_{t}(s)$ is the level of public good spending proposed to be allocated to the mandatory program in state $s$. If the responding party agrees to the proposal, the allocation implemented in period $t$ is $g_{t}\left(s_{t}\right)$; otherwise the allocation in period $t$ is given by the status quo spending rule $g_{t-1}\left(s_{t}\right)$. In this environment, a strategy for party $i$ in period $t$ is $\sigma_{i t}=\left(\gamma_{i t}, \alpha_{i t}\right)$. Let $\mathcal{M}$ be the space of all functions from $S$ to $\mathbb{R}_{+}$. Then $\gamma_{i t}: \mathcal{M} \times S \rightarrow \mathcal{M}$ is a proposal strategy for party $i$ in period $t$ and $\alpha_{i t}: \mathcal{M} \times S \times \mathcal{M} \rightarrow\{0,1\}$ is an acceptance strategy for party $i$ in period $t$. A strategy for party $i$ is $\sigma_{i}=\left(\sigma_{i 1}, \ldots, \sigma_{i T}\right)$ and a profile of strategies is $\sigma=\left(\sigma_{1}, \sigma_{2}\right)$.

With stochastic preferences the social planner chooses an allocation rule $x_{t}: S \rightarrow \mathbb{R}_{+}$for all $t \in\{1, \ldots, T\}$ to maximize the expected payoff of one of the parties subject to providing the other party with a minimum expected dynamic payoff. Formally, a dynamically Pareto

\footnotetext{
${ }^{34}$ Note that $T$ can be finite or infinite. In the case of infinite horizon we assume $\delta \in(0,1)$ so that dynamic utilities are well-defined.

${ }^{35}$ We assume full support in this section for expositional simplicity, but Proposition 10 below still holds in an extension in which the distribution of states has different supports in different periods.
} 
efficient allocation rule solves the following maximization problem:

$$
\begin{aligned}
\max _{\left\{x_{t}: S \rightarrow \mathbb{R}_{+}\right\}_{t=1}^{T}} & \sum_{t=1}^{T} \delta^{t-1} \mathbb{E}_{s_{t}}\left[u_{i}\left(x_{t}\left(s_{t}\right), s_{t}\right)\right] \\
\text { s.t. } & \sum_{t=1}^{T} \delta^{t-1} \mathbb{E}_{s_{t}}\left[u_{j}\left(x_{t}\left(s_{t}\right), s_{t}\right)\right] \geq \bar{U},
\end{aligned}
$$

for some $\bar{U} \in \mathbb{R}, i, j \in\{A, B\}$ and $i \neq j$. We denote the solution to (DSP-S) by the sequence of functions $\mathbf{x}^{*}=\left\{x_{t}^{*}\right\}_{t=1}^{T}$. The next proposition characterizes dynamically Pareto efficient allocation rules, analogous to Proposition 2.

Proposition 9. Any dynamically Pareto efficient allocation rule satisfies:

1. For any $t$ and $t^{\prime}, x_{t}^{*}=x_{t^{\prime}}^{*}$.

2. For all $s \in S$ and all $t$, either

$$
-\frac{u_{i}^{\prime}\left(x_{t}^{*}(s), s\right)}{u_{j}^{\prime}\left(x_{t}^{*}(s), s\right)}=\lambda^{*}
$$

for some $\lambda^{*}>0$, or $x_{t}^{*}(s)=\theta_{A s}$, or $x_{t}^{*}(s)=\theta_{B s}$.

Proposition 9 first says that the dynamically Pareto efficient allocation rule is independent of time, i.e., the same allocation rule is used each period. The second part of the proposition says that the dynamically Pareto efficient allocation rule either satisfies the condition that the ratio of the parties' marginal utilities is constant across states, or is one party's ideal in each state.

We next define a dynamically Pareto efficient equilibrium and show that dynamic efficiency is obtained by state-contingent mandatory spending. Define recursively $x_{t}^{\sigma^{*}}\left(g_{0}\right)$ for $t \in\{1, \ldots, T\}$ by $x_{1}^{\sigma^{*}}\left(g_{0}\right)=\gamma_{i 1}^{*}\left(g_{0}, s_{1}\right)$ for some $i \in\{A, B\}$ and some $s_{1} \in S$, and $x_{t}^{\sigma^{*}}\left(g_{0}\right)=\gamma_{i t}^{*}\left(x_{t-1}^{\sigma^{*}}\left(g_{0}\right), s_{t}\right)$ for some $i \in\{A, B\}$ and some $s_{t} \in S$ and $t \in\{2, \ldots, T\}$. An equilibrium allocation rule for $\sigma^{*}$ given initial status quo $g_{0}$ is a possible realization of a spending rule for each period, $\mathbf{x}^{\sigma^{*}}\left(g_{0}\right)=\left\{x_{t}^{\sigma^{*}}\left(g_{0}\right)\right\}_{t=1}^{T}$. The random determination of proposers and states in each period induces a probability distribution over allocation rules given an equilibrium $\sigma^{*}$. Thus any element in the support of this distribution is an equilibrium allocation rule for $\sigma^{*}$. 
Definition 2. An equilibrium $\sigma^{*}$ is a dynamically Pareto efficient equilibrium given initial status quo $g_{0} \in \mathcal{M}$ if and only if every equilibrium allocation rule $\mathbf{x}^{\sigma^{*}}\left(g_{0}\right)$ for $\sigma^{*}$ given initial status quo $g_{0}$ is dynamically Pareto efficient.

Proposition 10. Under state-contingent mandatory spending, an equilibrium is either dynamically Pareto efficient for any initial status quo $g_{0} \in \mathcal{M}$ or is outcome-equivalent to a dynamically Pareto efficient equilibrium.

The result in Proposition 10 is in stark contrast to the inefficiency results for mandatory spending given in Propositions 5 and 6. Recall that dynamic efficiency fails in the model with evolving (deterministic) preferences and fixed mandatory spending because the proposer in period 1 cannot specify spending in the current period separately from the status quo for the next period. Proposition 10 can be understood in an analogous way to the efficiency result with discretionary and mandatory spending combined. In the first period the proposer can tailor the status quo for each state such that any acceptable proposal other than the status quo makes the future proposer worse off, giving the future proposer no incentive to change it. This avoids gridlock by allowing spending to fluctuate with the economic state, and eliminates political risk by ensuring that the spending levels do not depend on the identity of future proposers. ${ }^{36}$ Thus, even though the identity of the proposer is not contractible, in equilibrium inefficiency arising from proposer uncertainty is eliminated through the status quo. State-contingent mandatory spending therefore overcomes inefficiency due to two kinds of uncertainty - uncertainty about states, which is contractible, and uncertainty about the proposer identity, which is not contractible.

\section{Conclusion}

In this paper we demonstrate that discretionary only and mandatory only budgetary institutions typically result in dynamic inefficiency, and may result in static inefficiency in the

\footnotetext{
${ }^{36}$ We can regard the two-period model analyzed in the previous sections as a special case of an extension of this section's model in which the distribution of states has different supports in different periods with a degenerate distribution in each period. The state-contingent mandatory programs achieve dynamic efficiency by allowing the total spending in period 1 to be different from the status quo spending in period 2 , which can also be achieved by a combination of mandatory and discretionary programs in the two-period setting.
} 
case of mandatory only budgetary institutions. However, we show that bargaining achieves dynamic Pareto efficiency in increasingly complex environments when flexibility is introduced either through the endogenous combination of mandatory and discretionary programs, or through sunset provisions, or through a state-contingent mandatory program. We show that these budgetary institutions eliminate political risk and gridlock by allowing the proposer to choose status quos that are not changed by future proposers because they fully account for fluctuations in preferences.

We have considered mandatory spending programs that are fully state-contingent, but it is possible that factors influencing parties' preferences, such as the mood of the electorate, cannot be contracted on. In this case it seems there is room for inefficiency even with mandatory spending that depends on a contractible state. It is possible that further flexibility with discretionary spending may be helpful. Such combinations are observed in practice; for example, in the United States, unemployment insurance is provided through both state-contingent mandatory programs and discretionary programs. ${ }^{37}$ However, including discretionary spending may leave more room for political risk. We leave for future work exploring efficiency implications of discretionary and mandatory spending when a part of the state may not be contracted on.

\section{References}

Acemoglu, D., Golosov, M. and Tsyvinski, A. (2008), 'Political economy of mechanisms', Econometrica 76(3), 619-641.

Acemoglu, D., Golosov, M. and Tsyvinski, A. (2011), 'Power fluctuations and political economy', Journal of Economic Theory 146(3), 1009-1041.

Aguiar, M. and Amador, M. (2011), 'Growth in the shadow of expropriation', Quarterly Journal of Economics 126(2), 651-697.

Alesina, A. and Perotti, R. (1995), 'The political economy of budget deficits', Staff Papers International Monetary Fund 42(1), 1-31.

Alesina, A. and Tabellini, G. (1990), 'A positive theory of fiscal deficits and government

\footnotetext{
${ }^{37}$ See Department of Labor Budget in Brief, United States Department of Labor (2015).
} 
debt', Review of Economic Studies 57(3), 403-414.

Anesi, V. and Seidmann, D. J. (2015), 'Bargaining in standing committees with an endogenous default', forthcoming in Review of Economic Studies .

Azzimonti, M. (2011), 'Barriers to investment in polarized societies', American Economic Review 101(5), 2182-2204.

Azzimonti, M., Battaglini, M. and Coate, S. (2015), 'The costs and benefits of balanced budget rules: Lessons from a political economy model of fiscal policy', Working Paper .

Bai, J. and Lagunoff, R. (2011), 'On the 'Faustian' dynamics of policy and political power', Review of Economic Studies 78(1), 17-48.

Baron, D. (1996), 'A dynamic theory of collective goods programs', American Political Science Review 90(2), 316-330.

Baron, D. and Bowen, T. R. (2014), 'Dynamic coalitions', Working paper .

Battaglini, M. and Coate, S. (2007), 'Inefficiency in legislative policy-making: A dynamic analysis', American Economic Review 97(1), 118-149.

Battaglini, M. and Palfrey, T. (2012), 'The dynamics of distributive politics', Economic Theory 49(3), 739-777.

Besley, T. and Coate, S. (1998), 'Sources of inefficiency in a representative democracy: A dynamic analysis', American Economic Review 88(1), 139-156.

Besley, T. and Coate, S. (2003), 'Centralized versus decentralized provision of local public goods: A political economy approach', Journal of Public Economics 87(12), 2611-2637.

Besley, T. and Smart, M. (2007), 'Fiscal restraints and voter welfare', Journal of Public Economics 91(3-4), 755-773.

Bierbrauer, F. J. and Boyer, P. (2014), 'Efficiency, welfare, and political competition', CESifo Working Paper Series 4814.

Bowen, T. R., Chen, Y. and Eraslan, H. (2014), 'Mandatory versus discretionary spending: The status quo effect', American Economic Review 104(10), 2941-2974.

Breasted, J. H. (1945), A History of Egypt, Charles Scribner's Sons, New York, NY.

Budget of the United States Government (2015), 'Budget of the United States Government, Fiscal year 2015, Historical Tables and Public Budget Database'. 
URL: http://www.gpo.gov/fdsys/browse/collectionGPO.action?collectionCode=BUDGET Caballero, R. J. and Yared, P. (2010), 'Future rent-seeking and current public savings', Journal of International Economics 82(2), 124-136.

Callander, S. and Krehbiel, K. (2014), 'Gridlock and delegationin a changing world', American Journal of Political Science 58(4), 819-834.

Canadian Minister of Justice (2014), 'Employment Insurance Act'.

URL: http://laws-lois.justice.gc.ca/pdf/e-5.6.pdf

Chen, Y. and Eraslan, H. (2014), 'Dynamic Agenda Setting', Working Paper .

Diermeier, D., Egorov, G. and Sonin, K. (2013), 'Endogenous property rights', National Bureau of Economic Research Working Paper Series 19734.

Diermeier, D. and Fong, P. (2011), 'Legislative bargaining with reconsideration', Quarterly Journal of Economics 126(2), 947-985.

Dixit, A., Grossman, G. M. and Gul, F. (2000), 'The dynamics of political compromise', Journal of Political Economy 108(3), 531-568.

Duggan, J. and Kalandrakis, T. (2012), 'Dynamic legislative policy making', Journal of Economic Theory 147(5), 1653-1688.

Dziuda, W. and Loeper, A. (2015), 'Dynamic collective choice with endogenous status quo', forthcoming in Journal of Political Economy .

Edlin, A. S. and Shannon, C. (1998), 'Strict monotonicity in comparative statics', Journal of Economic Theory 81(1), 201-219.

Forand, J. G. (2014), 'Two-party competition with persistent policies', Journal of Economic Theory 152, 64-91.

Halac, M. and Yared, P. (2014), 'Fiscal rules and discretion under persistent shocks', Econometrica 82(5), 1557-1614.

Hallerberg, M., Strauch, R. R. and von Hagen, J. (2009), Fiscal Governance in Europe, Cambridge University Press, Cambridge.

House Budget Committee (2014), 'The path to prosperity: Fiscal year 2015 budget resolution'.

URL: http://budget.house.gov/fy2015/ 
Kalandrakis, A. (2014), 'Pareto efficiency in the dynamic one-dimensional bargaining model', University of Rochester Wallis Institute of Political Economy Working Paper Series 66.

Kalandrakis, T. (2004), 'A three-player dynamic majoritarian bargaining game', Journal of Economic Theory 116(2), 294-322.

Kalandrakis, T. (2010), 'Minimum winning coalitions and endogenous status quo', International Journal of Game Theory 39(4), 617-643.

Krusell, P. and Ríos-Rull, J.-V. (1996), 'Vested interests in a positive theory of stagnation and growth', Review of Economic Studies 63(2), 301-329.

Levy, G. and Razin, R. (2013), 'Dynamic legislative decision making when interest groups control the agenda', Journal of Economic Theory 148(5), 1862-1890.

Lizzeri, A. and Persico, N. (2001), 'The provision of public goods under alternative electoral incentives', American Economic Review 91(1), 225-239.

Ma, Y. (2014), 'Curse or blessing? On the welfare consequences of divided government and policy gridlock', Working Paper .

Nicholson, W. and Needels, K. (2006), 'Unemployment insurance: Strengthening the relationship between theory and policy', Journal of Economic Perspectives 20(3), 47-70.

Nunnari, S. and Zapal, J. (2014), 'Dynamic elections and ideological polarization', Working Paper .

OECD (2012), 'International budget practices and procedures database'.

URL: http://www.oecd.org/gov/budgeting/

Persson, T. and Svensson, L. E. O. (1984), 'Time-consistent fiscal policy and government cash-flow', Journal of Monetary Economics 14(3), 365-374.

Persson, T. and Tabellini, G. (1996a), 'Federal fiscal constitutions: Risk sharing and moral hazard', Econometrica 64(3), 623-646.

Persson, T. and Tabellini, G. (1996b), 'Federal fiscal constitutions: Risk sharing and redistribution', Journal of Political Economy 104(5), 979-1009.

Piguillem, F. and Riboni, A. (2012), 'Dynamic bargaining over redistribution in legislatures', Working Paper .

Piguillem, F. and Riboni, A. (2015), 'Spending-biased legislators: Discipline through dis- 
agreement', Quarterly Journal of Economics 130(2), 901-949.

Riboni, A. and Ruge-Murcia, F. J. (2008), 'The dynamic (in)efficiency of monetary policy by committee', Journal of Money, Credit and Banking 40(5), 1001-1032.

Romer, T. and Rosenthal, H. (1978), 'Political resource allocation, controlled agendas, and the status quo', Public Choice 33(48), 27-43.

Romer, T. and Rosenthal, H. (1979), 'Bureaucrats versus voters: On the political economy of resource allocation by direct democracy', Quarterly Journal of Economics 93(4), 563-587.

Santiso, C. (2006), 'Budget institutions and fiscal responsibility: Parliaments and the political economy of the budget process', World Bank Institute Working Paper Series $\mathbf{3 5 8 6 3 .}$

Shah, A. (2007), Budgeting and Budgetary Institutions, The World Bank, Washington DC.

Stockman, D. R. (2001), 'Balanced-budget rules: Welfare loss and optimal policies', Review of Economic Dynamics 4(2), 438-459.

Takayama, A. (1974), Mathematical Economics, Dryden Press, Hinsdale, IL.

United States Department of Labor (2015), 'Fiscal year 2015 Department of Labor budget in brief'.

URL: http://www.dol.gov/dol/budget/2015/pdf/fy2015bib.pdf

U.S. House of Representatives, Office of the Legislative Counsel (2013), 'Federal-state extended unemployment compensation act of 1970'.

URL: http://legcounsel.house.gov/Comps/fseuca70.pdf

Van Weelden, R. (2013), 'Candidates, credibility, and re-election incentives', Review of Economic Studies 80(4), 1622-1651.

Yared, P. (2010), 'Politicians, taxes and debt', Review of Economic Studies 77(2), 806-840.

Zapal, J. (2011), 'Explicit and implicit status-quo determination in dynamic bargaining: Theory and application to FOMC directive', Working paper . 


\section{Appendix}

\section{A1 Pareto efficiency}

\section{A1.1 Proof of Proposition 1}

First, we show that if $x_{t}$ is statically Pareto efficient, then $x_{t} \in\left[\theta_{A t}, \theta_{B t}\right]$. Consider $x_{t} \notin\left[\theta_{A t}, \theta_{B t}\right]$. Then we can find $x_{t}^{\prime}$ in either $\left(x_{t}, \theta_{A t}\right)$ or $\left(\theta_{B t}, x_{t}\right)$ such that $u_{A t}\left(x_{t}^{\prime}\right)>u_{A t}\left(x_{t}\right)$ and $u_{B t}\left(x_{t}^{\prime}\right)>u_{B t}\left(x_{t}\right)$, and therefore $x_{t}$ is not a solution to (SSP).

Second, we show that if $\tilde{x}_{t} \in\left[\theta_{A t}, \theta_{B t}\right]$, then $\tilde{x}_{t}$ is statically Pareto efficient. Let $\bar{u}=$ $u_{j t}\left(\tilde{x}_{t}\right)$. Denote the solution to (SSP) as $\hat{x}_{t}(\bar{u})$. Since $u_{A t}^{\prime}\left(x_{t}\right)<0$ and $u_{B t}^{\prime}\left(x_{t}\right)>0$ for all $x_{t} \in\left(\theta_{A t}, \theta_{B t}\right)$, the solution to $(\mathrm{SSP})$ is $\hat{x}_{t}(\bar{u})=\tilde{x}_{t}$ for any $\tilde{x}_{t} \in\left[\theta_{A t}, \theta_{B t}\right]$.

\section{A1.2 Proof of Proposition 2}

We prove the result for a more general model with $T \geq 2$. Denote a sequence of allocations by $\mathbf{x}=\left\{x_{t}\right\}_{t=1}^{T}$ and party $i$ 's discounted dynamic payoff from $\mathbf{x}=\left\{x_{t}\right\}_{t=1}^{T}$ by $U_{i}(\mathbf{x})=$

$\sum_{t=1}^{T} \delta^{t-1} u_{i t}\left(x_{t}\right)$. We define a dynamically Pareto efficient allocation in the $T$-period problem as the solution to the following maximization problem

$$
\begin{aligned}
\max _{\mathbf{x} \in \mathbb{R}_{+}^{T}} & U_{i}(\mathbf{x}) \\
\text { s.t. } & U_{j}(\mathbf{x}) \geq \bar{U}
\end{aligned}
$$

for some $\bar{U} \in \mathbb{R}, i, j \in\{A, B\}$ and $i \neq j$. Denote the sequence of party $i$ 's static ideals by $\boldsymbol{\theta}_{i}=\left\{\theta_{i t}\right\}_{t=1}^{T}$ for all $i \in\{A, B\}$, and denote the solution to (DSP) as $\mathbf{x}^{*}=\left\{x_{t}^{*}\right\}_{t=1}^{T}$.

To prove part 1 , by way of contradiction, suppose $x_{t^{\prime}}^{*} \notin\left[\theta_{A t^{\prime}}, \theta_{B t^{\prime}}\right]$. By Proposition 1 there exists $\hat{x}_{t^{\prime}}$ such that $u_{i t^{\prime}}\left(\hat{x}_{t^{\prime}}\right) \geq u_{i t^{\prime}}\left(x_{t^{\prime}}^{*}\right)$ for all $i \in\{A, B\}$, and $u_{i t^{\prime}}\left(\hat{x}_{t^{\prime}}\right)>u_{i t^{\prime}}\left(x_{t^{\prime}}^{*}\right)$ for at least one $i \in\{A, B\}$. Now consider $\hat{\mathbf{x}}=\left\{\hat{x}_{t}\right\}_{t=1}^{T}$, with $\hat{x}_{t}=x_{t}^{*}$ for all $t \neq t^{\prime}$. Then $U_{i}(\hat{\mathbf{x}}) \geq U_{i}\left(\mathbf{x}^{*}\right)$ for all $i \in\{A, B\}$, and $U_{i}(\hat{\mathbf{x}})>U_{i}\left(\mathbf{x}^{*}\right)$ for at least one $i \in\{A, B\}$. Thus $\mathbf{x}^{*}$ is not dynamically Pareto efficient.

Next we prove part 2 by considering possible values of $\bar{U}$. Fix $i, j \in\{A, B\}$ with $i \neq j$. Since for any $\bar{U}>U_{j}\left(\boldsymbol{\theta}_{j}\right)$ the solution does not exist, we only need to consider $\bar{U} \leq U_{j}\left(\boldsymbol{\theta}_{j}\right)$.

For $\bar{U}=U_{j}\left(\boldsymbol{\theta}_{j}\right)$, the solution to (DSP) is $\mathbf{x}^{*}=\boldsymbol{\theta}_{j}$ and for any $\bar{U} \leq U_{j}\left(\boldsymbol{\theta}_{i}\right)$, the solution to 
(DSP) is $\mathbf{x}^{*}=\boldsymbol{\theta}_{i}$. What remains is to consider the case when $\bar{U} \in\left(U_{j}\left(\boldsymbol{\theta}_{i}\right), U_{j}\left(\boldsymbol{\theta}_{j}\right)\right)$. Suppose $\boldsymbol{\theta}_{A}=\boldsymbol{\theta}_{B}$, then $U_{j}\left(\boldsymbol{\theta}_{i}\right)=U_{j}\left(\boldsymbol{\theta}_{j}\right)$, which implies $\mathbf{x}^{*}=\boldsymbol{\theta}_{i}$. So consider $\boldsymbol{\theta}_{A} \neq \boldsymbol{\theta}_{B}$.

For the rest of the proof, suppose $\bar{U} \in\left(U_{j}\left(\boldsymbol{\theta}_{i}\right), U_{j}\left(\boldsymbol{\theta}_{j}\right)\right)$. Consider a relaxed version of (DSP) with the constraint $\mathbf{x} \in \mathbb{R}_{+}^{T}$ dropped. Denote the solution to the relaxed problem as $\hat{\mathbf{x}}=\left\{\hat{x}_{t}\right\}_{t=1}^{T}$. Similar to the proof of part 1 , if $\hat{x}_{t} \notin\left[\theta_{A t}, \theta_{B t}\right]$, then both parties can be made strictly better off in period $t$. Thus if $\hat{\mathbf{x}}$ is a solution to the relaxed problem, then $\hat{x}_{t} \in\left[\theta_{A t}, \theta_{B t}\right]$ for all $t$. It follows that the solution to the relaxed problem is the same as the solution to (DSP). The Lagrangian of the relaxed problem is

$$
L(\mathbf{x}, \lambda)=U_{i}(\mathbf{x})-\lambda\left[-U_{j}(\mathbf{x})+\bar{U}\right] .
$$

By Takayama (1974, Theorem 1.D.4), if the Jacobian of the constraint has rank 1 then the solution to (DSP) satisfies

$$
\frac{\partial L(\hat{\mathbf{x}}, \hat{\lambda})}{\partial x_{t}}=\delta^{t-1} u_{i t}^{\prime}\left(\hat{x}_{t}\right)+\hat{\lambda} \delta^{t-1} u_{j t}^{\prime}\left(\hat{x}_{t}\right)=0
$$

with $\hat{\lambda} \geq 0$. The Jacobian is

$$
\left[\left(\delta^{t-1} \frac{\partial u_{j t}\left(\hat{x}_{t}\right)}{\partial x_{t}}\right)_{t=1}^{T}\right]
$$

and it has rank 1 if there exists $t^{\prime}$ such that $\hat{x}_{t^{\prime}} \neq \theta_{j t^{\prime}}$, which we show next. Suppose $\hat{\mathbf{x}}=\boldsymbol{\theta}_{j}$. This implies $U_{j}(\hat{\mathbf{x}})>\bar{U}$. Because there exists $t^{\prime}$ such that $\theta_{i t^{\prime}} \neq \theta_{j t^{\prime}}$, we can find $\alpha \in(0,1)$ such that $\mathbf{x}=\left\{x_{t}\right\}_{t=1}^{T}$ with $x_{t^{\prime}}=\alpha \theta_{i t^{\prime}}+(1-\alpha) \theta_{j t^{\prime}}$ and $x_{t}=\hat{x}_{t}$ for all $t \neq t^{\prime}$ satisfies the constraints in (DSP) and strictly increases the value of the objective function relative to $\hat{\mathbf{x}}$.

If $\hat{\lambda}=0$, we obtain $\hat{\mathbf{x}}=\boldsymbol{\theta}_{i}$, violating the $U_{j}(\mathbf{x}) \geq \bar{U}$ constraint, and hence $\hat{\lambda}>0$.

\section{A1.3 Proof of Lemma 1}

Since $\mathbf{x}$ and $\tilde{\mathbf{x}}$ are dynamically Pareto efficient, by Proposition 2 part $1, x_{t} \in\left[\theta_{A t}, \theta_{B t}\right]$ and $\tilde{x}_{t} \in\left[\theta_{A t}, \theta_{B t}\right]$ for all $t$. There are three possible cases.

Case (i): $x_{t^{\prime}}=\tilde{x}_{t^{\prime}}=\theta_{A t^{\prime}}$. By Proposition 2, part 2 either $\mathbf{x}=\boldsymbol{\theta}_{A}$, or $\mathbf{x}=\boldsymbol{\theta}_{B}$, or $u_{A t^{\prime}}^{\prime}\left(x_{t^{\prime}}\right)+\lambda^{*} u_{B t^{\prime}}^{\prime}\left(x_{t^{\prime}}\right)=0$ for some $\lambda^{*}>0$. Since $\theta_{A t} \neq \theta_{B t}$ for any $t, x_{t^{\prime}}=\theta_{A t^{\prime}}$ implies $\mathbf{x} \neq \boldsymbol{\theta}_{B}$. Next note that $u_{A t}^{\prime}\left(\theta_{A t}\right)=0$ for all $t$ and $u_{B t}^{\prime}\left(\theta_{A t}\right) \neq 0$ for any $t$, hence $u_{A t^{\prime}}^{\prime}\left(x_{t^{\prime}}\right)+\lambda^{*} u_{B t^{\prime}}^{\prime}\left(x_{t^{\prime}}\right) \neq$ 0 for any $\lambda^{*}>0$. Thus, it must be that $\mathbf{x}=\boldsymbol{\theta}_{A}$. A similar argument shows that $\tilde{\mathbf{x}}=\boldsymbol{\theta}_{A}$, 
proving that $\mathbf{x}=\tilde{\mathbf{x}}$.

Case (ii): $x_{t^{\prime}}=\tilde{x}_{t^{\prime}}=\theta_{B t^{\prime}}$. Analogous to case (i), if $x_{t^{\prime}}=\tilde{x}_{t^{\prime}}=\theta_{B t^{\prime}}$, then $\mathbf{x}=\tilde{\mathbf{x}}=\boldsymbol{\theta}_{B}$.

Case (iii): $x_{t^{\prime}}=\tilde{x}_{t^{\prime}} \in\left(\theta_{A t^{\prime}}, \theta_{B t^{\prime}}\right)$. Note that $\mathbf{x} \neq \boldsymbol{\theta}_{i}$ for any $i$. By Proposition 2, part 2 it must be that $u_{A t^{\prime}}^{\prime}\left(x_{t^{\prime}}\right)+\lambda^{*} u_{B t^{\prime}}^{\prime}\left(x_{t^{\prime}}\right)=0$ for some $\lambda^{*}>0$. This implies $-\frac{u_{A A^{\prime}}^{\prime}\left(x_{t^{\prime}}\right)}{u_{B t^{\prime}}^{\prime}\left(x_{t^{\prime}}\right)}=\lambda^{*}$. Similarly, $u_{A t^{\prime}}^{\prime}\left(\tilde{x}_{t^{\prime}}\right)+\tilde{\lambda}^{*} u_{B t^{\prime}}^{\prime}\left(\tilde{x}_{t^{\prime}}\right)=0$ for some $\tilde{\lambda}^{*}>0$, implying $-\frac{u_{A t^{\prime}}^{\prime}\left(\tilde{x}_{t^{\prime}}\right)}{u_{B t^{\prime}}^{\prime}\left(\tilde{x}_{t^{\prime}}\right)}=\tilde{\lambda}^{*}$. Since $x_{t^{\prime}}=\tilde{x}_{t^{\prime}}$ it follows that $\lambda^{*}=\tilde{\lambda}^{*}$. Then by Proposition 2 part $2,-\frac{u_{A t}^{\prime}\left(x_{t}\right)}{u_{B t}^{\prime}\left(x_{t}\right)}=-\frac{u_{A t}^{\prime}\left(\tilde{x}_{t}\right)}{u_{B t}^{\prime}\left(\tilde{x}_{t}\right)}=\lambda^{*}$ for all $t$. To prove $x_{t}=\tilde{x}_{t}$ for all $t$, it remains to show that $-\frac{u_{A t}^{\prime}(x)}{u_{B t}^{\prime}(x)}=\lambda^{*}$ has a unique solution for any $\lambda^{*}>0$. To see this, first note that $\mathbf{x} \neq \boldsymbol{\theta}_{i}$ for any $i$ implies $x_{t} \in\left(\theta_{A t}, \theta_{B t}\right)$ for all $t$ because otherwise, $x_{t}=\theta_{i t}$ for some $i$ and some $t$, and by previous arguments this implies $\mathbf{x}=\boldsymbol{\theta}_{i}$, which is a contradiction. From properties of $u_{A t}$ and $u_{B t}$, we know that $-\frac{u_{A t}^{\prime}(x)}{u_{B t}^{\prime}(x)}$ is continuous on $\left(\theta_{A t}, \theta_{B t}\right),-\frac{u_{A t}^{\prime}(x)}{u_{B t}^{\prime}(x)}>0$ for all $x \in\left(\theta_{A t}, \theta_{B t}\right), \lim _{x \rightarrow \theta_{A t}^{+}}-\frac{u_{A t}^{\prime}(x)}{u_{B t}^{\prime}(x)}=0$, $\lim _{x \rightarrow \theta_{B t}^{-}}-\frac{u_{A t}^{\prime}(x)}{u_{B t}^{\prime}(x)}=\infty$ and $\frac{\partial}{\partial x}\left[-\frac{u_{A t}^{\prime}(x)}{u_{B t}^{\prime}(x)}\right]=-\frac{u_{A t}^{\prime \prime}(x) u_{B t}^{\prime}(x)-u_{A}^{\prime}(x) u_{B}^{\prime \prime}(x)}{\left(u_{B t}^{\prime}(x)\right)^{2}}>0$ for all $x \in\left(\theta_{A t}, \theta_{B t}\right)$. Hence, by the Intermediate Value Theorem, a solution to $-\frac{u_{A t}^{\prime}(x)}{u_{B t}^{\prime}(x)}=\lambda^{*}$ exists and by the strict monotonicity of $-\frac{u_{A t}^{\prime}(x)}{u_{B t}^{\prime}(x)}$, it is unique.

\section{A1.4 Proof of Lemma 2}

To show part 1, note that by Proposition 2 part 1, any dynamically Pareto efficient allocation $\mathbf{x}^{*}$ satisfies $x_{1}^{*} \in\left[\theta_{A 1}, \theta_{B 1}\right]$ and $x_{2}^{*} \in\left[\theta_{A 2}, \theta_{B 2}\right]$. If $\theta_{B 1}<\theta_{A 2}$ or $\theta_{B 2}<\theta_{A 1}$, then $x_{1}^{*} \neq x_{2}^{*}$.

Part 2 follows from Lemma A1 below and part 3 follows from Lemma A2 below.

Lemma A1. Suppose utilities have increasing marginal returns. If $\theta_{i t^{\prime}}>\theta_{i t^{\prime \prime}}$ for all $i \in$ $\{A, B\}$, then any dynamically Pareto efficient allocation $\mathbf{x}^{*}$ satisfies $x_{t^{\prime}}^{*}>x_{t^{\prime \prime}}^{*}$.

Proof. Since $\mathbf{x}^{*}$ is dynamically Pareto efficient, it follows that for some $\alpha \in[0,1], \mathbf{x}^{*}$ is a solution to the following maximization problem

$$
\max _{\mathbf{x} \in \mathbb{R}_{+}^{T}} \alpha U_{A}(\mathbf{x})+(1-\alpha) U_{B}(\mathbf{x}),
$$

which implies that $x_{t}^{*}$ is a solution to the following maximization problem

$$
\max _{x_{t} \in \mathbb{R}_{+}} \alpha u_{A}\left(x_{t}, \theta_{A t}\right)+(1-\alpha) u_{B}\left(x_{t}, \theta_{B t}\right)
$$

for all $t$. 
Let $f\left(x_{t}, \theta_{A t}, \theta_{B t}\right)=\alpha u_{A}\left(x_{t}, \theta_{A t}\right)+(1-\alpha) u_{B}\left(x_{t}, \theta_{B t}\right)$. Since $\frac{\partial u_{i}}{\partial x_{t}}$ is strictly increasing in $\theta_{i t}$ for all $i$, we have that $\frac{\partial f}{\partial x_{t}}$ is strictly increasing in $\theta_{i t}$ for all $i$. It follows from standard monotone comparative statics results (for example, Edlin and Shannon, 1998, Theorem 3) that if $\theta_{i t^{\prime}}>\theta_{i t^{\prime \prime}}$ for all $i \in\{A, B\}$, then $x_{t^{\prime}}^{*}>x_{t^{\prime \prime}}^{*}$.

Lemma A2. Suppose $u_{i t}\left(x_{t}\right)=-\left(\left|x_{t}-\theta_{i t}\right|\right)^{r}$ where $r>1$. Then an allocation $\mathbf{x}$ is dynamically Pareto efficient if and only if $\mathbf{x}=\alpha \boldsymbol{\theta}_{i}+(1-\alpha) \boldsymbol{\theta}_{j}$ for some $\alpha \in[0,1]$.

Proof. Fix $i, j \in\{A, B\}$ with $i \neq j$ in (DSP). For any $\bar{U}>U_{j}\left(\boldsymbol{\theta}_{j}\right)$ the solution to (DSP) does not exist, so assume $\bar{U} \leq U_{j}\left(\boldsymbol{\theta}_{j}\right)$.

Suppose $\boldsymbol{\theta}_{i}=\boldsymbol{\theta}_{j}$. Then for any $\bar{U} \leq U_{j}\left(\boldsymbol{\theta}_{j}\right)$ there exists a unique solution to (DSP), $\mathbf{x}^{*}=\boldsymbol{\theta}_{i}$. In this case $\mathbf{x}^{*}=\alpha \boldsymbol{\theta}_{i}+(1-\alpha) \boldsymbol{\theta}_{j}$ for any $\alpha \in[0,1]$.

Suppose $\boldsymbol{\theta}_{i} \neq \boldsymbol{\theta}_{j}$. For $\bar{U}=U_{j}\left(\boldsymbol{\theta}_{j}\right)$, the solution is $\mathbf{x}^{*}=\boldsymbol{\theta}_{j}=\alpha \boldsymbol{\theta}_{i}+(1-\alpha) \boldsymbol{\theta}_{j}$ where $\alpha=0$. For any $\bar{U} \leq U_{j}\left(\boldsymbol{\theta}_{i}\right)$, the solution is $\mathbf{x}^{*}=\boldsymbol{\theta}_{i}=\alpha \boldsymbol{\theta}_{i}+(1-\alpha) \boldsymbol{\theta}_{j}$ where $\alpha=1$.

For the rest of the proof, suppose $\bar{U} \in\left(U_{j}\left(\boldsymbol{\theta}_{i}\right), U_{j}\left(\boldsymbol{\theta}_{j}\right)\right)$ and $\boldsymbol{\theta}_{i} \neq \boldsymbol{\theta}_{j}$. Consider a relaxed version of (DSP) with the constraint $\mathbf{x} \in \mathbb{R}_{+}^{T}$ dropped. Denote the solution to the relaxed problem as $\hat{\mathbf{x}}=\left\{\hat{x}_{t}\right\}_{t=1}^{T}$. Similar to the proof of Proposition 2 part 1 , if $\hat{x}_{t} \notin\left[\theta_{A t}, \theta_{B t}\right]$, then both parties can be made strictly better off in period $t$. Thus if $\hat{\mathbf{x}}$ is a solution to the relaxed problem, then $\hat{x}_{t} \in\left[\theta_{A t}, \theta_{B t}\right]$ for all $t$. It follows that the solution to the relaxed problem is the same as the solution to (DSP). The Lagrangian of the relaxed problem is $L(\mathbf{x}, \lambda)=U_{i}(\mathbf{x})-\lambda\left[-U_{j}(\mathbf{x})+\bar{U}\right]$. Since $U_{j}(\mathbf{x})>\bar{U}$ when $\mathbf{x}=\boldsymbol{\theta}_{j}$, by Takayama (1974, Theorem 1.D.2), $\frac{\partial L(\hat{\mathbf{x}}, \hat{\lambda})}{\partial x_{t}}=0$ with $\hat{\lambda} \geq 0$ for all $t$ is both sufficient and necessary for $\hat{\mathbf{x}}$.

Assume $i=A$. If $i=B$, the argument is similar and omitted. Since $\hat{x}_{t} \in\left[\theta_{A t}, \theta_{B t}\right]$ for all $t$, we have $\frac{\partial L(\hat{\mathbf{x}}, \hat{\lambda})}{\partial x_{t}} \frac{1}{r}=-\left(\hat{x}_{t}-\theta_{A t}\right)^{r-1}+\hat{\lambda}\left(\theta_{B t}-\hat{x}_{t}\right)^{r-1}$. Hence $\frac{\partial L(\hat{\mathbf{x}}, \hat{\lambda})}{\partial x_{t}}=0$ is equivalent to $-\left(\hat{x}_{t}-\theta_{A t}\right)^{r-1}+\hat{\lambda}\left(\theta_{B t}-\hat{x}_{t}\right)^{r-1}=0$. If $\hat{\lambda}=0$, we obtain $\hat{\mathbf{x}}=\boldsymbol{\theta}_{A}$, violating the $U_{B}(\mathbf{x}) \geq \bar{U}$ constraint, and hence $\hat{\lambda}>0$. If $\theta_{A t} \neq \theta_{B t}$, then $\frac{\partial L(\hat{\mathbf{x}}, \hat{\lambda})}{\partial x_{t}}=0$ cannot be satisfied if $\hat{x}_{t}=\theta_{A t}$ or $\hat{x}_{t}=\theta_{B t}$. Hence for all $t$ such that $\theta_{A t} \neq \theta_{B t}$, we have $\hat{x}_{t} \in\left(\theta_{A t}, \theta_{B t}\right)$ and therefore $\frac{\hat{x}_{t}-\theta_{A t}}{\theta_{B t}-\hat{x}_{t}}=\hat{\lambda}^{\frac{1}{r-1}}$ with $\hat{\lambda}>0$.

Fix $t^{\prime}$ such that $\theta_{B t^{\prime}} \neq \theta_{A t^{\prime}}$. If $\theta_{A t} \neq \theta_{B t}$, then $\theta_{B t}-\hat{x}_{t}=\left(\theta_{B t^{\prime}}-\hat{x}_{t^{\prime}}\right) \frac{\theta_{B t}-\theta_{A t}}{\theta_{B t^{\prime}}-\theta_{A t^{\prime}}}$ since $\frac{\hat{x}_{t}-\theta_{A t}}{\theta_{B t}-\hat{x}_{t}}=$ $\hat{\lambda}^{\frac{1}{r-1}}$ and $\frac{\hat{x}_{t^{\prime}}-\theta_{A t^{\prime}}}{\theta_{B t^{\prime}}-\hat{x}_{t^{\prime}}}=\hat{\lambda}^{\frac{1}{r-1}}$. If $\theta_{A t}=\theta_{B t}$, then $\hat{x}_{t}=\theta_{B t}$, and so again we have $\theta_{B t}-\hat{x}_{t}=\left(\theta_{B t^{\prime}}-\right.$ 
$\left.\hat{x}_{t^{\prime}}\right) \frac{\theta_{B t}-\theta_{A t}}{\theta_{B t^{\prime}}-\theta_{A t^{\prime}}}$. Thus $U_{B}(\hat{\mathbf{x}})=\left(\frac{\theta_{B t^{\prime}}-\hat{x}_{t^{\prime}}}{\theta_{B t^{\prime}}-\theta_{A t^{\prime}}}\right)^{r} \sum_{t=1}^{T} \delta^{t-1}\left(-\left(\theta_{B t}-\theta_{A t}\right)^{r}\right)=\left(\frac{\theta_{B t^{\prime}}-\hat{x}_{t^{\prime}}}{\theta_{B t^{\prime}}-\theta_{A t^{\prime}}}\right)^{r} U_{B}\left(\boldsymbol{\theta}_{A}\right)$. Since $\hat{\lambda}>0$, we have $U_{B}(\hat{\mathbf{x}})=\bar{U}$. Hence, $\left(\frac{\theta_{B t^{\prime}}-\hat{x}_{t^{\prime}}}{\theta_{B t^{\prime}}-\theta_{A t^{\prime}}}\right)^{r} U_{B}\left(\boldsymbol{\theta}_{A}\right)=\bar{U}$ is necessary and sufficient for $\hat{\mathbf{x}}$ to solve (DSP) with $\bar{U}$. Rearranging, we get $\hat{x}_{t^{\prime}}=\alpha \theta_{A t^{\prime}}+(1-\alpha) \theta_{B t^{\prime}}$ where $\alpha=$ $\left(\frac{\bar{U}}{U_{B}\left(\boldsymbol{\theta}_{A}\right)}\right)^{\frac{1}{r}}$. To conclude the proof, note that if $U_{B}\left(\boldsymbol{\theta}_{A}\right)<\bar{U}<U_{B}\left(\boldsymbol{\theta}_{B}\right)=0$, then $\alpha \in(0,1)$, and conversely, for any $\alpha \in(0,1)$, the allocation $\mathbf{x}=\alpha \boldsymbol{\theta}_{A}+(1-\alpha) \boldsymbol{\theta}_{B}$ solves (DSP) with $\bar{U}=\alpha^{r} U_{B}\left(\boldsymbol{\theta}_{A}\right)$.

\section{A2 Discretionary spending}

The following lemma is useful in the proof of Proposition 3.

Lemma A3. Suppose there exists at most one $t^{\prime}$ such that $\theta_{A t^{\prime}} \neq \theta_{B t^{\prime}}$. Then an allocation $\mathbf{x}$ is dynamically Pareto efficient if and only if $x_{t}$ is statically Pareto efficient in period $t$ for all $t$.

Proof. The "only if" part follows from Proposition 2 part 1 which states that if $\mathbf{x}=\left\{x_{t}\right\}_{t=1}^{T}$ is dynamically Pareto efficient, then $x_{t}$ satisfies static Pareto efficiency for all $t$.

To show the "if" part, suppose $\mathbf{x}=\left\{x_{t}\right\}_{t=1}^{T}$ is an allocation such that $x_{t}$ is statically Pareto efficient in period $t$ for all $t$. The proof is trivial if $\boldsymbol{\theta}_{A}=\boldsymbol{\theta}_{B}$, so we consider the case when there is a unique $t^{\prime}$ such that $\theta_{A t^{\prime}} \neq \theta_{B t^{\prime}}$. We will show that $\mathbf{x}$ solves (DSP). Since $\theta_{A t}=\theta_{B t}$ for $t \neq t^{\prime}$, by Proposition $1, x_{t}=\theta_{A t}$ for all $t \neq t^{\prime}$ and $x_{t^{\prime}}$ solves

$$
\begin{aligned}
\max _{x \in \mathbb{R}_{+}} & u_{i t^{\prime}}(x) \\
\text { s.t. } & u_{j t^{\prime}}(x) \geq \bar{u}
\end{aligned}
$$

for some $\bar{u}$. Since $x_{t^{\prime}}$ solves the problem above, it also solves

$$
\begin{aligned}
\max _{x \in \mathbb{R}_{+}} & \delta^{t^{\prime}-1} u_{i t^{\prime}}(x)+\sum_{t \neq t^{\prime}} \delta^{t-1} u_{i t}\left(\theta_{A t}\right) \\
\text { s.t. } & \delta^{t^{\prime}-1} u_{j t^{\prime}}(x)+\sum_{t \neq 1} \delta^{t-1} u_{j t}\left(\theta_{A t}\right) \geq \delta^{t^{\prime}-1} \bar{u}+\sum_{t \neq t^{\prime}} \delta^{t-1} u_{j t}\left(\theta_{A t}\right) .
\end{aligned}
$$

Since $\left\{\theta_{A t}\right\}_{t \neq t^{\prime}}$ maximizes $\sum_{t \neq t^{\prime}} \delta^{t-1} u_{i t}\left(x_{t}\right)$ and $\sum_{t \neq t^{\prime}} \delta^{t-1} u_{j t}\left(x_{t}\right)$, it follows that $\mathbf{x}$ solves (DSP) with $\bar{U}=\delta^{t^{\prime}-1} \bar{u}+\sum_{t \neq t^{\prime}} \delta^{t-1} u_{j t}\left(\theta_{A t}\right)$. 


\section{A2.1 Proof of Proposition 3}

We first characterize the equilibrium. Since the status quo spending is zero, party $A$ accepts any proposal $k_{t}$ such that $0 \leq k_{t} \leq \phi_{A t}^{o}$. To find party $B$ 's optimal proposal, there are two cases to consider. (i) Suppose $\theta_{B t} \leq \phi_{A t}^{o}$. Then, since $u_{A t}$ is single-peaked at $\theta_{A t} \leq \theta_{B t}$, we have $u_{A t}\left(\theta_{B t}\right) \geq u_{A t}\left(\phi_{A t}^{o}\right)=u_{A t}(0)$ and therefore party $A$ accepts $k_{t}=\theta_{B t}$ in period $t$. In this case, party $B$ 's optimal proposal in period $t$ is equal to its ideal point $\theta_{B t}$. (ii) Suppose $\theta_{B t}>\phi_{A t}^{o}$. Then, given the single-peakedness of $u_{B t}$, the optimal policy for $B$ that is acceptable to $A$ is equal to $\phi_{A t}^{o}$. In this case, party $B$ proposes $k_{t}=\phi_{A t}^{o}<\theta_{B t}$. Hence, party $B$ 's optimal proposal is $\min \left\{\theta_{B t}, \phi_{A t}^{o}\right\}$.

Note that the policy implemented in period $t$ is equal to $\theta_{A t}$ when party $A$ is the proposer and is equal to $\min \left\{\theta_{B t}, \phi_{A t}^{o}\right\} \geq \theta_{A t}$ when party $B$ is the proposer. It follows that the policy implemented in period $t$ is in $\left[\theta_{A t}, \theta_{B t}\right]$ and therefore is statically Pareto efficient by Proposition 1.

For the equilibrium's dynamic efficiency properties, consider the following two cases. (i) Suppose $\theta_{A t} \neq \theta_{B t}$ for all $t$. In this case, $\min \left\{\theta_{B t}, \phi_{A t}^{o}\right\}>\theta_{A t}$, which implies that the policy implemented in period $t$ varies with the identity of the proposer. By Lemma 1, this implies dynamic Pareto inefficiency. (ii) Suppose there is at most one $t^{\prime}$ such that $\theta_{A t^{\prime}} \neq \theta_{B t^{\prime}}$. In this case, by Lemma A3, an allocation $\mathbf{x}$ is dynamically Pareto efficient if and only if $x_{t}$ is statically Pareto efficient in all periods. Thus, the equilibrium is dynamically Pareto efficient.

\section{A3 Mandatory spending}

\section{A3.1 Proof of Proposition 4}

We first prove equilibrium existence by showing that a solution exists for the proposer's problem in period 2 given any status quo $g_{1}$, and given this solution, a solution exists for the proposer's problem in period 1.

Consider the problem of the proposing party $i \in\{A, B\}$ in the second period under status quo $g_{1} \in \mathbb{R}_{+}$and budgetary institution $Z$ that allows for mandatory spending. The proposing 
party's maximization problem is:

$$
\begin{aligned}
\max _{\left(k_{2}, g_{2}\right) \in Z} & u_{i 2}\left(k_{2}+g_{2}\right) \\
\text { s.t. } & u_{j 2}\left(k_{2}+g_{2}\right) \geq u_{j 2}\left(g_{1}\right) .
\end{aligned}
$$

Consider the related problem

$$
\max _{x_{2} \in A_{j 2}\left(g_{1}\right)} u_{i 2}\left(x_{2}\right)
$$

where $A_{j 2}\left(g_{1}\right)=\left\{x \in \mathbb{R}_{+} \mid u_{j 2}(x) \geq u_{j 2}\left(g_{1}\right)\right\}$ is the responder's acceptance set under status quo $g_{1}$. If $\hat{x}_{2}$ is a solution to $\left(P_{2}^{\prime}\right)$, then any $\left(\hat{k}_{2}, \hat{g}_{2}\right) \in Z$ such that $\hat{k}_{2}+\hat{g}_{2}=\hat{x}_{2}$ is a solution to $\left(P_{2}\right)$. We use the following properties of $A_{j 2}$.

Lemma A4. $A_{j 2}\left(g_{1}\right)$ is non-empty, convex and compact for any $g_{1} \in \mathbb{R}_{+}$and $A_{j 2}$ is continuous.

Proof. Non-emptiness follows from $g_{1} \in A_{j 2}\left(g_{1}\right)$ for all $g_{1} \in \mathbb{R}_{+}$. Convexity follows from strict concavity of $u_{j 2}$. To show compactness, we show that $A_{j 2}\left(g_{1}\right)$ is closed and bounded for all $g_{1} \in \mathbb{R}_{+}$. Closedness follows from continuity of $u_{j 2}$. For boundedness, note that $u_{j 2}$ is differentiable and strictly concave, which implies that $u_{j 2}(x)<u_{j 2}(y)+u_{j 2}^{\prime}(y)(x-y)$ for any $x, y \in \mathbb{R}_{+}$. Selecting $y>\theta_{j 2}$ gives $u_{j 2}^{\prime}(y)<0$ and taking the limit as $x \rightarrow \infty$, we have $\lim _{x \rightarrow \infty} u_{j 2}(x)=-\infty$. We next establish upper and lower hemicontinuity of $A_{j 2}$ using Lemma A5.

Lemma A5. Let $X \subseteq \mathbb{R}$ be closed and convex, let $Y \subseteq \mathbb{R}$ and let $f: X \rightarrow Y$ be a continuous function. Define $\varphi: X \rightarrow X$ by

$$
\varphi(x)=\{y \in X \mid f(y) \geq f(x)\}
$$

1. If $\varphi(x)$ is compact $\forall x \in X$, then $\varphi$ is upper hemicontinuous.

2. If $f$ is strictly concave, then $\varphi$ is lower hemicontinuous.

Proof. To show part 1 , since $\varphi(x)$ is compact for all $x \in X$, it suffices to prove that if $x_{n} \rightarrow x$ and $y_{n} \rightarrow y$ with $y_{n} \in \varphi\left(x_{n}\right)$ for all $n \in \mathbb{N}$, then $y \in \varphi(x)$. Since $y_{n} \in \varphi\left(x_{n}\right)$, we have $f\left(y_{n}\right) \geq f\left(x_{n}\right)$. Since $f$ is continuous, $x_{n} \rightarrow x$ and $y_{n} \rightarrow y$, it follows that $f(y) \geq f(x)$, hence $y \in \varphi(x)$. 
To show part 2, fix $x \in X$, let $y \in \varphi(x)$ and consider any $x_{n} \rightarrow x$. We show that there exists a sequence $y_{n} \rightarrow y$ and $n^{\prime}$ such that $y_{n} \in \varphi\left(x_{n}\right)$ for all $n \geq n^{\prime}$. First suppose $f(y)>f(x)$. Set $y_{n}=y$. Clearly, $y_{n} \rightarrow y$. By continuity of $f$, there exists $n^{\prime}$ such that $f\left(y_{n}\right) \geq f\left(x_{n}\right)$ for all $n \geq n^{\prime}$, that is, $y_{n} \in \varphi\left(x_{n}\right)$. Next suppose $f(y)=f(x)$. There are two cases to consider. First, if $y=x$, set $y_{n}=x_{n}$. Clearly $y_{n} \rightarrow y$ and $y_{n} \in \varphi\left(x_{n}\right)$ for all $n$. Second, suppose $y \neq x$. By strict concavity of $f$, there exist at most one such $y \in X$. Set $y_{n}=y$ whenever $f\left(x_{n}\right) \leq f(x)$. When $f\left(x_{n}\right)>f(x)$, by strict concavity of $f$ and the Intermediate Value Theorem, because $x_{n} \rightarrow x$, there exists $n^{\prime}$ such that for all $n \geq n^{\prime}$, there is a unique $y_{n} \neq x_{n}$ such that $f\left(y_{n}\right)=f\left(x_{n}\right)>f(x)$. Because $y_{n}=y$ whenever $f\left(x_{n}\right) \leq f(x)=f(y)$ and $f\left(y_{n}\right)=f\left(x_{n}\right)$ whenever $f\left(x_{n}\right)>f(x), y_{n} \in \varphi\left(x_{n}\right)$ for all $n \geq n^{\prime}$ and $y_{n} \rightarrow y$ follows from continuity of $f$.

To see that $A_{j 2}$ is upper and lower hemicontinuous, note that it can be written as $\varphi$ in (A4) with $X=\mathbb{R}_{+}$closed and convex, $Y=\mathbb{R}$ and $f=u$ continuous and strictly concave, and we showed before that $A_{j 2}$ is compact-valued.

By Lemma A4, for any $g_{1} \in \mathbb{R}_{+}$, the acceptance set $A_{j 2}\left(g_{1}\right)$ is non-empty and compact. Applying the Weierstrass's Theorem, a solution exists for $\left(P_{2}^{\prime}\right)$.

We next show that a solution exists to the proposer's problem in period 1. Recall the continuation value $V_{i}$ is given by

$$
V_{i}\left(g_{1} ; \sigma_{2}^{*}\right)=p_{A} u_{i 2}\left(\kappa_{A 2}^{*}\left(g_{1}\right)+\gamma_{A 2}^{*}\left(g_{1}\right)\right)+p_{B} u_{i 2}\left(\kappa_{B 2}^{*}\left(g_{1}\right)+\gamma_{B 2}^{*}\left(g_{1}\right)\right),
$$

where $\kappa_{i 2}^{*}\left(g_{1}\right)+\gamma_{i 2}^{*}\left(g_{1}\right)$ is a solution to $\left(P_{2}^{\prime}\right)$ for all $i \in\{A, B\}$. For any $g_{1} \in \mathbb{R}_{+}$, and $i \in\{A, B\}$, let $V_{i}\left(g_{1}\right)=V_{i}\left(g_{1} ; \sigma_{2}^{*}\right)$ and

$$
\begin{gathered}
F_{i}\left(k_{1}, g_{1}\right)=u_{i 1}\left(k_{1}+g_{1}\right)+\delta V_{i}\left(g_{1}\right), \\
f_{i}\left(g_{1}\right)=u_{i 1}\left(g_{1}\right)+\delta V_{i}\left(g_{1}\right) .
\end{gathered}
$$

Lemma A6 establishes some properties of $V_{i}, F_{i}$ and $f_{i}$.

Lemma A6. $V_{i}, F_{i}$ and $f_{i}$ are continuous. $V_{i}$ is bounded.

Proof. To show continuity of $V_{i}$, first note that given $u_{i 2}$ is strictly concave, the solution to $\left(P_{2}^{\prime}\right)$ is unique for any $g_{1} \in \mathbb{R}_{+}$. Since $A_{j 2}$ is non-empty, compact valued and is contin- 
uous by Lemma A4, applying the Maximum Theorem we have that the correspondence of maximizers in $\left(P_{2}^{\prime}\right)$ is upper hemicontinuous. Since a singleton-valued upper hemicontinuous correspondence is continuous as a function, $\kappa_{i 2}^{*}+\gamma_{i 2}^{*}$ is continuous. Thus $V_{i}\left(g_{1}\right)$ is continuous. Continuity of $F_{i}$ and $f_{i}$ follow from their definitions and continuity of $V_{i}$.

To show boundedness of $V_{i}$, first note that $u_{i 2}\left(\kappa_{k 2}^{*}\left(g_{1}\right)+\gamma_{k 2}^{*}\left(g_{1}\right)\right) \leq u_{i 2}\left(\theta_{i 2}\right)$ for all $k \in$ $\{A, B\}$ and $g_{1} \in \mathbb{R}_{+}$because $\theta_{i 2}$ is the unique maximizer of $u_{i 2}$. Moreover, for $i \neq j$, if $u_{i 2}\left(\kappa_{k 2}^{*}\left(g_{1}\right)+\gamma_{k 2}^{*}\left(g_{1}\right)\right)<u_{i 2}\left(\theta_{j 2}\right)$ for some $k \in\{A, B\}$ and some $g_{1} \in \mathbb{R}_{+}$, then $k$ could make an alternative proposal that the responder would accept and $k$ would strictly prefer. It follows that $u_{i 2}\left(\kappa_{k 2}^{*}\left(g_{1}\right)+\gamma_{k 2}^{*}\left(g_{1}\right)\right) \geq u_{i 2}\left(\theta_{j 2}\right)$. Thus $V_{i}\left(g_{1}\right) \in\left[u_{i 2}\left(\theta_{j 2}\right), u_{i 2}\left(\theta_{i 2}\right)\right]$ for any $g_{1}$.

Fix the initial status quo $g_{0} \in \mathbb{R}_{+}$. When only mandatory spending programs are allowed, $Z=\{0\} \times \mathbb{R}_{+}$, and party $i$ 's equilibrium proposal satisfies $\kappa_{i 1}^{*}\left(g_{0}\right)=0$ and

$$
\gamma_{i 1}^{*}\left(g_{0}\right) \in \underset{g_{1} \in \mathbb{R}_{+}}{\arg \max } f_{i}\left(g_{1}\right) \text { s.t. } f_{j}\left(g_{1}\right) \geq f_{j}\left(g_{0}\right) .
$$

When both types of spending are allowed, we have $Z=\mathbb{R}_{+} \times \mathbb{R}_{+}$or $Z=\left\{\left(k_{t}, g_{t}\right) \in \mathbb{R} \times\right.$ $\left.\mathbb{R}_{+} \mid k_{t}+g_{t} \geq 0\right\}$, depending on whether discretionary spending can be negative. In equilibrium party $i$ 's proposal satisfies

$$
\left(\kappa_{i 1}^{*}\left(g_{0}\right), \gamma_{i 1}^{*}\left(g_{0}\right)\right) \in \underset{\left(k_{1}, g_{1}\right) \in Z}{\arg \max } F_{i}\left(k_{1}, g_{1}\right) \quad \text { s.t. } \quad F_{j}\left(k_{1}, g_{1}\right) \geq F_{j}\left(0, g_{0}\right) .
$$

We show that in each of these problems, the objective function is continuous and the constraint set is compact for any $g_{0} \in \mathbb{R}_{+}$. Lemma A6 establishes continuity of $F_{i}$ and $f_{i}$ and boundedness of $V_{i}$. Compactness follows from an argument analogous to the one made for the second period. Hence for any $g_{0} \in \mathbb{R}_{+}$, a solution to each of the optimization problems exists, and therefore an equilibrium exists.

We now show that for any $g_{1} \in \mathbb{R}_{+}$

$$
\begin{aligned}
\kappa_{A 2}^{*}\left(g_{1}\right)+\gamma_{A 2}^{*}\left(g_{1}\right) & =\max \left\{\theta_{A 2}, \phi_{B 2}\left(g_{1}\right)\right\} \\
\kappa_{B 2}^{*}\left(g_{1}\right)+\gamma_{B 2}^{*}\left(g_{1}\right) & =\min \left\{\theta_{B 2}, \phi_{A 2}\left(g_{1}\right)\right\}
\end{aligned}
$$

Consider (A9). The proof of (A10) is similar and omitted. Note that $\phi_{B 2}\left(g_{1}\right)=\min \{x \in$ $\left.\mathbb{R}_{+} \mid u_{B 2}(x) \geq u_{B 2}\left(g_{1}\right)\right\}=\min A_{B 2}\left(g_{1}\right)$. There are two possible cases. 
Case (i): $\phi_{B 2}\left(g_{1}\right) \leq \theta_{A 2}$. Since $\theta_{B 2} \in A_{B 2}\left(g_{1}\right), \phi_{B 2}\left(g_{1}\right) \in A_{B 2}\left(g_{1}\right)$ and $\theta_{A 2} \in\left[\phi_{B 2}\left(g_{1}\right), \theta_{B 2}\right]$, by convexity of $A_{B 2}\left(g_{1}\right)$, we have $\theta_{A 2} \in A_{B 2}\left(g_{1}\right)$. Since $\theta_{A 2}$ maximizes $u_{A 2}$, it follows that $\hat{x}_{2}=\theta_{A 2}=\max \left\{\theta_{A 2}, \phi_{B 2}\left(g_{1}\right)\right\}$.

Case (ii): $\phi_{B 2}\left(g_{1}\right)>\theta_{A 2}$. Since $\phi_{B 2}\left(g_{1}\right)=\min A_{B 2}\left(g_{1}\right)$, for any $x<\phi_{B 2}\left(g_{1}\right)$ we have $x \notin A_{B 2}\left(g_{1}\right)$. Since $u_{A 2}(x)$ is strictly decreasing for $x>\theta_{A 2}$, we have $\hat{x}_{2}=\phi_{B 2}\left(g_{1}\right)=$ $\max \left\{\theta_{A 2}, \phi_{B 2}\left(g_{1}\right)\right\}$.

We now prove parts 1 and 2. For part 1, let $Z$ be a budgetary institution that allows mandatory spending. If $\kappa_{i 2}^{*}\left(g_{1}\right)+\gamma_{i 2}^{*}\left(g_{1}\right) \notin\left[\theta_{A 2}, \theta_{B 2}\right]$, there exists $\left(k_{2}^{\prime}, g_{2}^{\prime}\right) \in Z$ with $k_{2}^{\prime}+g_{2}^{\prime} \in$ $\left[\theta_{A 2}, \theta_{B 2}\right]$ such that $u_{A 2}\left(\kappa_{i 2}^{*}\left(g_{1}\right)+\gamma_{i 2}^{*}\left(g_{1}\right)\right)<u_{A 2}\left(k_{2}^{\prime}+g_{2}^{\prime}\right)$ and $u_{B 2}\left(\kappa_{i 2}^{*}\left(g_{1}\right)+\gamma_{i 2}^{*}\left(g_{1}\right)\right)<u_{B 2}\left(k_{2}^{\prime}+\right.$ $\left.g_{2}^{\prime}\right)$. Hence $\kappa_{i 2}^{*}\left(g_{1}\right)+\gamma_{i 2}^{*}\left(g_{1}\right) \in\left[\theta_{A 2}, \theta_{B 2}\right]$.

For part 2, assume $\theta_{A 2} \neq \theta_{B 2}$. There are three possible cases.

Case (i): $g_{1} \in\left[\theta_{A 2}, \theta_{B 2}\right]$. Because $u_{A 2}\left(k_{2}+g_{2}\right)<u_{A 2}\left(g_{1}\right)$ for any $k_{2}+g_{2}>g_{1}$ and $u_{B 2}\left(k_{2}+g_{2}\right)<u_{B 2}\left(g_{1}\right)$ for any $k_{2}+g_{2}<g_{1}$, party $A$ does not propose or accept any proposal such that $k_{2}+g_{2}>g_{1}$ and party $B$ does not propose or accept any proposal such that $k_{2}+g_{2}<g_{1}$. Hence $\kappa_{A 2}^{*}\left(g_{1}\right)+\gamma_{A 2}^{*}\left(g_{1}\right)=\kappa_{B 2}^{*}\left(g_{1}\right)+\gamma_{B 2}^{*}\left(g_{1}\right)=g_{1}$.

Case (ii): $g_{1}<\theta_{A 2}$. In this case $\kappa_{A 2}^{*}\left(g_{1}\right)+\gamma_{A 2}^{*}\left(g_{1}\right)=\theta_{A 2}$ since $\theta_{A 2}$ is the unique maximizer of $u_{A 2}$ and $u_{B 2}\left(\theta_{A 2}\right)>u_{B 2}\left(g_{1}\right)$. By the definition of $\phi_{A 2}$ we have $\phi_{A 2}\left(g_{1}\right)>\theta_{A 2}$ when $g_{1}<\theta_{A 2}$. Hence from (A10) we have $\kappa_{B 2}^{*}\left(g_{1}\right)+\gamma_{B 2}^{*}\left(g_{1}\right)>\theta_{A 2}$.

Case (iii): $g_{1}>\theta_{B 2}$. Using an argument analogous to case (ii), we have $\kappa_{B 2}^{*}\left(g_{1}\right)+$ $\gamma_{B 2}^{*}\left(g_{1}\right)=\theta_{B 2}$ and $\kappa_{A 2}^{*}\left(g_{1}\right)+\gamma_{A 2}^{*}\left(g_{1}\right)<\theta_{B 2}$.

\section{A3.2 Proof of Proposition 5}

Note that the proposition applies only when $\theta_{A t} \neq \theta_{B t}$ for all $t$. We first prove parts 1 and 2. Assume, towards a contradiction, that $\sigma^{*}$ is a dynamically Pareto efficient equilibrium given $g_{0} \in \mathbb{R}_{+}$. The following lemma is useful.

Lemma A7. Let $Z=\{0\} \times \mathbb{R}_{+}$. Suppose $\theta_{A t} \neq \theta_{B t}$ for all $t$. For any $g_{0} \in \mathbb{R}_{+}$, if $\sigma^{*}$ is a dynamically Pareto efficient equilibrium given $g_{0}$, then any equilibrium allocation $\mathbf{x}^{\sigma^{*}}\left(g_{0}\right)$ satisfies $x_{1}^{\sigma^{*}}\left(g_{0}\right)=x_{2}^{\sigma^{*}}\left(g_{0}\right)$.

Proof. Fix a dynamically Pareto efficient equilibrium $\sigma^{*}$ given $g_{0}$. The equilibrium spending 
in period 2 is either $\gamma_{A 2}^{*}\left(x_{1}^{\sigma^{*}}\left(g_{0}\right)\right)$ or $\gamma_{B 2}^{*}\left(x_{1}^{\sigma^{*}}\left(g_{0}\right)\right)$. Since $\sigma^{*}$ is an equilibrium and $\theta_{A t} \neq \theta_{B t}$ for all $t$, we have either $\gamma_{A 2}^{*}\left(x_{1}^{\sigma^{*}}\left(g_{0}\right)\right) \neq \gamma_{B 2}^{*}\left(x_{1}^{\sigma^{*}}\left(g_{0}\right)\right)$ or $x_{2}^{\sigma^{*}}\left(g_{0}\right)=\gamma_{A 2}^{*}\left(x_{1}^{\sigma^{*}}\left(g_{0}\right)\right)=\gamma_{B 2}^{*}\left(x_{1}^{\sigma^{*}}\left(g_{0}\right)\right)=$ $x_{1}^{\sigma^{*}}\left(g_{0}\right)$ by Proposition 4 part 2 . In the former case, the level of spending in period 2 depends on the identity of the period-2 proposer, contradicting that $\sigma^{*}$ is a dynamically Pareto efficient equilibrium given $g_{0}$ by Lemma 1 . Thus, we must have $x_{1}^{\sigma^{*}}\left(g_{0}\right)=x_{2}^{\sigma^{*}}\left(g_{0}\right)$.

By Lemma A7, we have $x_{1}^{\sigma^{*}}\left(g_{0}\right)=x_{2}^{\sigma^{*}}\left(g_{0}\right)$. To see part 1 , note that Proposition 2 part 1 implies that $x_{1}^{\sigma^{*}}\left(g_{0}\right) \in\left[\theta_{A 1}, \theta_{B 1}\right]$ and $x_{2}^{\sigma^{*}}\left(g_{0}\right) \in\left[\theta_{A 2}, \theta_{B 2}\right]$, which is impossible if $\theta_{A 1}<\theta_{B 1}<$ $\theta_{A 2}<\theta_{B 2}$ or if $\theta_{A 2}<\theta_{B 2}<\theta_{A 1}<\theta_{B 1}$. To see part $2,\left(x_{1}^{\sigma^{*}}\left(g_{0}\right), x_{2}^{\sigma^{*}}\left(g_{0}\right)\right)$ is not a dynamically Pareto efficient allocation since any dynamically Pareto efficient allocation satisfies $x_{1}^{*} \neq x_{2}^{*}$ when parties' ideals are increasing or decreasing and $\theta_{A t} \neq \theta_{B t}$ for all $t$ by Lemma A1.

We next prove the remaining result. We first show that for any equilibrium $\sigma^{*}$, there exists $g_{0}$ such that $\sigma^{*}$ is dynamically Pareto efficient given $g_{0}$. To show that, we make use of the following lemma.

Lemma A8. Let $Z=\{0\} \times \mathbb{R}_{+}$. Suppose $(\tilde{x}, \tilde{x})$ is a dynamically Pareto efficient allocation. If $g_{t-1}=\tilde{x}$, then $\gamma_{i t}^{*}\left(g_{t-1}\right)=\tilde{x}$ for all $i \in\{A, B\}$ and all $t$ in any equilibrium.

Proof. Consider the second period. Since $(\tilde{x}, \tilde{x})$ is dynamically Pareto efficient, $\tilde{x} \in\left[\theta_{A 2}, \theta_{B 2}\right]$ by Proposition 2 part 1 . Thus $\gamma_{i 2}^{*}(\tilde{x})=\tilde{x}$ for all $i \in\{A, B\}$ by Proposition 4 .

Consider the first period. Suppose, towards a contradiction, $\gamma_{i 1}^{*}(\tilde{x})=\hat{x} \neq \tilde{x}$ for some $i \in\{A, B\}$. Note that party $i$ can achieve a dynamic payoff of at least $u_{i 1}(\tilde{x})+\delta u_{i 2}(\tilde{x})$ for all $i \in\{A, B\}$ by following the strategy of always proposing $\tilde{x}$ when proposing and rejecting any proposal when responding. Hence, $u_{j 1}(\hat{x})+\delta V_{j}(\hat{x}) \geq u_{j 1}(\tilde{x})+\delta u_{j 2}(\tilde{x})$ for all $j \in\{A, B\}$ since $\hat{x}$ is an equilibrium proposal.

Note that $V_{j}(\hat{x})=p_{A} u_{j 2}\left(\gamma_{A 2}^{*}(\hat{x})\right)+p_{B} u_{j 2}\left(\gamma_{B 2}^{*}(\hat{x})\right) \leq u_{j 2}\left(\hat{x}^{\prime}\right)$ by strict concavity of $u_{j 2}$ where $\hat{x}^{\prime}=p_{A} \gamma_{A 2}^{*}(\hat{x})+p_{B} \gamma_{B 2}^{*}(\hat{x})$. Hence $u_{j 1}(\hat{x})+\delta u_{j 2}\left(\hat{x}^{\prime}\right) \geq u_{j 1}(\tilde{x})+\delta u_{j 2}(\tilde{x})$ for all $j \in\{A, B\}$. Since the solution to (DSP) is unique for any given $\bar{U}$ (see footnote 18), this contradicts the dynamic efficiency of $(\tilde{x}, \tilde{x})$.

By Lemma A2, $\mathbf{x}$ is a dynamically Pareto efficient allocation if and only if $\mathbf{x}=\alpha \boldsymbol{\theta}_{A}+$ $(1-\alpha) \boldsymbol{\theta}_{B}$ for some $\alpha \in[0,1]$. This implies any dynamically Pareto efficient allocation 
$\left(x_{1}^{*}, x_{2}^{*}\right)$ can be written as $x_{1}^{*}(\alpha)=\alpha \theta_{A 1}+(1-\alpha) \theta_{B 1}$ and $x_{2}^{*}(\alpha)=\alpha \theta_{A 2}+(1-\alpha) \theta_{B 2}$. Let $\alpha^{*}=\frac{\theta_{B 1}-\theta_{B 2}}{\theta_{B 1}-\theta_{B 2}+\theta_{A 2}-\theta_{A 1}}$. Note that $\alpha^{*} \in(0,1) \operatorname{since} \operatorname{sgn}\left[\theta_{B 1}-\theta_{B 2}\right]=\operatorname{sgn}\left[\theta_{A 2}-\theta_{A 1}\right]$ and $\theta_{i 1} \neq \theta_{i 2}$ for all $i \in\{A, B\}$. Note also that $x_{1}^{*}(\alpha)=x_{2}^{*}(\alpha)$ if and only if $\alpha=\alpha^{*}$. By Lemma A8, any equilibrium $\sigma^{*}$ is dynamically Pareto efficient given $g_{0}$ if $g_{0}=x_{1}^{*}\left(\alpha^{*}\right)$.

In the remainder of the proof, we let $\sigma^{*}$ be a dynamically Pareto efficient equilibrium given $g_{0}$ and show that we must have $g_{0}=x_{1}^{*}\left(\alpha^{*}\right)$. We do this in three steps.

Step 1: We show that $\gamma_{A 1}^{*}\left(g_{0}\right) \in\left(\theta_{A 1}, \theta_{B 1}\right) \cap\left(\theta_{A 2}, \theta_{B 2}\right)$. By Lemma A7, we have $x_{1}^{\sigma^{*}}\left(g_{0}\right)=$ $x_{2}^{\sigma^{*}}\left(g_{0}\right)$. Recall that $x_{1}^{*}(\alpha)=x_{2}^{*}(\alpha)$ if and only if $\alpha=\alpha^{*}$. Thus $x_{1}^{\sigma^{*}}\left(g_{0}\right)=x_{2}^{\sigma^{*}}\left(g_{0}\right)=$ $x_{1}^{*}\left(\alpha^{*}\right)=x_{2}^{*}\left(\alpha^{*}\right)$. Note that $x_{1}^{*}\left(\alpha^{*}\right) \in\left(\theta_{A 1}, \theta_{B 1}\right)$ and $x_{2}^{*}\left(\alpha^{*}\right) \in\left(\theta_{A 2}, \theta_{B 2}\right)$ since $\alpha^{*} \in(0,1)$. Since $x_{1}^{\sigma^{*}}\left(g_{0}\right)=x_{1}^{*}\left(\alpha^{*}\right)=x_{2}^{*}\left(\alpha^{*}\right)$ and $\alpha^{*}$ is unique, it follows that $\gamma_{A 1}^{*}\left(g_{0}\right)=x_{1}^{\sigma^{*}}\left(g_{0}\right) \in$ $\left(\theta_{A 1}, \theta_{B 1}\right) \cap\left(\theta_{A 2}, \theta_{B 2}\right)$.

Step 2: We now show that $f_{A}\left(\gamma_{A 1}^{*}\left(g_{0}\right)\right)=f_{A}\left(g_{0}\right)$ and $f_{B}\left(\gamma_{A 1}^{*}\left(g_{0}\right)\right)=f_{B}\left(g_{0}\right)$. To see this, note that $f_{A}\left(\gamma_{A 1}^{*}\left(g_{0}\right)\right) \geq f_{A}\left(g_{0}\right)$ and $f_{B}\left(\gamma_{A 1}^{*}\left(g_{0}\right)\right) \geq f_{B}\left(g_{0}\right)$ since $\gamma_{A 1}^{*}\left(g_{0}\right)$ is proposed by $A$ and accepted by $B$ under status quo $g_{0}$. Suppose, towards a contradiction, that $f_{A}\left(\gamma_{A 1}^{*}\left(g_{0}\right)\right) \geq$ $f_{A}\left(g_{0}\right)$ and $f_{B}\left(\gamma_{A 1}^{*}\left(g_{0}\right)\right)>f_{B}\left(g_{0}\right)$. Since $f_{i}\left(g_{1}\right)=u_{i 1}\left(g_{1}\right)+\delta V_{i}\left(g_{1}\right)$, where $V_{i}\left(g_{1}\right)=u_{i 2}\left(g_{1}\right)$ for all $g_{1} \in\left[\theta_{A 2}, \theta_{B 2}\right]$ by Proposition 4 part $2, f_{A}\left(g_{1}\right)$ is strictly decreasing and $f_{B}\left(g_{1}\right)$ is strictly increasing in $g_{1}$ on $\left[\theta_{A 1}, \theta_{B 1}\right] \cap\left[\theta_{A 2}, \theta_{B 2}\right]$. Since $\gamma_{A 1}^{*}\left(g_{0}\right) \in\left(\theta_{A 1}, \theta_{B 1}\right) \cap\left(\theta_{A 2}, \theta_{B 2}\right)$ and $f_{i}$ is continuous, there exists $\epsilon>0$ such that $f_{i}\left(\gamma_{A 1}^{*}\left(g_{0}\right)-\epsilon\right)>f_{i}\left(\gamma_{A 1}^{*}\left(g_{0}\right)\right)$ for all $i \in\{A, B\}$, so that proposing $\gamma_{A 1}^{*}\left(g_{0}\right)$ cannot be optimal for $A$, which is a contradiction. By a similar argument, it is impossible to have $f_{A}\left(\gamma_{A 1}^{*}\left(g_{0}\right)\right)>f_{A}\left(g_{0}\right)$ and $f_{B}\left(\gamma_{A 1}^{*}\left(g_{0}\right)\right) \geq f_{B}\left(g_{0}\right)$.

Step 3: Finally, we show that if $f_{A}\left(\gamma_{A 1}^{*}\left(g_{0}\right)\right)=f_{A}\left(g_{0}\right)$ and $f_{B}\left(\gamma_{A 1}^{*}\left(g_{0}\right)\right)=f_{B}\left(g_{0}\right)$, then $g_{0}$ must be equal to $x_{1}^{*}\left(\alpha^{*}\right)$. As shown earlier, $\gamma_{A 1}^{*}\left(g_{0}\right)=x_{1}^{*}\left(\alpha^{*}\right)$. We show that the system of equations

$$
f_{A}\left(x_{1}^{*}\left(\alpha^{*}\right)\right)=f_{A}\left(g_{0}\right) \quad f_{B}\left(x_{1}^{*}\left(\alpha^{*}\right)\right)=f_{B}\left(g_{0}\right)
$$

has a unique solution in $g_{0}$. Clearly, $g_{0}=x_{1}^{*}\left(\alpha^{*}\right)$ solves (A11). To see that no other solution exists, suppose $g^{\prime} \neq x_{1}^{*}\left(\alpha^{*}\right)$ solves (A11). Since $x_{1}^{*}\left(\alpha^{*}\right) \in\left[\theta_{A 1}, \theta_{B 1}\right] \cap\left[\theta_{A 2}, \theta_{B 2}\right]$ solves (A11) and $f_{A}$ is strictly monotone on $\left[\theta_{A 1}, \theta_{B 1}\right] \cap\left[\theta_{A 2}, \theta_{B 2}\right]$, we must have $g^{\prime} \notin\left[\theta_{A 1}, \theta_{B 1}\right] \cap\left[\theta_{A 2}, \theta_{B 2}\right]$.

Next we show that it is not possible to have $g^{\prime} \in \mathbb{R}_{+} \backslash\left[\theta_{A 2}, \theta_{B 2}\right]$ using the following lemma. 
Lemma A9. Let $Z=\{0\} \times \mathbb{R}_{+}$. Suppose $\theta_{A 2}<\theta_{B 2}$. For any $g_{1} \in \mathbb{R}_{+} \backslash\left[\theta_{A 2}, \theta_{B 2}\right], \tilde{g}\left(g_{1}\right)=$ $p_{A} \gamma_{A 2}^{*}\left(g_{1}\right)+p_{B} \gamma_{B 2}^{*}\left(g_{1}\right)$ satisfies $\tilde{g}\left(g_{1}\right) \in\left(\theta_{A 2}, \gamma_{B 2}^{*}\left(g_{1}\right)\right) \subseteq\left[\theta_{A 2}, \theta_{B 2}\right], V_{A}\left(g_{1}\right)<V_{A}\left(\tilde{g}\left(g_{1}\right)\right)$ and $V_{B}\left(g_{1}\right)<V_{B}\left(\tilde{g}\left(g_{1}\right)\right)$.

Proof. Fix $g_{1}<\theta_{A 2}$. Note that $\kappa_{i 2}^{*}\left(g_{1}\right)=0$ for all $i \in\{A, B\}$ and all $g_{1} \in \mathbb{R}_{+}$since $Z=$ $\{0\} \times \mathbb{R}_{+}$. From the proof of Proposition 4, if $g_{1}<\theta_{A 2}$, then $\gamma_{A 2}^{*}\left(g_{1}\right)=\theta_{A 2}$ and $\gamma_{B 2}^{*}\left(g_{1}\right)>\theta_{A 2}$. We also have $\gamma_{B 2}^{*}\left(g_{1}\right) \leq \theta_{B 2}$ by Proposition 4 part 1. Hence $\tilde{g}\left(g_{1}\right) \in\left(\theta_{A 2}, \gamma_{B 2}^{*}\left(g_{1}\right)\right) \subseteq\left[\theta_{A 2}, \theta_{B 2}\right]$.

Note that $V_{i}\left(g_{1}\right)=p_{A} u_{i 2}\left(\theta_{A 2}\right)+p_{B} u_{i 2}\left(\gamma_{B 2}^{*}\left(g_{1}\right)\right)$. Moreover, since $\tilde{g}\left(g_{1}\right) \in\left[\theta_{A 2}, \theta_{B 2}\right]$, we have $V_{i}\left(\tilde{g}\left(g_{1}\right)\right)=u_{i 2}\left(\tilde{g}\left(g_{1}\right)\right)=u_{i 2}\left(p_{A} \theta_{A 2}+p_{B} \gamma_{B 2}^{*}\left(g_{1}\right)\right)$ by Proposition 4 part 2. By strict concavity of $u_{i 2}$, it follows that $V_{i}\left(g_{1}\right)<V_{i}\left(\tilde{g}\left(g_{1}\right)\right)$ for $i \in\{A, B\}$. When $g_{1}>\theta_{B 2}$, the argument is similar and omitted.

If $g^{\prime} \in \mathbb{R}_{+} \backslash\left[\theta_{A 2}, \theta_{B 2}\right]$ solves (A11) we have $f_{i}\left(g^{\prime}\right)=u_{i 1}\left(g^{\prime}\right)+\delta V_{i}\left(g^{\prime}\right)=f_{i}\left(x_{1}^{*}\left(\alpha^{*}\right)\right)$ for all $i \in\{A, B\}$. From Lemma A9, there exists $\tilde{g}^{\prime} \in\left[\theta_{A 2}, \theta_{B 2}\right]$ such that $V_{i}\left(g^{\prime}\right)<V_{i}\left(\tilde{g}^{\prime}\right)$ for all $i \in\{A, B\}$. Since $\tilde{g}^{\prime} \in\left[\theta_{A 2}, \theta_{B 2}\right], V_{i}\left(\tilde{g}^{\prime}\right)=u_{i 2}\left(\tilde{g}^{\prime}\right)$ by Proposition 4 part 2 . Hence $u_{i 1}\left(g^{\prime}\right)+$ $\delta u_{i 2}\left(\tilde{g}^{\prime}\right)>f_{i}\left(x_{1}^{*}\left(\alpha^{*}\right)\right)$ for all $i \in\{A, B\}$. Furthermore, $f_{i}\left(x_{1}^{*}\left(\alpha^{*}\right)\right)=u_{i 1}\left(x_{1}^{*}\left(\alpha^{*}\right)\right)+\delta u_{i 2}\left(x_{2}^{*}\left(\alpha^{*}\right)\right)$ for all $i \in\{A, B\}$ since $x_{1}^{*}\left(\alpha^{*}\right)=x_{2}^{*}\left(\alpha^{*}\right) \in\left[\theta_{A 2}, \theta_{B 2}\right]$. Thus $u_{i 1}\left(g^{\prime}\right)+\delta u_{i 2}\left(\tilde{g}^{\prime}\right)>u_{i 1}\left(x_{1}^{*}\left(\alpha^{*}\right)\right)+$ $\delta u_{i 2}\left(x_{2}^{*}\left(\alpha^{*}\right)\right)$ for all $i \in\{A, B\}$, which violates dynamic Pareto efficiency of $\left(x_{1}^{*}\left(\alpha^{*}\right), x_{2}^{*}\left(\alpha^{*}\right)\right)$ as it is Pareto dominated by $\left(g^{\prime}, \tilde{g}^{\prime}\right)$. Hence, it is not possible to have $g^{\prime} \in \mathbb{R}_{+} \backslash\left[\theta_{A 2}, \theta_{B 2}\right]$.

Since $g^{\prime} \notin\left[\theta_{A 1}, \theta_{B 1}\right] \cap\left[\theta_{A 2}, \theta_{B 2}\right]$ and $g^{\prime} \notin \mathbb{R}_{+} \backslash\left[\theta_{A 2}, \theta_{B 2}\right]$, it follows that if $\left[\theta_{A 2}, \theta_{B 2}\right] \subseteq$ $\left[\theta_{A 1}, \theta_{B 1}\right]$, then $g_{0}=x_{1}^{*}\left(\alpha^{*}\right)$ is the unique solution to (A11). If instead $\left[\theta_{A 1}, \theta_{B 1}\right] \subseteq\left[\theta_{A 2}, \theta_{B 2}\right]$, then we need to rule out $g^{\prime} \in\left[\theta_{A 2}, \theta_{A 1}\right] \cup\left[\theta_{B 1}, \theta_{B 2}\right]$. Note that $f_{B}\left(g_{0}\right)=u_{B 1}\left(g_{0}\right)+\delta u_{B 2}\left(g_{0}\right)$ if $g_{0} \in\left[\theta_{A 2}, \theta_{B 1}\right]$ by Proposition 4 part 2 , which implies that $f_{B}\left(g_{0}\right)$ is strictly increasing in $g_{0}$ on $\left[\theta_{A 2}, \theta_{B 1}\right]$. Since $x_{1}^{*}\left(\alpha^{*}\right)$ solves (A11), it is not possible to have $g^{\prime} \in\left[\theta_{A 2}, \theta_{A 1}\right]$. By a similar argument, it is not possible to have $g^{\prime} \in\left[\theta_{B 1}, \theta_{B 2}\right]$. Thus, there is a unique solution to (A11) if $\left[\theta_{A 1}, \theta_{B 1}\right] \subseteq\left[\theta_{A 2}, \theta_{B 2}\right]$.

\section{A3.3 Proof of Proposition 6}

Denote $g_{i}^{*} \in \arg \max _{g_{1} \in \mathbb{R}_{+}} f_{i}\left(g_{1}\right)$ for $i \in\{A, B\}$ where $f_{i}$ is defined as in (A6). We first show that $g_{A}^{*}$ is unique and $g_{A}^{*} \notin\left[\theta_{A 1}, \theta_{B 1}\right]$ when $\theta_{A 2}<\theta_{A 1}<\theta_{B 2}$. The proof that $g_{B}^{*}$ is unique and $g_{B}^{*} \notin\left[\theta_{A 1}, \theta_{B 1}\right]$ when $\theta_{A 2}<\theta_{B 1}<\theta_{B 2}$ is analogous and omitted. 
Define $Q_{k} \subset \mathbb{R}_{+}$for $k=1, \ldots, 5$ as

$$
\begin{aligned}
Q_{1} & =\left(0, \max \left\{0,2 \theta_{A 2}-\theta_{B 2}\right\}\right) \\
Q_{2} & =\left(\max \left\{0,2 \theta_{A 2}-\theta_{B 2}\right\}, \theta_{A 2}\right) \\
Q_{3} & =\left(\theta_{A 2}, \theta_{B 2}\right) \\
Q_{4} & =\left(\theta_{B 2}, 2 \theta_{B 2}-\theta_{A 2}\right) \\
Q_{5} & =\left(2 \theta_{B 2}-\theta_{A 2}, \infty\right)
\end{aligned}
$$

Note that $Q_{k}$ may be empty for some $k$. Recall $V_{i}\left(g_{1}\right)$ is player $i$ 's continuation payoff when mandatory spending in period 1 is $g_{1}$. We use the following result.

Lemma A10. Let $Z=\{0\} \times \mathbb{R}_{+}$. If $u_{i t}\left(x_{t}\right)=-\left(\left|x_{t}-\theta_{i t}\right|\right)^{r}$ for all $i \in\{A, B\}$ and for all $t$ where $r>1$, then $V_{i}^{\prime \prime}\left(g_{1}\right)$ exists and $V_{i}^{\prime \prime}\left(g_{1}\right) \leq 0$ for all $i \in\{A, B\}$ whenever $g_{1} \in Q_{k}$ for some $k=1, \ldots, 5$.

Proof. First, by Proposition 4, the second period equilibrium strategies are

$$
\begin{aligned}
& \gamma_{A 2}^{*}\left(g_{1}\right)=\max \left\{\theta_{A 2}, \min \left\{g_{1}, 2 \theta_{B 2}-g_{1}\right\}\right\}, \\
& \gamma_{B 2}^{*}\left(g_{1}\right)=\min \left\{\theta_{B 2}, \max \left\{g_{1}, 2 \theta_{A 2}-g_{1}\right\}\right\} .
\end{aligned}
$$

Second, note that $\gamma_{A 2}^{*}\left(g_{1}\right)$ is constant in $g_{1}$ on $Q_{1} \cup Q_{2} \cup Q_{5}$, equal to $g_{1}$ on $Q_{3}$ and equal to $2 \theta_{B 2}-g_{1}$ on $Q_{4}$. Similarly, $\gamma_{B 2}^{*}\left(g_{1}\right)$ is constant in $g_{1}$ on $Q_{1} \cup Q_{4} \cup Q_{5}$, equal to $2 \theta_{A 2}-g_{1}$ on $Q_{2}$ and equal to $g_{1}$ on $Q_{3}$. This implies

$$
V_{i}^{\prime}\left(g_{1}\right)= \begin{cases}-p_{B} u_{i 2}^{\prime}\left(2 \theta_{A 2}-g_{1}\right) & \text { if } g_{1} \in Q_{2} \\ u_{i 2}^{\prime}\left(g_{1}\right) & \text { if } g_{1} \in Q_{3} \\ -p_{A} u_{i 2}^{\prime}\left(2 \theta_{B 2}-g_{1}\right) & \text { if } g_{1} \in Q_{4} \\ 0 & \text { if } g_{1} \in Q_{1} \cup Q_{5} .\end{cases}
$$

Thus $V_{i}^{\prime \prime}\left(g_{1}\right)$ exists and, by strict concavity of $u_{i 2}$, we have $V_{i}^{\prime \prime}\left(g_{1}\right) \leq 0$ for all $i \in\{A, B\}$ and for all $g_{1} \in Q_{k}$ for $k \in\{1, \ldots, 5\}$.

By Lemma A10, if $Z=\{0\} \times \mathbb{R}_{+}$and $u_{i t}\left(x_{t}\right)=-\left(x_{t}-\theta_{i t}\right)^{2}$ for all $i \in\{A, B\}$ and all $t$, then $V_{A}$ is continuously differentiable on $\mathbb{R}_{+} \backslash\left\{2 \theta_{A 2}-\theta_{B 2}, \theta_{A 2}, \theta_{B 2}, 2 \theta_{B 2}-\theta_{A 2}\right\}$. Inspection of (A12) shows that $V_{A}\left(g_{1}\right)$ is increasing on $\left[0, \theta_{A 2}\right]$. Since $f_{A}\left(g_{1}\right)=u_{A 1}\left(g_{1}\right)+\delta V_{A}\left(g_{1}\right)$ and $\theta_{A 1}>\theta_{A 2}$, we have $g_{A}^{*}>\theta_{A 2}$. Similarly, since $f_{A}$ is strictly decreasing on $Q_{5}$, it is not possible 
to have $g_{A}^{*} \in Q_{5}$. From $(\mathrm{A} 12)$ and $\theta_{A 2}<\theta_{B 2}$, we have

$$
\begin{gathered}
\lim _{g_{1} \rightarrow \theta_{B 2}^{-}} V_{A}^{\prime}\left(g_{1}\right)=u_{A 2}^{\prime}\left(\theta_{B 2}\right)<0<\lim _{g_{1} \rightarrow \theta_{B 2}^{+}} V_{A}^{\prime}\left(g_{1}\right)=-p_{A} u_{A 2}^{\prime}\left(\theta_{B 2}\right), \\
\lim _{g_{1} \rightarrow\left(2 \theta_{B 2}-\theta_{A 2}\right)^{-}} V_{A}^{\prime}\left(g_{1}\right)=0=\lim _{g_{1} \rightarrow\left(2 \theta_{B 2}-\theta_{A 2}\right)^{+}} V_{A}^{\prime}\left(g_{1}\right) .
\end{gathered}
$$

This implies $g_{A}^{*} \notin\left\{\theta_{B 2}, 2 \theta_{B 2}-\theta_{A 2}\right\}$. Thus $g_{A}^{*} \in Q_{3} \cup Q_{4}$ and $f_{A}^{\prime}\left(g_{A}^{*}\right)=0$.

Suppose $g_{A, k}^{*}$ satisfies $f_{A}^{\prime}\left(g_{A, k}^{*}\right)=0$ and $g_{A, k}^{*} \in Q_{k}$ for $k \in\{3,4\}$, then $g_{A, 3}^{*}=\frac{\theta_{A 1}+\delta \theta_{A 2}}{1+\delta}$ and $g_{A, 4}^{*}=\frac{\theta_{A 1}+\delta p_{A}\left(2 \theta_{B 2}-\theta_{A 2}\right)}{1+\delta p_{A}}$.

Since $\theta_{A 2}<\theta_{A 1}$, we have $g_{A, 3}^{*} \in\left(\theta_{A 2}, \theta_{A 1}\right)$ and hence $g_{A, 3}^{*} \in\left[\theta_{A 2}, \theta_{B 2}\right]$. We need to show that $g_{A, 4}^{*}$ does not maximize $f_{A}$ when $\theta_{A 2}<\theta_{A 1}<\theta_{B 2}$. By Proposition 4 part 2 we can evaluate $f_{A}$ at $g_{A, 3}^{*}$ and $g_{A, 4}^{*}$ and compare these values. We have

$$
\begin{aligned}
& f_{A}\left(g_{A, 3}^{*}\right)=-\frac{\delta}{1+\delta}\left(\theta_{A 1}-\theta_{A 2}\right)^{2}, \\
& f_{A}\left(g_{A, 4}^{*}\right)=-\frac{\delta p_{A}}{1+\delta p_{A}}\left(2 \theta_{B 2}-\theta_{A 1}-\theta_{A 2}\right)^{2}-\delta\left(1-p_{A}\right)\left(\theta_{B 2}-\theta_{A 2}\right)^{2} .
\end{aligned}
$$

Using (A14), $f_{A}\left(g_{A, 4}^{*}\right)<f_{A}\left(g_{A, 3}^{*}\right)$ is equivalent to

$$
\frac{1}{1+\delta}\left(\theta_{A 1}-\theta_{A 2}\right)^{2}<p_{A}\left(\frac{1}{1+\delta p_{A}}\right)\left(2 \theta_{B 2}-\theta_{A 1}-\theta_{A 2}\right)^{2}+\left(1-p_{A}\right)\left(\theta_{B 2}-\theta_{A 2}\right)^{2}
$$

Note that for $p_{A} \in(0,1)$ and $\delta \in(0,1]$ we have $\frac{1}{1+\delta}<\frac{1}{1+\delta p_{A}}<1$. In addition by $\theta_{A 2}<\theta_{A 1}<\theta_{B 2}$ we have $\left(\theta_{A 1}-\theta_{A 2}\right)^{2}<\left(2 \theta_{B 2}-\theta_{A 1}-\theta_{A 2}\right)^{2}$ and $\left(\theta_{A 1}-\theta_{A 2}\right)^{2}<\left(\theta_{B 2}-\theta_{A 2}\right)^{2}$. Thus the right side of (A15) is a weighted average of two values, each of which is strictly larger than the value on the left side. Hence $g_{A, 3}^{*}$ is the unique global maximum and is statically Pareto inefficient.

The following lemma completes the proof.

Lemma A11. Let $Z=\{0\} \times \mathbb{R}_{+}$. If $f_{i}$ has a unique global maximum at $g_{i}^{*}$ for some $i \in\{A, B\}$, then there exists an open interval $\mathcal{I}$ containing $g_{i}^{*}$ such that if $g_{0} \in \mathcal{I}$, then $\gamma_{j 1}^{*}\left(g_{0}\right) \in \mathcal{I}$ for all $j \in\{A, B\}$.

Proof. Fix $i \in\{A, B\}$. Note that in any equilibrium $\sigma^{*}$, we have $f_{i}\left(\gamma_{j 1}^{*}\left(g_{0}\right)\right) \geq f_{i}\left(g_{0}\right)$ for any $j \in\{A, B\}$ and any initial status quo $g_{0} \in \mathbb{R}_{+}$since party $i$ can always propose $g_{0}$ when it is the proposer and can always reject a proposal not equal to $g_{0}$ when it is the responder.

Since $g_{i}^{*}$ is the unique global maximum of $f_{i}$ and $f_{i}$ is continuous, there exists an open 
interval $\mathcal{I}$ containing $g_{i}^{*}$ such that if $g_{0} \in \mathcal{I}$ and $\hat{g}_{0} \notin \mathcal{I}$, then $f_{i}\left(g_{0}\right)>f_{i}\left(\hat{g}_{0}\right)$. It follows that if $f_{i}\left(\tilde{g}_{0}\right) \geq f_{i}\left(g_{0}\right)$ where $g_{0} \in \mathcal{I}$, then $\tilde{g}_{0} \in \mathcal{I}$.

Consider $g_{0} \in \mathcal{I}$. Suppose party $i$ is the proposer in period 1. Since $f_{i}\left(\gamma_{i 1}^{*}\left(g_{0}\right)\right) \geq f_{i}\left(g_{0}\right)$, it follows that $\gamma_{i 1}^{*}\left(g_{0}\right) \in \mathcal{I}$. Suppose party $j \neq i$ is the proposer in period 1 . Since $f_{i}\left(\gamma_{j 1}^{*}\left(g_{0}\right)\right) \geq$ $f_{i}\left(g_{0}\right)$, it follows that $\gamma_{j 1}^{*}\left(g_{0}\right) \in \mathcal{I}$.

\section{A3.4 Efficiency with mandatory spending only}

Proposition A1. Under a budgetary institution that allows only mandatory spending programs, any equilibrium $\sigma^{*}$ is dynamically Pareto efficient for any initial status quo $g_{0} \in \mathbb{R}_{+}$, if either of the following conditions holds:

1. $\theta_{A 2}=\theta_{B 2}$.

2. $u_{i 1}=u_{i 2}$ for all $i \in\{A, B\}$.

Proof. Suppose first $\theta_{A 2}=\theta_{B 2}$. By Proposition 4 part $1, \gamma_{i 2}^{*}\left(g_{1}\right)=\theta_{A 2}$ for all $i \in\{A, B\}$ and all $g_{1} \in \mathbb{R}_{+}$. Thus, the equilibrium level of spending in period 2 is statically Pareto efficient for any $g_{0}$. The continuation value of each party $i \in\{A, B\}$ is thus $V_{i}\left(g_{1}\right)=u_{i 2}\left(\theta_{A 2}\right)$ for all $g_{1} \in \mathbb{R}_{+}$. Given the initial status quo $g_{0}$, the problem of the proposing party $i \in\{A, B\}$ in the first period is $\max _{\left(0, g_{1}\right) \in Z} u_{i 1}\left(g_{1}\right)+\delta u_{i 2}\left(\theta_{A 2}\right)$ subject to $u_{j 1}\left(g_{1}\right)+\delta u_{j 2}\left(\theta_{A 2}\right) \geq u_{j 1}\left(g_{0}\right)+\delta u_{j 2}\left(\theta_{A 2}\right)$. Since $u_{i 2}\left(\theta_{A 2}\right)$ and $u_{j 2}\left(\theta_{A 2}\right)$ are constants, this problem is equivalent to (SSP) with $\bar{u}=$ $u_{j 1}\left(g_{0}\right)$. Thus, the equilibrium level of spending in period 1 is also statically Pareto efficient for any $g_{0}$. Therefore the equilibrium is dynamically Pareto efficient by Lemma A3.

Suppose now $u_{i 1}=u_{i 2}$ for all $i \in\{A, B\}$. The following lemmas are useful.

Lemma A12. Suppose $u_{i t}=u_{i t^{\prime}}$ for all $i \in\{A, B\}$ and all $t$ and $t^{\prime}$. An allocation $\mathbf{x}=\left\{x_{t}\right\}_{t=1}^{T}$ is dynamically Pareto efficient if and only if $x_{t^{\prime}}=x_{t} \in\left[\theta_{A t}, \theta_{B t}\right]$ for all $t$ and $t^{\prime}$.

Proof. Note that $u_{i t}=u_{i t^{\prime}}$ for all $i \in\{A, B\}$ and all $t$ and $t^{\prime}$ implies $\theta_{i t}=\theta_{i t^{\prime}}$ for all $i \in\{A, B\}$ and all $t$ and $t^{\prime}$.

We first prove the "only if" part. If $\mathbf{x}^{*}=\boldsymbol{\theta}_{A}$, or $\mathbf{x}^{*}=\boldsymbol{\theta}_{B}$, then the result follows immediately. Suppose $\mathbf{x}^{*} \neq \boldsymbol{\theta}_{A}$ and $\mathbf{x}^{*} \neq \boldsymbol{\theta}_{B}$. There are two cases to consider: (i) Suppose 
$\theta_{A t}=\theta_{B t}$ for some $t$. This implies $\theta_{A t}=\theta_{B t}$ for all $t$. By Proposition 2 part $1, x_{t}^{*}=\theta_{i t}$ for all $t$, and hence $x_{t}^{*}=x_{t^{\prime}}^{*}$ for all $t$ and $t^{\prime}$. (ii) Suppose $\theta_{A t} \neq \theta_{B t}$ for some $t$. This implies $\theta_{A t} \neq \theta_{B t}$ for all $t$. By Proposition 2 part 2, we have, for any $t$ and $t^{\prime}$,

$$
\frac{u_{A t}^{\prime}\left(x_{t}^{*}\right)}{u_{B t}^{\prime}\left(x_{t}^{*}\right)}=\frac{u_{A t^{\prime}}^{\prime}\left(x_{t^{\prime}}^{*}\right)}{u_{B t^{\prime}}^{\prime}\left(x_{t^{\prime}}^{*}\right)} \text {. }
$$

Since $u_{i t}=u_{i t^{\prime}}$ for all $i \in\{A, B\}$ and all $t$ and $t^{\prime}$, and the solution to $-\frac{u_{A t}^{\prime}(x)}{u_{B t}^{\prime}(x)}=\lambda^{*}$ is unique for any $\lambda^{*}>0$ and $t$ as shown in the proof of Lemma 1, it follows that $x_{t^{\prime}}^{*}=x_{t}^{*}$. By Proposition 2 part 1 , we have $x_{t}^{*} \in\left[\theta_{A t}, \theta_{B t}\right]$.

We now prove the "if" part. As shown in the previous paragraph, any dynamically Pareto efficient allocation $\mathbf{x}^{*}$ satisfies $\mathbf{x}^{*}=\{\tilde{x}\}_{t=1}^{T}$ for some $\tilde{x}$. Note that we can let $u_{i}=u_{i t}$ for any $i \in\{A, B\}$ and rewrite (DSP) as $\max _{x \in \mathbb{R}_{+}} u_{i}(x) \sum_{t=1}^{T} \delta^{t-1}$ subject to $u_{j}(x) \sum_{t=1}^{T} \delta^{t-1} \geq \bar{U}$, which is equivalent to (SSP). By Proposition 1 , if $\tilde{x} \in\left[\theta_{A 1}, \theta_{B 1}\right]$, then $\tilde{x}$ solves (SSP) for $\bar{u}=u_{j}(\tilde{x})$. It follows that $\{\tilde{x}\}_{t=1}^{T}$ solves (DSP) for $\bar{U}=u_{j}(\tilde{x}) \sum_{t=1}^{T} \delta^{t-1}$.

Lemma A13. Let $Z=\{0\} \times \mathbb{R}_{+}$. If $u_{i 1}=u_{i 2}$ for all $i \in\{A, B\}$, then for any equilibrium $\sigma^{*}$ and initial status quo $g_{0}$, we have $\gamma_{i 1}^{*}\left(g_{0}\right)=x_{2}^{*}(\bar{U})$ where $\bar{U}=f_{j}\left(g_{0}\right)$.

Proof. When both positive and negative discretionary and mandatory spending are allowed, by the argument in the proof of Lemma 4 , the period-1 proposal satisfies $\gamma_{i 1}^{*}\left(g_{0}\right)=x_{2}^{*}(\bar{U})$ and $\kappa_{i 1}^{*}\left(g_{0}\right)=x_{1}^{*}(\bar{U})-x_{2}^{*}(\bar{U})$ where $\bar{U}=f_{j}\left(g_{0}\right)$. Since $u_{i 1}=u_{i 2}$, by Lemma A12, any $\left(x_{1}^{*}(\bar{U}), x_{2}^{*}(\bar{U})\right)$ satisfies $x_{1}^{*}(\bar{U})=x_{2}^{*}(\bar{U})$, which implies $x_{1}^{*}(\bar{U})-x_{2}^{*}(\bar{U})=0$. Hence, when only mandatory spending is allowed, which requires that $\kappa_{i 1}^{*}\left(g_{0}\right)=0$, the equilibrium period-1 proposal still satisfies $\gamma_{i 1}^{*}\left(g_{0}\right)=x_{2}^{*}(\bar{U})$.

For notational convenience we omit $\bar{U}$ below. By Lemma A12, $x_{1}^{*}=x_{2}^{*} \in\left[\theta_{A 2}, \theta_{B 2}\right]$. By Lemma A13, $\gamma_{i 1}^{*}\left(g_{0}\right)=x_{2}^{*}$ for all $i \in\{A, B\}$ and any initial status quo $g_{0} \in \mathbb{R}_{+}$. It follows that $\gamma_{i 1}^{*}\left(g_{0}\right) \in\left[\theta_{A 2}, \theta_{B 2}\right]$. By Proposition 4 part 2, we have $\gamma_{A 2}^{*}\left(\gamma_{i 1}^{*}\left(g_{0}\right)\right)=\gamma_{B 2}^{*}\left(\gamma_{i 1}^{*}\left(g_{0}\right)\right)=\gamma_{i 1}^{*}\left(g_{0}\right)$. Hence, for any $\sigma^{*}$, we have $\mathbf{x}^{\sigma^{*}}\left(g_{0}\right)=\left(x_{1}^{*}, x_{2}^{*}\right)$ with $x_{1}^{*}=x_{2}^{*} \in\left[\theta_{A 2}, \theta_{B 2}\right]$ for any $g_{0} \in \mathbb{R}_{+}$. By Lemma A12, any $\sigma^{*}$ is dynamically Pareto efficient given $g_{0} \in \mathbb{R}_{+}$. 


\section{A4 State-contingent mandatory spending}

\section{A4.1 Proof of Proposition 9}

We prove part 1 by contradiction. Suppose $x_{t}^{*} \neq x_{t^{\prime}}^{*}$ for some $t \neq t^{\prime}$. Then there exists $s \in S$ such that $x_{t}^{*}(s) \neq x_{t^{\prime}}^{*}(s)$. Without loss of generality, assume $x_{t}^{*}(s)<x_{t^{\prime}}^{*}(s)$.

From strict concavity of $u_{i}$ for all $i \in\{A, B\}$, we have $\alpha u_{i}\left(x_{t}^{*}(s), s\right)+(1-\alpha) u_{i}\left(x_{t^{\prime}}^{*}(s), s\right)<$ $u_{i}\left(\alpha x_{t}^{*}(s)+(1-\alpha) x_{t^{\prime}}^{*}(s), s\right)$ for any $\alpha \in(0,1)$. Let $\alpha=\frac{\delta^{t-1}}{\delta^{t-1}+\delta^{t^{\prime}}-1} \in(0,1)$ and $x^{\prime}=\alpha x_{t}^{*}(s)+$ $(1-\alpha) x_{t^{\prime}}^{*}(s)$, we have

$$
\delta^{t-1} u_{i}\left(x_{t}^{*}(s), s\right)+\delta^{t^{\prime}-1} u_{i}\left(x_{t^{\prime}}^{*}(s), s\right)<\left(\delta^{t-1}+\delta^{t^{\prime}-1}\right) u_{i}\left(x^{\prime}, s\right)
$$

for all $i \in\{A, B\}$, which contradicts that $\mathrm{x}^{*}$ is a solution to (DSP-S).

Next we prove part 2. Fix $i, j \in\{A, B\}$ with $i \neq j$. For any $\bar{U}>\sum_{t=1}^{T} \delta^{t-1} \mathbb{E}_{s}\left[u_{j}\left(\theta_{j s}, s\right)\right]$, (DSP-S) has no solution, so assume $\bar{U} \leq \sum_{t=1}^{T} \delta^{t-1} \mathbb{E}_{s}\left[u_{j}\left(\theta_{j s}, s\right)\right]$.

For $\bar{U}=\sum_{t=1}^{T} \delta^{t-1} \mathbb{E}_{s}\left[u_{j}\left(\theta_{j s}, s\right)\right]$, the solution to (DSP-S) is $x_{t}^{*}(s)=\theta_{j s}$ for all $t$ and $s \in S$

and for any $\bar{U} \leq \sum_{t=1}^{T} \delta^{t-1} \mathbb{E}_{s}\left[u_{j}\left(\theta_{i s}, s\right)\right]$, the solution to (DSP-S) is $x_{t}^{*}(s)=\theta_{i s}$ for all $t$ and $s \in S$. What remains is the case when $\bar{U} \in\left(\sum_{t=1}^{T} \delta^{t-1} \mathbb{E}_{s}\left[u_{j}\left(\theta_{i s}, s\right)\right], \sum_{t=1}^{T} \delta^{t-1} \mathbb{E}_{s}\left[u_{j}\left(\theta_{j s}, s\right)\right]\right)$. From the Lagrangian for (DSP-S), the first order necessary condition with respect to $x_{t}(s)$ for any $t$ and $s \in S$ is $\delta^{t-1} u_{i}^{\prime}\left(x_{t}^{*}(s), s\right)+\lambda^{*} \delta^{t-1} u_{j}^{\prime}\left(x_{t}^{*}(s), s\right)=0$ for some $\lambda^{*} \in(0, \infty)$, which simplifies to $-\frac{u_{i}^{\prime}\left(x_{t}^{*}(s), s\right)}{u_{j}^{\prime}\left(x_{t}^{*}(s), s\right)}=\lambda^{*}$.

\section{A4.2 Proof of Proposition 10}

Suppose the state in period 1 is $s_{1}$. Consider the following problem:

$$
\begin{aligned}
\max _{\left\{x_{t}: S \rightarrow \mathbb{R}_{+}\right\}_{t=1}^{T}} & u_{i}\left(x_{1}\left(s_{1}\right), s_{1}\right)+\sum_{t=2}^{T} \delta^{t-1} \mathbb{E}_{s}\left[u_{i}\left(x_{t}(s), s\right)\right] \\
\text { s.t. } & u_{j}\left(x_{1}\left(s_{1}\right), s_{1}\right)+\sum_{t=2}^{T} \delta^{t-1} \mathbb{E}_{s}\left[u_{j}\left(x_{t}(s), s\right)\right] \geq \bar{U}^{\prime},
\end{aligned}
$$

for some $\bar{U}^{\prime} \in \mathbb{R}, i, j \in\{A, B\}$ and $i \neq j$.

The difference between (DSP-S') and (DSP-S) is that $x_{1}(s)$ for $s \in S \backslash\left\{s_{1}\right\}$ does not enter (DSP-S'), so the solution to (DSP-S') does not pin down $x_{1}(s)$ for $s \in S \backslash\left\{s_{1}\right\}$.

Analogous to the proof of Proposition 7, consider the following alternative way of writing 
the social planner's problem:

$$
\begin{aligned}
\max _{\left\{x_{t}^{A}, x_{t}^{B}: S \rightarrow \mathbb{R}_{+}\right\}_{t=1}^{T}} & u_{i}\left(x_{1}^{i}\left(s_{1}\right), s_{1}\right)+\sum_{t=2}^{T} \delta^{t-1} \mathbb{E}_{s}\left[p_{A} u_{i}\left(x_{t}^{A}(s), s\right)+p_{B} u_{i}\left(x_{t}^{B}(s), s\right)\right] \\
\text { s.t. } & u_{j}\left(x_{1}^{i}\left(s_{1}\right), s_{1}\right)+\sum_{t=2}^{T} \delta^{t-1} \mathbb{E}_{s}\left[p_{A} u_{j}\left(x_{t}^{A}(s), s\right)+p_{B} u_{j}\left(x_{t}^{B}(s), s\right)\right] \geq \bar{U}^{\prime} .
\end{aligned}
$$

for some $\bar{U}^{\prime} \in \mathbb{R}, i, j \in\{A, B\}$ and $i \neq j$.

Since $u_{A}$ and $u_{B}$ are strictly concave in $x$ for all $s$, clearly any solution to (DSP-S") satisfies $x_{t}^{A}(s)=x_{t}^{B}(s)$ for all $t$ and $s$. So we can just consider (DSP-S').

Lemma A14. If $\mathbf{x}$ is a solution to (DSP-S'), then for any $t, t^{\prime} \geq 2, x_{t}=x_{t^{\prime}}$. Moreover, $x_{1}\left(s_{1}\right)=x_{t}\left(s_{1}\right)$ for $t \geq 2$.

The proof of Lemma A14 is immediate from the proof of Proposition 9. We then have the following result.

Lemma A15. If $\mathbf{x}$ is a solution to (DSP-S) for some $\bar{U}$, then it is a solution to (DSP-S') for some $\bar{U}^{\prime}$. If $\mathbf{x}$ is a solution to (DSP-S') for some $\bar{U}^{\prime}$ and it satisfies that $x_{1}(s)=x_{t}(s)$ for $t \geq 2$ and for all $s$, then $\mathbf{x}$ is a solution to (DSP-S) for some $\bar{U}$.

Proof. Fix $i, j \in\{A, B\}$ with $i \neq j$ and $s_{1} \in S$ and let $p_{1}$ be the probability distribution of $s$ in period 1. First, note that $x_{1}(s)$ for any $s \in S \backslash\left\{s_{1}\right\}$ does not enter either the objective function or the constraint in (DSP-S'). Hence if $\mathbf{x}$ is a solution to (DSP-S) with $\bar{U}$, then $\mathbf{x}$ is a solution to (DSP-S') with $\bar{U}^{\prime}=\bar{U}+\left(1-p_{1}\left(s_{1}\right)\right) u_{j}\left(x_{1}\left(s_{1}\right), s_{1}\right)-\sum_{s \in S \backslash\left\{s_{1}\right\}} p_{1}(s) u_{j}\left(x_{1}(s), s\right)$. Second, note that by Proposition 9, if $\mathbf{x}=\left\{x_{t}\right\}_{t=1}^{T}$ is a solution to (DSP-S), then $x_{t}=x_{t^{\prime}}$ for any $t$ and $t^{\prime}$. Hence, if $\mathbf{x}$ with $x_{1}(s)=x_{t}(s)$ for $t \geq 2$ and for all $s \in S$ solves (DSP-S') with $\bar{U}^{\prime}$, then $\mathbf{x}$ is a solution to (DSP-S) with $\bar{U}=\bar{U}^{\prime}-\left(1-p_{1}\left(s_{1}\right)\right) u_{j}\left(x_{1}\left(s_{1}\right), s_{1}\right)+\sum_{s \in S \backslash\left\{s_{1}\right\}} p_{1}(s) u_{j}\left(x_{1}(s), s\right)$.

We prove Proposition 10 by establishing Lemmas A16 and A17 below. With slight abuse of terminology, we call a spending rule $g \in \mathcal{M}$ dynamically Pareto efficient if $\left\{g_{t}\right\}_{t=1}^{T}$ with $g_{t}=g$ for all $t$ is a dynamically Pareto efficient allocation rule.

Lemma A16. For any $t$, if the status quo $g_{t-1}$ is dynamically Pareto efficient, then $\gamma_{i t}\left(g_{t-1}, s_{t}\right)=$ $g_{t-1}$ for all $s_{t} \in S$ and all $i \in\{A, B\}$. 
Proof. Suppose the state in period $t$ is $s_{t}$. For any status quo $g_{t-1}$ in period $t$, the proposer $i$ 's equilibrium continuation payoff is weakly higher than $u_{i}\left(g_{t-1}\left(s_{t}\right), s_{t}\right)+\sum_{t^{\prime}=t+1}^{T} \delta^{t^{\prime}-t} \mathbb{E}_{s}\left[u_{i}\left(g_{t-1}(s), s\right)\right]$ and the responder $j$ 's equilibrium continuation payoff is weakly higher than $u_{j}\left(g_{t-1}\left(s_{t}\right), s_{t}\right)+$ $\sum_{t^{\prime}=t+1}^{T} \delta^{t^{\prime}-t} \mathbb{E}_{s}\left[u_{j}\left(g_{t-1}(s), s\right)\right]$. To see this, note that for any status quo in any period, a responder accepts a proposal if it is the same as the status quo, implying that a proposer can maintain the status quo by proposing it. Hence, proposer $i$ can achieve the payoff above by proposing to maintain the status quo in period $t$ and in future periods continue to propose to maintain the status quo if it is the proposer and rejects any proposal other than the status quo if it is the responder. Similarly, responder $j$ can achieve the payoff above by rejecting any proposal other than the status quo in period $t$ and in future periods continue to reject any proposal other than the status quo if it is the responder and propose to maintain the status quo if it is the proposer.

Consider proposer $i$ 's problem in period $t$

$$
\begin{aligned}
\max _{g_{t} \in \mathcal{M}} & u_{i}\left(g_{t}\left(s_{t}\right), s_{t}\right)+\delta V_{i t}\left(g_{t} ; \sigma^{*}\right) \\
\text { s.t. } & u_{j}\left(g_{t}\left(s_{t}\right), s_{t}\right)+\delta V_{j t}\left(g_{t} ; \sigma^{*}\right) \geq u_{j}\left(g_{t-1}\left(s_{t}\right), s_{t}\right)+\delta V_{j t}\left(g_{t-1} ; \sigma^{*}\right),
\end{aligned}
$$

where $V_{i t}\left(g ; \sigma^{*}\right)$ is the expected discounted utility of party $i \in\{A, B\}$ in period $t$ generated by strategies $\sigma^{*}$ when the status quo is $g$. As shown in the previous paragraph, $u_{j}\left(g_{t-1}\left(s_{t}\right), s_{t}\right)+$ $\left.\delta V_{j t}\left(g_{t-1}, \sigma^{*}\right) \geq u_{j}\left(g_{t-1}\left(s_{t}\right), s_{t}\right)+\sum_{t^{\prime}=t+1}^{T} \delta^{t^{\prime}-t} \mathbb{E}_{s}\left[u_{j}\left(g_{t-1}(s), s\right)\right)\right]$.

Suppose the solution to the proposer's problem in period $t$ is $g_{t}^{*} \neq g_{t-1}$. Then there exists an allocation with $x_{t}=g_{t}^{*}$ and future allocations induced by status quo $g_{t}^{*}$ and $\sigma^{*}$ such that party $i$ 's dynamic payoff is higher than $u_{i}\left(g_{t-1}\left(s_{t}\right), s_{t}\right)+\sum_{t^{\prime}=t+1}^{T} \delta^{t^{\prime}-t} \mathbb{E}_{s}\left[u_{i}\left(g_{t-1}(s), s\right)\right]$ and party $j$ 's dynamic payoff is higher than $\left.u_{j}\left(g_{t-1}\left(s_{t}\right), s_{t}\right)+\sum_{t^{\prime}=t+1}^{T} \delta^{t^{\prime}-t} \mathbb{E}_{s}\left[u_{j}\left(g_{t-1}(s), s\right)\right)\right]$. But if $g_{t-1}$ is dynamically Pareto efficient, then having allocation in all periods $t^{\prime} \geq t$ equal to $g_{t-1}$ is a solution to (DSP-S') with $\left.\bar{U}=u_{j}\left(g_{t-1}\left(s_{t}\right), s_{t}\right)+\sum_{t^{\prime}=t+1}^{T} \delta^{t^{\prime}-t} \mathbb{E}_{s}\left[u_{j}\left(g_{t-1}(s), s\right)\right)\right]$, a contradiction.

Lemma A17. For any initial status quo $g_{0}$ and any $s_{1} \in S$, the proposer makes a proposal in period 1 that is dynamically Pareto efficient, that is, $\gamma_{i 1}\left(g_{0}, s_{1}\right)$ is dynamically Pareto efficient for all $i \in\{A, B\}$. 
Proof. Fix $g_{0}$ and $s_{1}$. Let $f_{j}\left(g_{0}, s_{1}\right)$ be the responder $j$ 's status quo payoff. That is,

$$
f_{j}\left(g_{0}, s_{1}\right)=u_{j}\left(g_{0}\left(s_{1}\right), s_{1}\right)+\delta V_{j 1}\left(g_{0} ; \sigma^{*}\right) .
$$

Let $\bar{U}^{\prime}=f_{j}\left(g_{0}, s_{1}\right)$ and denote the solution to (DSP-S') by $\mathbf{x}\left(\bar{U}^{\prime}\right)=\left(x_{1}\left(\bar{U}^{\prime}\right), \ldots, x_{T}\left(\bar{U}^{\prime}\right)\right)$. By Lemma A14, $x_{t}\left(\bar{U}^{\prime}\right)=x_{t^{\prime}}\left(\bar{U}^{\prime}\right)$ for any $t, t^{\prime} \geq 2$. Without loss of generality, suppose $\mathbf{x}\left(\bar{U}^{\prime}\right)$ satisfies $x_{1}\left(\bar{U}^{\prime}\right)=x_{t}\left(\bar{U}^{\prime}\right)$ for $t \geq 2$. Note that $x_{1}\left(\bar{U}^{\prime}\right)$ is a dynamically Pareto efficient allocation.

We next show that $\gamma_{i 1}^{*}\left(g_{0}, s_{1}\right)=x_{1}\left(\bar{U}^{\prime}\right)$. First note that if $\gamma_{i 1}^{*}\left(g_{0}, s_{1}\right)=x_{1}\left(\bar{U}^{\prime}\right)$, then, since $x_{1}\left(\bar{U}^{\prime}\right)$ is dynamically Pareto efficient, the induced equilibrium allocation is $\mathbf{x}\left(\bar{U}^{\prime}\right)$ by Lemma A16. We show by contradiction that $\gamma_{i 1}^{*}\left(g_{0}, s_{1}\right)=x_{1}\left(\bar{U}^{\prime}\right)$ is the solution to the proposer's problem. Suppose not. Then proposing $\gamma_{i 1}^{*}\left(g_{0}, s_{1}\right)$ is strictly better than proposing $x_{1}\left(\bar{U}^{\prime}\right)$, that is, proposing $\gamma_{i 1}^{*}\left(g_{0}, s_{1}\right)$ gives $i$ a strictly higher dynamic payoff while giving $j$ a dynamic payoff at least as high as $f_{j}\left(g_{0}, s_{1}\right)$. But since $\mathbf{x}\left(\bar{U}^{\prime}\right)$ is a solution to (DSP-S') and hence a solution to (DSP-S"), this is a contradiction. 